\title{
Forma Assintótica de Soluções Periódicas de uma Equação Diferencial no Plano com Retardamento
}

\author{
Mara Sueli Simão Moraes
}

Orientador: Prof. Dr. Plácido Zoega Táboas

Tese apresentada ao Instituto de Ciências Matemáticas de São Carlos, Universidade de São Paulo, para obtenção do

Título de Doutor em Ciências - Área: Matemática - Análise

São Carlos - S.P.

1994 
Aos Meus Pais Ao Saulo e Ao Bruno 


\section{AGRADECIMENTOS}

Ao Prof. Dr. Plácido Zoega Táboas, pela seriedade profissional, dedicação e paciência com que conduziu a orientação deste trabalho.

À Profạ Drạ Sandra M. Semensato de Godoy, pela solicitude e prontidão com que sempre nos ouviu e deu sugestões.

Ao Prof. Dr. Clodoaldo G. Ragazzo, pela colaboração para a realização das simulações.

Aos colegas do Departamento de Matemática da UNESP-Bauru, pelo apoio e incentivo.

Aos colegas da pós-graduação, como também, aos funcionários e professores do ICMSC-USP, pela gentileza e carinho com que nos acolheram nestes anos todos.

Enfim, agradeço a todos aqueles que direta, ou indiretamente, colaboraram para a realização deste trabalho. 


\begin{abstract}
The singularly perturbed differential-delay equation

$$
\varepsilon \dot{x}(t)=-x(t)+F(x(t-1))
$$

is studied, with $\varepsilon>0, x=\left(x_{1}, x_{2}\right), F=\left(f_{1}, f_{2}\right), f_{1}, f_{2}: \mathbb{R} \rightarrow \mathbb{R}$ odd and differentiable up to order two at the origin.

For small $\varepsilon$ and $f=-f_{1}=f_{2}$ monotone in some interval $[-A, A], A>0$, the slowly spiralling periodic solutions $x(t)$ of the equation (1) are proved to have a square-wave shape, and are related to periodic points of the mapping $F=\left(f_{1}, f_{2}\right)$.

As it is pointed out in [1], for the scalar case, when $f$ is not monotone the convergence of $x(t)$ to the square-wave typically is not uniform, and a phenomenon similar to the Gibbs one of classical Fourier serie, must occur.
\end{abstract}




\section{Resumo}

A equação diferencial com retardamento perturbada singularmente

$$
\varepsilon \dot{x}(t)=-x(t)+F(x(t-1))
$$

é estudada, $\operatorname{com} \varepsilon>0, x=\left(x_{1}, x_{2}\right), F=\left(f_{1}, f_{2}\right), f_{1}, f_{2}: \mathbb{R} \rightarrow \mathbb{R}$, diferenciáveis até ordem 2 na origem e ímpares.

Para $\varepsilon$ pequeno e $f=-f_{1}=f_{2}$ monótona num intervalo $[-A, A], A>0$, é provado que a solução periódica lentamente espiralante $x(t)$ da equação $(1)$ tem a forma de uma "onda quadrada", e está relacionada aos pontos periódicos da função $F=\left(f_{1}, f_{2}\right)$.

Como é destacado em [1], para o caso escalar, quando $f$ não é monótona a convergência de $x(t)$ para a "onda quadrada" é tipicamente não uniforme, e ocorre um fenômeno similar ao de Gibbs, da clássica série de Fourier. 


\section{Conteúdo}

Introdução

1 Existência de um contínuo global de soluções periódicas

6

2 Estimativas assintóticas para o período quando $\varepsilon \rightarrow 0_{+}$

3 Equações de camada de transição

4 Forma assintótica das soluções periódicas quando $\varepsilon \rightarrow 0_{+}$

Observações finais

Bibliografia 


\section{Introdução}

Consideremos o sistema de equações diferenciais com retardamento autônomas

$$
\left\{\begin{array}{l}
\varepsilon \dot{x}_{1}(t)=-x_{1}(t)+f_{1}\left(x_{2}(t-1)\right) \\
\varepsilon \dot{x}_{2}(t)=-x_{2}(t)+f_{2}\left(x_{1}(t-1)\right)
\end{array}\right.
$$

onde $\varepsilon>0, x=\left(x_{1}, x_{2}\right), F=\left(f_{1}, f_{2}\right), f_{1}, f_{2}: \mathbb{R} \rightarrow \mathbb{R}, f_{1}, f_{2}$ são diferenciáveis até ordem 2 na origem e ímpares.

O objetivo deste trabalho é estudar o comportamento assintótico de certas soluções periódicas não triviais de $(1)_{\varepsilon}$, quando $\varepsilon \rightarrow 0_{+}$.

Observemos que (1) $\varepsilon$ é justamente o sistema

$$
\left\{\begin{array}{l}
\dot{x}_{1}(t)=-\alpha x_{1}(t)+\alpha f_{1}\left(x_{2}(t-1)\right) \\
\dot{x}_{2}(t)=-\alpha x_{2}(t)+\alpha f_{2}\left(x_{1}(t-1)\right)
\end{array}\right.
$$

$\operatorname{com} \varepsilon=\alpha^{-1}$

Através de uma mudança na escala do tempo o sistema é equivalente à:

$$
\left\{\begin{array}{l}
\dot{y}_{1}(t)=-y_{1}(t)+f_{1}\left(y_{2}(t-\alpha)\right) \\
\dot{y}_{2}(t)=-y_{2}(t)+f_{2}\left(y_{1}(t-\alpha)\right)
\end{array}\right.
$$

Portanto, estudar o comportamento assintótico das soluções periódicas quando $\varepsilon \rightarrow 0_{+}$de $(1)_{\varepsilon}$ é equivalente ao estudo do caso para grandes retardamentos, isto é, $\alpha \rightarrow \infty$, no sistema acima. 
Em [1], Mallet-Paret e Nussbaum tratam o problema no caso escalar,

$$
\dot{x}(t)=-\alpha x(t)+\alpha f(x(t-1))
$$

onde $x \in \mathbb{R}, f: \mathbb{R} \rightarrow \mathbb{R}$.

Nosso problema é determinar como a dinâmica do sistema $(1)_{\varepsilon}$, quanto $\varepsilon$ é pequeno, se relaciona com a dinâmica do sistema limite, para $\varepsilon \rightarrow 0_{+}$

$$
\left\{\begin{array}{l}
x_{1}(t)=f_{1}\left(x_{2}(t-1)\right) \\
x_{2}(t)=f_{2}\left(x_{1}(t-1)\right)
\end{array}\right.
$$

Exemplificando, suponhamos $f: \mathbb{R} \rightarrow \mathbb{R}$ e $a \in \mathbb{R}$, tal que, $f(-a)=-f(a)$ e $f(a)=a$.

Definamos a função $x_{4}(t)=\left(x_{1}^{4}(t), x_{2}^{4}(t)\right)$ por:

$$
\begin{array}{llrl}
x_{4}(t)=(a, a) & \text { se } & -1 \leq t<0, \\
x_{4}(t)=(a,-a) & \text { se } & 0 \leq t<1, \\
x_{4}(t)=(-a,-a) & \text { se } & 1 \leq t<2, \\
x_{4}(t)=(-a, a) & \text { se } & 2 \leq t<3 \quad \text { e }
\end{array}
$$

$x_{4}(t+4)=x_{4}(t), \quad t \in \mathbb{R}$.

Então, $x_{4}(t)$ é uma solução de período 4 de (3), com $f_{1}=f$ e $f_{2}=-f$.

Veja a Figura 1.

Uma pergunta natural é se, para $\varepsilon>0$ pequeno, o sistema $(1)_{\varepsilon}$ tem soluções periódicas $x_{\varepsilon}(t)=\left(x_{1}^{\varepsilon}(t), x_{2}^{\varepsilon}(t)\right)$ com período próximo de 4 , tais que, $x_{\varepsilon}(t)$ se aproxima da solução $x_{4}(t)$ de $(3)$, em algum sentido, quando $\varepsilon$ se aproxima de zero. Esse é o problema que vamos considerar. 


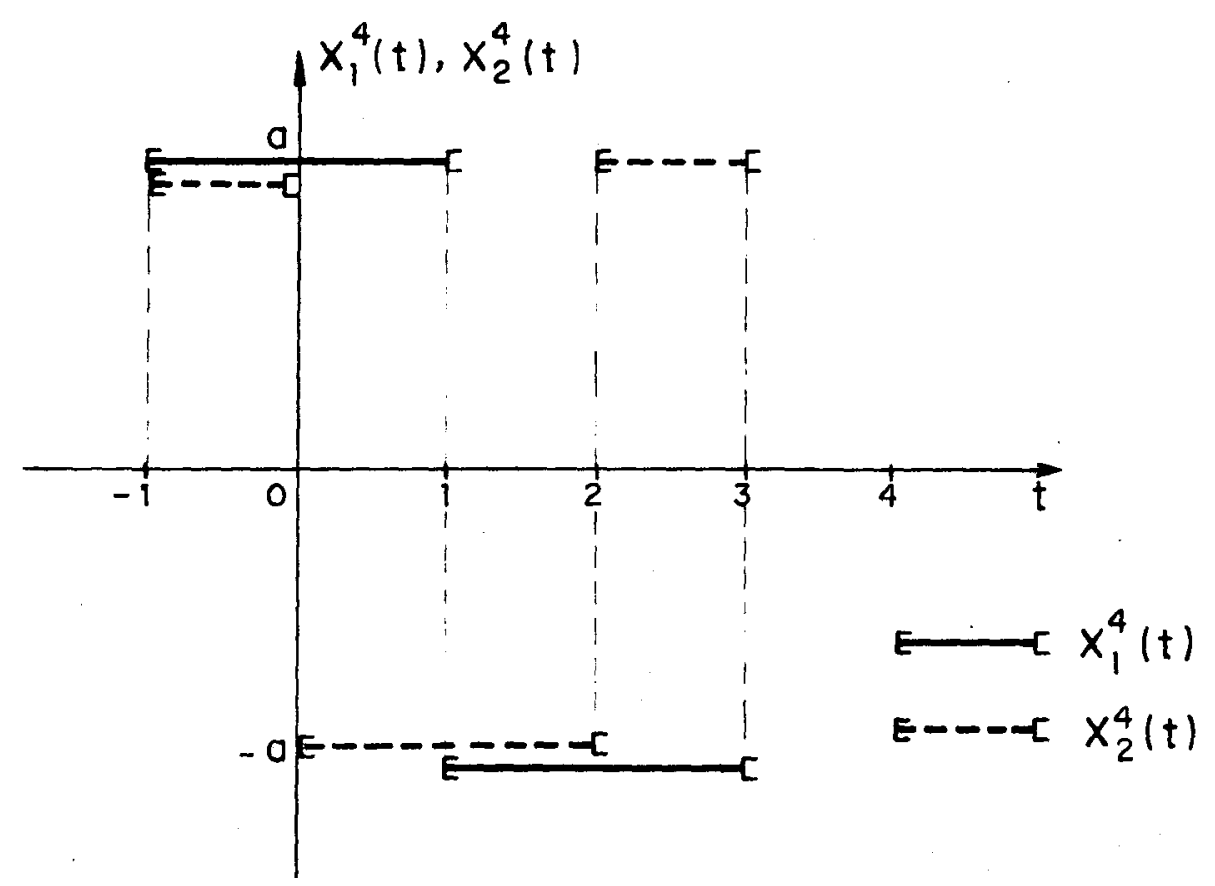

Figura 1: Uma solução de (3), com $f_{1}=f$ e $f_{2}=-f$.

Para isso, iremos seguir os seguintes passos:

(I) - Verificar a existência de um contínuo ilimitado de soluções periódicas de (1) $\varepsilon$ que emanam a partir de uma bifurcação de Hopf.

(II) - Determinar uma estimativa do período dessas soluções quando $\varepsilon \rightarrow 0_{+}$.

(III) - Estudar o comportamento assintótico das soluções de (1) $\varepsilon$ quando $\varepsilon \rightarrow 0_{+}$. Vamos mostrar que $x_{\varepsilon} \rightarrow x_{4}$ quando $\varepsilon \rightarrow 0_{+}$, uniformemente nas partes compactas de $\mathbb{R}-\mathbb{Z}$.

(IV) - Através de uma mudança de variável determinar certas equações denominadas equações camada de transição, úteis no estudo do comportamento das soluções de (1) $\varepsilon$ nos pontos de descontinuidade das soluções de (3). 
Suponhamos as seguintes hipóteses:

(H.1) $x f_{1}(x)>0, x \neq 0$,

$x f_{2}(x)<0, x \neq 0, x \in \mathbb{R}$ e $\delta=\sqrt{-\delta_{1} \delta_{2}}>1$,

onde $\delta_{1}=f_{1}^{\prime}(0), \delta_{2}=f_{2}^{\prime}(0)$.

(H.2) $\exists M>0 ;\left|f_{1}(x)\right|,\left|f_{2}(x)\right| \leq M, x \in \mathbb{R}$.

(H.3) $x \cdot F(x)<|x|^{2}$ se $x \in \bar{B}_{M}-\{0\}$,

onde "." denota o produto escalar usual do $\mathbb{R}^{2}$ e " $\bar{B}_{M}$ " denota a bola fechada de centro na origem e raio $M$ do $\mathbb{R}^{2}$.

(H.4) $\left|\delta_{1}+\delta_{2}\right|<2$.

Sendo $f_{1}, f_{2}$ diferenciáveis até ordem 2 na origem, $f_{1}$ é monótona crescente e $f_{2}$ é monótona decrescente, numa vizinhança de $x=0$. E da limitação de $f_{1}$ e $f_{2}$, segue-se do Lema 2.2 de [2] e sua demonstração, que se $x(t)$ é solução periódica $\operatorname{de}(1) \varepsilon, x(t)=\left(x_{1}(t), x_{2}(t)\right)$, então

$$
\left|x_{1}(t)\right|,\left|x_{2}(t)\right| \leq M, \text { para todo } t \in \mathbb{R}
$$

Denotaremos por $C=C\left([-1,0], \mathbb{R}^{2}\right)$ o espaço de Banach definido por

$$
C=C\left([-1,0], \mathbb{R}^{2}\right)=\left\{\varphi:[-1,0] \rightarrow \mathbb{R}^{2} ; \varphi \text { é contínua }\right\}
$$

munido da norma

$$
\|\varphi\|=\sup _{-1 \leq \theta \leq 0}|\varphi(\theta)|
$$

onde $|\cdot|$ denota a norma usual de $\mathbb{R}^{2}$.

Dados $t_{0} \geq 0, A \geq 0$ e uma função $x \in C\left(\left[t_{0}-1, t_{0}+A\right], \mathbb{R}^{2}\right)$, para cada $t \in\left[t_{0}, t_{0}+A\right]$, denotaremos por $x_{t} \in C$ a função definida por

$$
x_{t}(\theta)=x(t+\theta), \quad-1 \leq \theta \leq 0 .
$$


Se $\phi \in C$, uma solução de $(1)_{\varepsilon}$ que coincida com $\phi$ em $[-1,0]$ deve satisfazer:

$$
\begin{aligned}
& x(t)=\phi(t), \quad t \in[-1,0] \\
& x(t)=e^{-\alpha t} \phi(0)+\alpha \int_{0}^{t} e^{-\alpha(t-s)} F(x(s-1)) d s, \quad t \geq 0
\end{aligned}
$$

e reciprocamente. Por um processo recursivo em intervalos de comprimento um, a partir dessa expressão, mostra-se a existência e unicidade da solução em $[-1, \infty]$. Veja [4], para maiores informações sobre a teoria geral dessas equações. 


\section{Capítulo 1}

\section{Existência de um contínuo global de soluções periódicas}

Consideremos os seguintes subconjuntos convexos e fechados de $C$ :

$$
\begin{aligned}
& K=\left\{\varphi \in C \mid \varphi_{1}(-1) \doteq 0, \varphi_{1}, \varphi_{2} \geq 0\right\} \\
& K_{\alpha}=\left\{\varphi \in K \mid e^{\alpha \theta} \varphi_{j}(\theta) \text { monótona crescente em }[-1.0], j=1,2\right\}, \text { com }
\end{aligned}
$$
$\alpha>0$.

Ainda, seja $\alpha_{0}$ o primeiro valor onde ocorre uma bifurcação de Hopf de (2) $\alpha$ :

$$
\alpha_{0}:=\frac{b_{0}}{\sqrt{\delta^{2}-1}}, \quad 0<b_{0}:=\operatorname{arcsen} \frac{1}{\delta}<\frac{\pi}{2} .
$$

Chamamos de $x(\cdot ; \alpha, \varphi)$ a solução de $(2)_{\alpha}$ definida em $[-1, \infty)$, a qual coincide $\operatorname{com} \varphi \operatorname{em}[-1,0], \varphi \in C=C\left([-1,0], \mathbb{R}^{2}\right)$.

Em [2] , M.Z. Baptistini, estudando um caso mais geral de (2) $\alpha$ prova, sob as hipóteses (H.1) e (H.2), o seguinte teorema:

Teorema 1.1 Suponhamos válidas as hipóteses (H.1) e (H.2) e seja $\alpha_{0}$ como em (1.1). Então para todo $\alpha>\alpha_{0},(2)_{\alpha}$ tem solução periódica não trivial $x(\cdot ; \alpha, \varphi)=$ $\left(x_{1}(\cdot ; \alpha, \varphi), x_{2}(\cdot ; \alpha, \varphi)\right), \varphi \in K_{\alpha}$, com periodo $T>4$. 
A prova desse resultado segue da existência de um ponto fixo não trivial de um operador de retorno $A_{\alpha}$ definido pelo fluxo no conjunto $K_{\alpha}$ e é consequência de um resultado devido a Nussbaum [5], que usa o conceito de ejetividade de acordo com Browder [6].

Mais precisamente é mostrada a existência de uma aplicação contínua

$$
\tau_{\alpha}: C \rightarrow[4, \infty),
$$

tal que, se $A_{\alpha}: K_{\alpha} \rightarrow C$ é o operador definido por

$$
\begin{aligned}
& A_{\alpha}(\varphi)=x_{\tau_{\alpha}(\varphi)}(\cdot ; \alpha, \varphi), \varphi \neq 0 \\
& A_{\alpha}(0)=0
\end{aligned}
$$

então $A_{\alpha}$ é completamente contínuo, $A_{\alpha} K_{\alpha} \subset K_{\alpha}$ e possui um ponto fixo $\varphi \in K_{\alpha}-\{0\}$, isto é , $A_{\alpha} \varphi=\varphi$, para $\alpha>\alpha_{0}$.

O conceito de solução periódica lentamente espiralante de acordo com M.Z. Baptistini [2] é dado a seguir.

Definição 1.1 Uma solução $x(t)=\left(x_{1}(t), x_{2}(t)\right)$ de $(2)_{\alpha}$ é chamada solucão periódica lentamente espiralante se $\exists q_{1}, q_{2}, q_{3}, q_{4}>0, q_{j}>q_{j-1}+1$ para $j=2,3,4$, tais que

(i) $x_{1}(-1)=0, x_{1}(t)>0$ se $-1<t<q_{2}$ e $x_{1}(t)<0$ se $q_{2}<t<q_{4}$,

(ii) $x_{2}(t)>0$ se $-1<t<q_{1}$ e se $q_{3}<t<q_{4}, x_{2}(t)<0$ se $q_{1}<t<q_{3}$, e

(iii) $x(t)=x\left(t+q_{4}+1\right)$, para todo $t$.

Sob as hipóteses (H.1) e (H.2), M.Z. Baptistini em [2], mostra que há uma correspondência biunívoca entre soluçôes periódicas lentamente espiralantes de (2) $\alpha$ e pontos fixos nâo triviais de $A_{\alpha}$.

Como $f_{1}, f_{2}$ são funções ímpares é natural estudarmos soluções periódicas lentamente espiralantes simétricas, isto é, que satisfaçam a seguinte condição:

$$
x(t+p)=-x(t), \text { para todo } t,
$$


onde $p$ é a metade do período mínimo de $x(t)=\left(x_{1}(t), x_{2}(t)\right)$.

De fato, definamos $\tau_{\alpha}^{*}>0$, tal que, $x_{\tau_{\alpha}^{*}}(\cdot ; \alpha, \varphi) \in-K_{\alpha}$. Consideremos $x(t)=x(t ; \alpha, \varphi)$ uma solução periódica lentamente espiralante de $(2)_{\alpha}$, tal que, $x_{\tau_{\alpha}^{*}}(\cdot ; \alpha, \varphi)=-\varphi$. Seja $y(t)=-x(t)$. Então $y(t)$ é solução de $(2)_{\alpha}$, para todo $t$, e do fato de $(2)_{\alpha}$ ser autônomo, $y\left(t+\tau_{\alpha}^{*}\right)$ também é solução. Ainda $y\left(\theta+\tau_{\alpha}^{*}\right)=\varphi(\theta)$, para $-1 \leq \theta \leq 0$. Portanto, $x(t)=-x\left(t+\tau_{\alpha}^{*}\right), p=\tau_{\alpha}^{*}$ e condição (1.2) é verificada.

Seguindo Saupe [5], [6], definamos uma $S^{*}$-solução a seguir.

Definição 1.2 Uma solução $x(t)=\left(x_{1}(t), x_{2}(t)\right)$ de $(2)_{\alpha}$ é chamada de uma $S^{*}$-solução se é uma solução periódica lentamente espiralante satisfazendo (1.2).

Inspirados em [2] e devido à simetria das $S^{*}$-soluções, temos a existência da aplicação contínua

$$
\tau_{\alpha}^{*}: C \rightarrow[2, \infty)
$$

tal que, se $S_{\alpha}^{*}: K_{\alpha} \rightarrow C$ é o operador definido por

$$
\begin{aligned}
S_{\alpha}^{*}(\varphi) & =-x_{\tau_{\alpha}^{*}(\varphi)}(\cdot ; \alpha, \varphi), \varphi \neq 0 \\
S_{\alpha}^{*}(0) & =0
\end{aligned}
$$

então $S_{\alpha}^{*}$ é completamente contínuo, $S_{\alpha}^{*} K_{\alpha} \subset K_{\alpha}$.

Analogamente ao estudo feito por M.Z. Baptistini em [2], $S_{\alpha}^{*}$ tem um ponto fixo não nulo e portanto (2) $\alpha$ possui uma $S^{*}$-solução para cada $\alpha>\alpha_{0}$.

Seja:

$$
\mathcal{S}=\left\{(\alpha, \varphi) \in(0, \infty) \times K \mid \varphi \in K_{\alpha}-\{0\}, S_{\alpha}^{*}(\varphi)=\varphi\right\}
$$

e consideremos, em adição, as hipóteses (H.3) e (H.4).

Pequenas adaptações das demonstrações de M.Z. Baptistini em [2], levam ao seguinte resultado.

Teorema 1.2 Sejam $\mathcal{S}$ definido como (1.3) e $\alpha_{0}$ como em (1.1). Então valem as afirmações 
(i) $\exists \bar{\alpha}>0$ tal que se $(\alpha, \varphi) \in \mathcal{S}$ entâa $\alpha \geq \bar{\alpha}$ e $\|\varphi\| \leq M$.

(ii) $O$ fecho $\overline{\mathcal{S}}$ de $\mathcal{S} e m(0, \infty) \times K$ é $\overline{\mathcal{S}}=\mathcal{S} \cup\left\{\left(\alpha_{0}, 0\right)\right\}$.

(iii) Seja $\mathcal{S}_{0}$ a componente conexa de $\overline{\mathcal{S}}$ contendo $\left(\alpha_{0}, 0\right)$. Então $\mathcal{S}_{0}$ é um subconjunto ilimitado de $(0, \infty) \times K$, mais especificamente, para cada $\alpha>\alpha_{0}$, existe $S^{*}$-soluçâo $x(t ; \alpha, \varphi)$ de $(2)_{\alpha}$ tal que $(\alpha, \varphi) \in \mathcal{S}_{0}$ e $|x(t ; \alpha, \varphi)| \leq M$ para todo $t$. 


\section{Capítulo 2}

\section{Estimativas assintóticas para o período quando $\varepsilon \rightarrow 0_{+}$}

Consideremos o sistema de equações diferenciais com retardamento autônomas:

$$
\left\{\begin{array}{l}
\varepsilon \dot{x}_{1}(t)=-x_{1}(t)+f_{1}\left(x_{2}(t-1)\right) \\
\varepsilon \dot{x}_{2}(t)=-x_{2}(t)+f_{2}\left(x_{1}(t-1)\right), \quad \varepsilon>0
\end{array}\right.
$$

Neste capítulo vamos iniciar o estudo do comportamento assintótico das $S^{*}$ soluções, $x=\left(x_{1}, x_{2}\right)$, de $(2.1) \varepsilon$, onde:

$$
F=\left(f_{1}, f_{2}\right), f_{1}, f_{2}: \mathbb{R} \rightarrow R, \text { diferenciáveis até ordem } 2 \text { na origem e ímpares. }
$$

Iniciaremos o capítulo com um lema técnico sobre as $S^{*}$-soluções, $x=\left(x_{1}, x_{2}\right)$, $\operatorname{de}(2.1)_{\varepsilon}$

Lema 2.1 Seja $x(t)=\left(x_{1}(t), x_{2}(t)\right)$ uma $S^{*}$-solução do sistema $(2.1) \varepsilon$, $F=\left(f_{1}, f_{2}\right), f_{1}, f_{2}: \mathbb{R} \rightarrow \mathbb{R}$, diferenciáveis até ordem 2 na origem, funções impares, satisfazendo as hipóteses (H.1) e (H.2). Então os pontos extremos da coordenada $x_{2}(t)$ pertencem ao intervalo $I=\left(q_{j}, q_{j}+1\right), j=2,4$. Um resultado análogo é válido para $x_{1}(t)$. 
Prova: Seja $t_{2} \in\left[q_{1}, q_{3}\right]$.

Afirmamos que se $t_{2}$ é ponto extremo de $x_{2}(t)$, então $t_{2} \in\left(q_{2}, q_{2}+1\right)$.

Suponhamos, por contradição, que $t_{2} \geq q_{2}+1$.

Da segunda equação de $(2.1)_{\varepsilon}$ e da definição de soluções periódicas lentamente espiralantes, segue que $\dot{x}_{2}\left(t_{2}\right)>0$, o que contradiz o fato de $t_{2}$ ser ponto extremo de $x_{2}(t)$.

Agora, suponhamos, por contradição, que $t_{2} \leq q_{2}$ e consideremos

$$
\left(e^{\alpha t} x_{2}(t)\right)^{\prime}=\alpha e^{\alpha t} f_{2}\left(x_{1}(t-1)\right), \quad t \in\left(q_{1}, q_{2}\right] .
$$

Da definição de soluções periódicas lentamente espiralantes e de (H.1), segue que $e^{\alpha t} x_{2}(t)$ e, portanto, $x_{2}(t)$, é estritamente decrescente para todo $t, t \in\left(q_{1}, q_{2}\right)$, o que contradiz o fato de $t_{2}$ ser ponto extremo de $x_{2}(t)$.

Analogamente provamos o resultado para $x_{1}(t), t \in\left[q_{2}, q_{4}\right]$.

Devido a simetria de $x(t)=\left(x_{1}(t), x_{2}(t)\right)$, o resultado é verdadeiro para todo $t \in\left[0, q_{4}+1\right]$, sendo $P=q_{4}+1$ o período mínimo de $x(t)=\left(x_{1}(t), x_{2}(t)\right)$.

Realizados estudos numéricos, notamos que $S^{*}$-soluções $x(t)=\left(x_{1}(t), x_{2}(t)\right)$ de $(2.1)_{\varepsilon}$, apresentam um bom comportamento ao longo de seu período. Não obstante o número de extremos relativos que suas coordenadas apresentam, existem números $c, d>0$, de modo que $x_{1}(t)$ e $x_{2}(t)$ oscilam em volta do zero de forma monótona, para todos os valores de $t$, tais que

$$
-c<x_{1}(t)<c \quad \text { e } \quad-d<x_{2}(t)<d .
$$

A seguinte definição é semelhante à propriedade $M$, dada por Mallet-Paret e Nussbaum em [1]. Inspira-se no comportamento descrito acima.

Para melhor compreensão dessa definição é conveniente referir-se à Figura 2.1. 


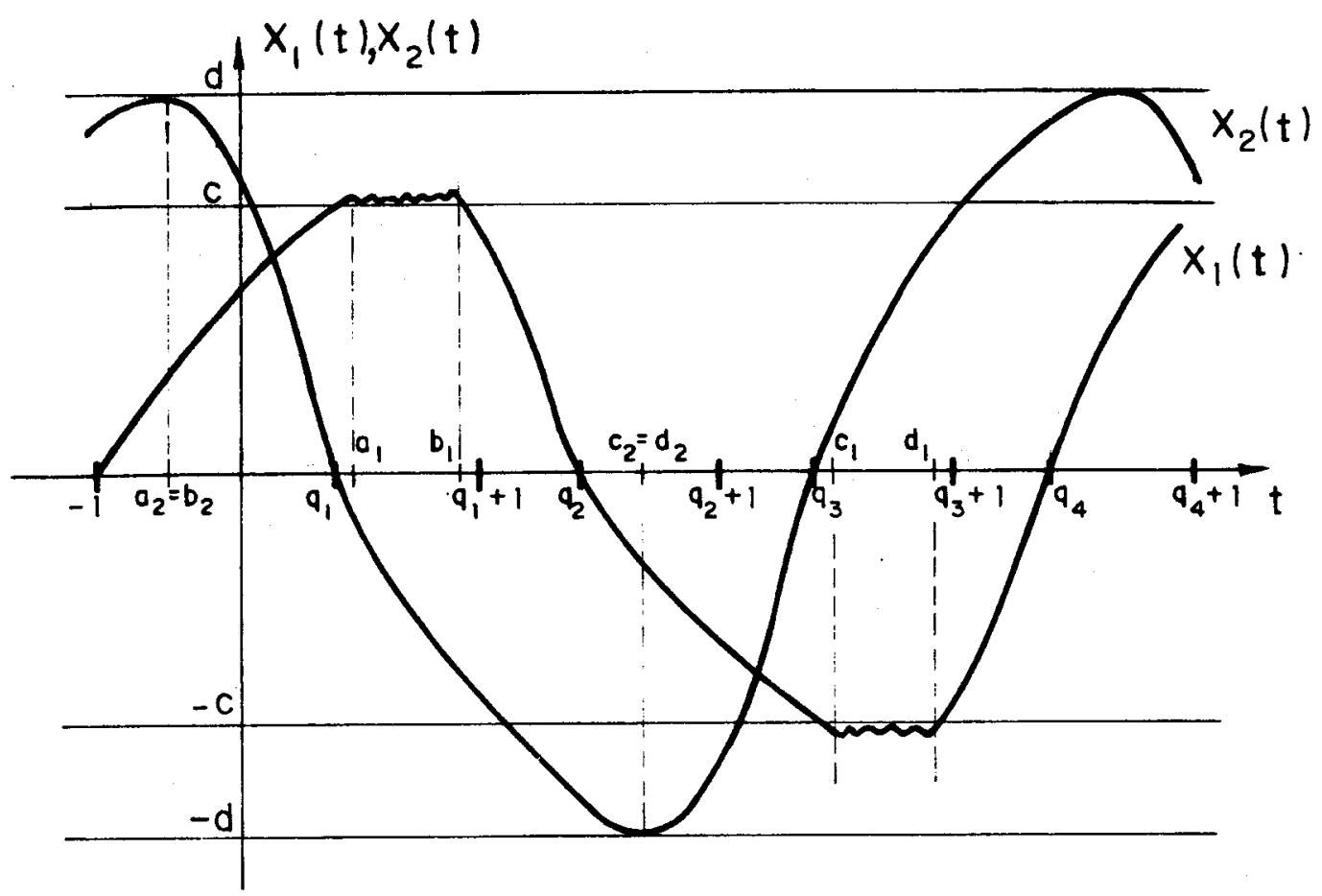

Figura 2.1: Uma $S^{*}$-solução satisfazendo a propriedade $J$.

Definição 2.1 Seja $x(t)=\left(x_{1}(t), x_{2}(t)\right)$ uma $S^{*}$-solução do sistema $(2.1)_{\varepsilon}$ com $-1, q_{2}$ e $q_{4}$ os primeiros zeros de $x_{1}(t)$ e $q_{1}$ e $q_{3}$ os primeiros zeros de $x_{2}(t)$.

Fixemos números $c, d>0$.

Dizemos que $x(t)$ satisfaz a propriedade $J$ para c e d se:

(1.1) $x_{1}(t)$ é monótona crescente em $\left[-1, a_{1}\right]$

(1.2) $x_{1}(t) \geq c$, se $a_{1}<t<b_{1}$

(1.3) $x_{1}(t)$ é monótona decrescente em $\left[b_{1}, c_{1}\right]$

(1.4) $x_{1}(t) \leq-c$, se $c_{1}<t<d_{1}$

(1.5) $x_{1}(t)$ é monótona crescente em $\left[d_{1}, q_{4}\right]$ 
(2.1) $x_{2}(t)$ é monótona crescente em $\left[-1, a_{2}\right]$

(2.2) $x_{2}(t) \geq d$, se $a_{2}<t<b_{2}$

(2.3) $x_{2}(t)$ é monótona decrescente em $\left[b_{2}, c_{2}\right]$

(2.4) $x_{2}(t) \leq-d$, se $c_{2}<t<d_{2}$

(2.5) $x_{2}(t)$ é monótona crescente em $\left[d_{2}, g_{4}\right]$

onde os números $a_{1}, b_{1}, c_{1}, d_{1}, a_{2}, b_{2}, c_{2}$ e $d_{2}$ são definidos como segue.

Sejam $t_{1}, t_{2} \in\left[-1, q_{4}\right]$, tais que

$$
\begin{aligned}
& x_{1}\left(t_{1}\right)=\max \left\{x_{1}(t) ; q_{1}<t<q_{1}+1\right\}, \quad e \\
& x_{2}\left(t_{2}\right)=\min \left\{x_{2}(t) ; q_{2}<t<q_{2}+1\right\} .
\end{aligned}
$$

- Se $x_{1}\left(t_{1}\right)>c \quad e \quad x_{2}\left(t_{2}\right)<-d$, defina

$$
\begin{aligned}
& \left\{\begin{array}{l}
a_{1}=\inf \left\{t \in\left[q_{1}, q_{1}+1\right] ; x_{1}(t)=c\right\}, \\
b_{1}=\sup \left\{t \in\left[q_{1}, q_{1}+1\right] ; x_{1}(t)=c\right\}, \quad e
\end{array}\right. \\
& \left\{\begin{array}{l}
c_{2}=\inf \left\{t \in\left[q_{2}, q_{2}+1\right] ; x_{2}(t)=-d\right\}, \\
d_{2}=\sup \left\{t \in\left[q_{2}, q_{2}+1\right] ; x_{2}(t)=-d\right\} .
\end{array}\right.
\end{aligned}
$$

- Se $x_{1}\left(t_{1}\right)>c$ e $x_{2}\left(t_{2}\right) \geq-d$, defina

$$
\left\{\begin{array}{l}
a_{1}, b_{1} \text { como em (2.2), e } \\
c_{2}=d_{2}=t_{2} .
\end{array}\right.
$$

- Se $x_{1}\left(t_{1}\right) \leq c$ e $x_{2}\left(t_{2}\right)<-d$, defina

$$
\left\{\begin{array}{l}
a_{1}=b_{1}=t_{1}, \quad e \\
c_{2}, d_{2} \text { como em (2.3). }
\end{array}\right.
$$

- Se $x_{1}\left(t_{1}\right) \leq c \quad$ e $x_{2}\left(t_{2}\right) \geq-d$, defina

$$
\left\{\begin{array}{l}
a_{1}=b_{1}=t_{1}, \quad e \\
c_{2}=d_{2}=t_{2} .
\end{array}\right.
$$


Similarmente, devido a simetria das $S^{*}$-soluções de $(2.1)_{\varepsilon}$ definimos $c_{1}, d_{1}, a_{2}$ $e b_{2}$.

Observação 2.1 Embora $t_{1}$ e $t_{2}$ possam não ser determinados de modo único, a propriedade $J$ está bem definida. Ainda, se $a_{1}=b_{1}$ e $c_{2}=d_{2}$, condições (1.2) e. (2.4), respectivamente, da propriedade $J$ estâo satisfeitas por vacuidade.

Para provarmos o próximo teorema faremos uso do seguinte lema dado em [1].

Lema 2.2 Seja $x:[\alpha, \beta] \rightarrow \mathbb{R}$ uma função $C^{1}$ tal que $x(\alpha)=x(\beta)=0$ e $x(t)>0$ quando $\alpha<t<\beta$. Suponhamos que c seja um número positivo e que existam números $t_{1}<t_{2}<t_{3}$ em $(\alpha, \beta)$ tais que

$$
\left\{\begin{array}{l}
x\left(t_{2}\right)<x\left(t_{j}\right) \text { para } j=1,3, \quad e \\
x\left(t_{2}\right)<c
\end{array}\right.
$$

Então existem números $t_{1}^{\prime}<t_{2}^{\prime}<t_{3}^{\prime}$ em $(\alpha, \beta)$ tais que

$$
\left\{\begin{array}{l}
x\left(t_{2}^{\prime}\right)<x\left(t_{j}^{\prime}\right) \leq c \text { e } \dot{x}\left(t_{j}^{\prime}\right) \geq 0 \text { para } j=1,3, \quad e \\
x\left(t_{2}^{\prime}\right) \leq x\left(t_{2}\right) \text { e } \dot{x}\left(t_{2}^{\prime}\right)=0
\end{array}\right.
$$

Prova: Ver Lema 3.1 de [1].

A Figura 2.2 ilustra o Lema 2.2 .

Observação 2.2 Sem perda de generalidade podemos definir a propriedade $J$ usando apenas uma constante positiva $c$. Isto é, podemos tomar $c=d$ na Definição 2.1. Desse modo, nos próximos resultados faremos uso dessa observação. 


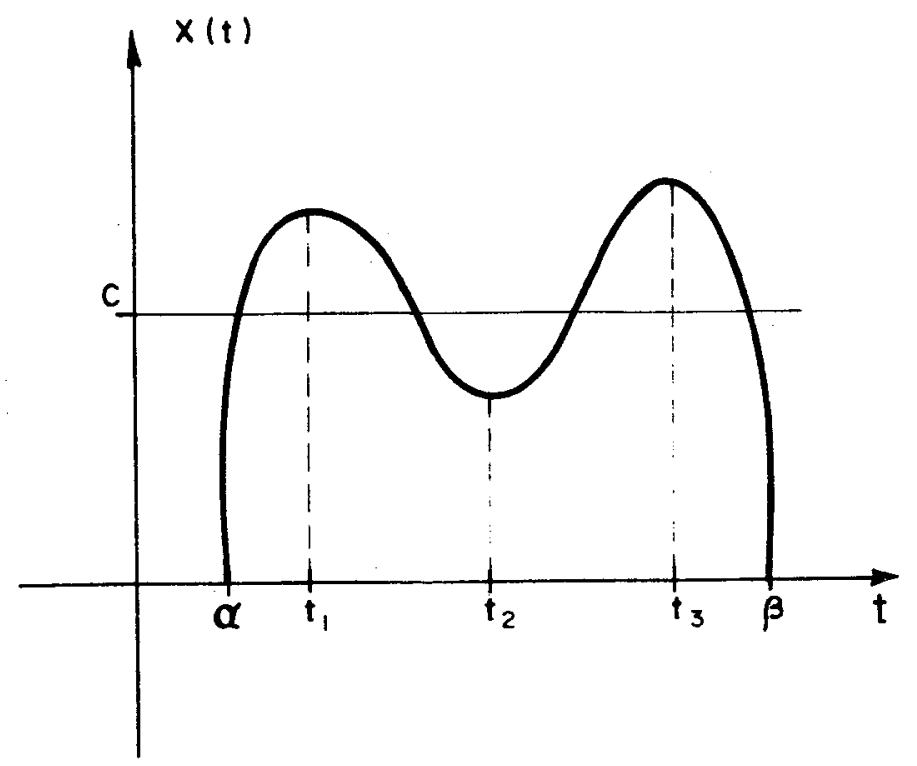

Figura 2.2

Ainda, precisamos da seguinte hipótese:
(H.5) $\lim _{x \rightarrow \pm \infty} \inf \left|f_{j}(x)\right|>0, j=1,2$, e
$f_{1}=-f_{2}$ numa vizinhança da origem.

Observação 2.3 Seja $F=\left(f_{1}, f_{2}\right)$ satisfazendo (H.1) e (H.2) e suponhamos que exista $0<A \leq M$, tal que, $f=f_{1}=-f_{2}$ em $I=[-A, A], f([-A, A]) \subseteq[-A, A]$, $f(-A)=-f(A)$ e $f(A)=A$. Nessas condições, o conjunto $Q=\left\{\left(x_{1}, x_{2}\right) \in\right.$ $\left.\mathbb{R}^{2} \mid-A \leq x_{1} \leq A \mathrm{e}-A \leq x_{2} \leq A\right\}$ é invariante para o sistema $(2.1)_{\varepsilon}$, e, para o estudo das soluções periódicas $x(t) \in Q$, a hipótese (H.5) é dispensável. De fato, 
definamos

$$
\bar{f}_{1}(x)= \begin{cases}f_{1}(-A), & \text { se } x \leq-A \\ f_{1}(x), & \text { se }-A \leq x \leq A \\ f_{1}(A), & \text { se } x \geq A\end{cases}
$$

e $\bar{f}_{1}=-\bar{f}_{2}$, para todo $x$. A função $\bar{F}=\left(\bar{f}_{1}, \bar{f}_{2}\right)$ satisfaz (H.1), (H.2) e (H.5) e segue-se que toda $S^{*}$-solução de $\varepsilon \dot{x}(t)=-x(t)+\bar{F}(x(t-1))$, satifaz $\left|x_{1}(t)\right|$, $\left|x_{2}(t)\right| \leq A$. Ela é portanto uma $S^{*}$-solução de $(2.1)_{\varepsilon}$, com $F=\left(f_{1}, f_{2}\right)$ satisfazendo as condições pedidas.

Teorema 2.1 Seja $x(t)=\left(x_{1}(t), x_{2}(t)\right)$ uma $S^{*}$-soluçấo do sistema $(2.1)_{\varepsilon}$, para um dado $\varepsilon>0, F=\left(f_{1}, f_{2}\right), f_{1}, f_{2}: \mathbb{R} \rightarrow \mathbb{R}$, diferenciáveis até ordem 2 na origem, funçôes impares, satisfazendo as hipóteses (H.1), (H.Q) e (H.5). Então $x(t)$ satisfaz a propriedade $J$ para alguma constante $c$, independente de $\varepsilon$.

Prova: Consideremos o sistema $(2.1)_{\varepsilon}$. As hipóteses (H.1) e (H.5) garantem a existência de uma constante positiva $c$, tal que

$$
\begin{aligned}
& \left\{\begin{array}{l}
f_{1}(x) \text { é monótona crescente em }[-c, c] \\
f_{1}(x) \geq c, \text { se } x \geq c \\
f_{1}(x) \leq-c, \text { se } x \leq-c
\end{array}\right. \\
& \left\{\begin{array}{l}
f_{2}(x) \text { é monótona decrescente em }[-c, c] \\
f_{2}(x) \geq c, \text { se } x \leq-c \\
f_{2}(x) \leq-c, \text { se } x \geq c .
\end{array}\right.
\end{aligned}
$$

Ainda, a hipótese (H.1) implica a existência de uma constante $\gamma>1$, tal que

$$
\left|f_{1}\left(f_{1}(x)\right)\right| \geq \gamma|x| \text { e }\left|f_{2}\left(f_{2}(x)\right)\right| \geq \gamma|x|
$$

$x \in[-c, c]$.

Assumamos, por contradição, que alguma $S^{*}$-solução $x(t)$ não satisfaça a propriedade $J$ entre $-c$ e $c$.

Vamos supor que as condições da propriedade $J$ para a coordenada $x_{1}(t)$ não estão satisfeitas, pois para $x_{2}(t)$ o procedimento é análogo e omitiremos a demonstração. 
Para simplificarmos, devido à simetria das $S^{*}$-soluções faremos a demonstração considerando $x_{1}(t)$ com $t$ variando num intervalo de comprimento igual a meio período, isto é, $t$ pertencente a $\left[-1, q_{2}\right]$.

O Lema 2.1 garante que $x_{1}(t)$ é monótona crescente em $\left[-1, q_{1}\right]$ e monótona decrescente em $\left[q_{1}+1, q_{2}\right]$.

Suponhamos por contradição que $x_{1}(t)$ não seja monótona crescente em $\left[q_{1}, a_{1}\right]$. Então existem números $t_{1}, t_{2}$ pertencentes a $\left(q_{1}, a_{1}\right)$, tais que

$$
\begin{aligned}
& q_{1}<t_{1}<t_{2}<a_{1}, \\
& c>x_{1}\left(t_{1}\right)>x_{1}\left(t_{2}\right),
\end{aligned}
$$

e definimos

$$
t_{3}=a_{1}
$$

Do mesmo modo, existem números $t_{j}, j=1,2,3$, satisfazendo as condições acima, se $x_{1}(t)$ não for monótona decrescente em $\left[b_{1}, q_{1}+1\right]$.

Agora, se existe $t_{0} \in\left(a_{1}, b_{1}\right) \subseteq\left[q_{1}, q_{1}+1\right]$, tal que, $x_{1}\left(t_{0}\right)<c$, definimos

$$
\begin{aligned}
& t_{1}=a_{1}, \\
& t_{3}=b_{1},
\end{aligned}
$$

e $t_{2}$, tal que, $x_{1}\left(t_{2}\right)=\min x_{1}(t), t \in\left(a_{1}, b_{1}\right) \subset\left[q_{1}, q_{1}+1\right]$.

Desse modo, acabamos de estabelecer a existência de três números $t_{1}<t_{2}<t_{3}$ pertencentes a $\left[-1, q_{2}\right]$ os quais satisfazem as hipóteses do Lema 2.2 para $x_{1}(t)$ $\operatorname{com}[\alpha, \beta]=\left[-1, q_{2}\right]$.

Definimos

$$
\begin{aligned}
S=\{ & t \in\left[q_{1}, q_{1}+1\right] \subset\left[-1, q_{2}\right] \mid 0<x_{1}(t)<c ; \\
& \exists t_{1}, t_{3} \in\left(q_{1}, q_{1}+1\right) \mid t_{1}<t<t_{3} \quad \mathrm{e} \\
& \left.x_{1}\left(t_{j}\right)>x_{1}(t), j=1,3\right\} .
\end{aligned}
$$

Seja

$$
\xi=\inf \left\{x_{1}(t) ; t \in S\right\}
$$


Notemos que $\xi>0$, pois $x_{1}(t) \neq 0$, para todo $t \in\left[q_{1}, q_{1}+1\right]$ e também que $\xi<c$.

Fixemos $t_{2} \in S$, tal que

$$
x_{1}\left(t_{2}\right)<\gamma \xi, \quad \gamma>1
$$

e seja $t_{1}<t_{2}<t_{3}$ como na definição de $S$.

O Lema 2.2 agora implica que existem pontos $t_{1}^{\prime}<t_{2}^{\prime}<t_{3}^{\prime}$ em $\left(-1, q_{2}\right)$ tais que

$$
\left\{\begin{array}{l}
x_{1}\left(t_{2}^{\prime}\right)<x_{1}\left(t_{j}^{\prime}\right) \leq c, \text { para } j=1,3, \\
0<x_{1}\left(t_{2}^{\prime}\right) \leq x_{1}\left(t_{2}\right), \\
\dot{x}_{1}\left(t_{2}^{\prime}\right)=0 \text { e } \dot{x}_{1}\left(t_{j}^{\prime}\right) \geq 0, \text { para } j=1,3 .
\end{array}\right.
$$

A primeira equação de $(2.1)_{\varepsilon}$ e condiçôes (2.9) e (2.11) implicam que

$$
0<x_{1}\left(t_{2}^{\prime}\right)=f_{1}\left(x_{2}\left(t_{2}^{\prime}-1\right)\right)<x_{1}\left(t_{j}^{\prime}\right) \leq f_{1}\left(x_{2}\left(t_{j}^{\prime}-1\right)\right), \text { para } j=1,3
$$

e portanto, $t_{j}^{\prime}-1 \in\left(-q_{3}+2 q_{1}, q_{1}\right)$ para $j=1,2,3$, pois $x_{2}\left(t_{j}^{\prime}-1\right)>0$ por (H.1). Ainda, (2.4) juntamente com (2.9) e (2.12) implicam que $0<x_{2}\left(t_{2}^{\prime}-1\right)<c$ e $x_{2}\left(t_{2}^{\prime}-1\right)<x_{2}\left(t_{j}^{\prime}-1\right)$, para $j=1,3$.

Chamamos $s_{j}=t_{j}^{\prime}-1+q_{3}-q_{1}$, para $j=1,2,3$. Então $s_{j} \in\left(q_{1}, q_{3}\right)$, $s_{1}<s_{2}<s_{3}$ e pela simetria das soluções $x_{2}\left(t_{j}^{\prime}-1\right)=-x_{2}\left(s_{j}\right), j=1,2,3$.

Logo, $x_{2}\left(s_{2}\right)>x_{2}\left(s_{j}\right), j=1,3$ e, pelo Lema $2.1, s_{j} \in\left(q_{2}, q_{2}+1\right) \subset\left[q_{1}, q_{3}\right]$, $j=1,2,3$.

Ainda, $0>x_{2}\left(s_{2}\right)>-c$.

Portanto os três números, $s_{1}, s_{2}, s_{3}$, satisfazem as hipóteses do Lema 2.2 para $-x_{2}(t), \operatorname{com}[\alpha, \beta]=\left[q_{1}, q_{3}\right]$. 
Dessa forma obtemos pontos $s_{1}^{\prime}<s_{2}^{\prime}<s_{3}^{\prime}$ pertencentes ao intervalo $\left(q_{1}, q_{3}\right)$ satisfazendo

$$
\left\{\begin{array}{l}
-c \leq x_{2}\left(s_{j}^{\prime}\right)<x_{2}\left(s_{2}^{\prime}\right), \text { para } j=1,3, \\
x_{2}\left(s_{2}\right) \leq x_{2}\left(s_{2}^{\prime}\right)<0, \\
\dot{x}_{2}\left(s_{2}^{\prime}\right)=0 \text { e } \dot{x}_{2}\left(s_{j}^{\prime}\right) \leq 0, \text { para } j=1,3 .
\end{array}\right.
$$

Chamamos $r_{j}=s_{j}^{\prime}-1$, para $j=1,2,3$. De modo análogo ao procedimento anterior e usando a segunda equação de $(2.1)_{\varepsilon}$ provamos que $r_{1}<r_{2}<r_{3}$ pertencem ao intervalo $\left[q_{1}, q_{1}+1\right] \subset\left[-1, q_{2}\right], x_{1}\left(r_{2}\right)<x_{1}\left(r_{j}\right)$, para $j=1,3$, e $0<x_{1}\left(r_{2}\right)<c$.

Dessa forma, estamos em condição de obter uma contradição, pois

$$
x_{1}\left(r_{2}\right) \geq \xi
$$

onde $\xi$ é dado em (2.7).

Usando o fato de $f_{1}, f_{2}$ serem funções impares, $f_{1}=-f_{2}$ em $I \supseteq[-c, c]$, suas propriedades estabelecidas em (2.4), (2.5) e (2.6), mais as propriedades dos pontos $t_{2}, t_{2}^{\prime}, s_{2}, s_{2}^{\prime}$ e $r_{2}$, obtidas acima, temos

$$
\begin{aligned}
x_{1}\left(t_{2}\right) & \geq x_{1}\left(t_{2}^{\prime}\right)=f_{1}\left(x_{2}\left(t_{2}^{\prime}-1\right)\right) \\
& =-f_{1}\left(x_{2}\left(s_{2}\right)\right)=f_{2}\left(x_{2}\left(s_{2}\right)\right) \\
& \geq f_{2}\left(x_{2}\left(s_{2}^{\prime}\right)\right)=f_{2}\left(f_{2}\left(x_{1}\left(s_{2}^{\prime}-1\right)\right)\right. \\
& =f_{2}\left(f_{2}\left(x_{1}\left(r_{2}\right)\right)\right. \\
& \geq \gamma x_{1}\left(r_{2}\right) .
\end{aligned}
$$

Portanto, de (2.16) e (2.17) obtemos $x_{1}\left(t_{2}\right) \geq \gamma \xi, \gamma>1$, o que contraria (2.8) e prova o teorema.

Nosso próximo resultado descreve um caso particular do Teorema 2.1, quando consideramos $f_{1}, f_{2}$ monótonas, em toda a imagem de uma $S^{*}$-solução.

Obtemos nesse caso um comportamento monotônico para estas soluções, descrito no corolário seguinte. 
Corolário 2.1 Seja $x(t)=\left(x_{1}(t), x_{2}(t)\right)$ uma $S^{*}$-solução do sistema $(2.1)_{\varepsilon}$, $F=\left(f_{1}, f_{2}\right), f_{1}, f_{2}: \mathbb{R} \rightarrow \mathbb{R}$, diferenciáveis até ordem 2 na origem, funções impares, satisfazendo as hipóteses (H.1) e (H.2). Suponhamos que exista $A>0$, $0<A \leq M$, tal que $f_{1}$ é monótona crescente e $f_{1}=-f_{2}$ em $[-A, A]$. Ainda, que $f_{1}, f_{2}$ satisfaçam

$$
\left|f_{1}\left(f_{1}(x)\right)\right|>|x| \text { e }\left|f_{2}\left(f_{2}(x)\right)\right|>|x|
$$

para todo $x \in(-A, A), x \neq 0$ e $f_{1}(x), f_{2}(x) \in[-A, A]$, se $x \in[-A, A]$. Então se $x(t)=\left(x_{1}(t), x_{2}(t)\right)$ é uma $S^{*}$-solução do sistema $(2.1)_{\varepsilon}$ para algum $\varepsilon>0$, $e$ se $\left|x_{1}(t)\right|,\left|x_{2}(t)\right| \leq A$, para todo $t, x(t)=\left(x_{1}(t), x_{2}(t)\right)$ satisfaz a propriedade $J$ para qualquer constante positiva $c \leq A$.

Prova: Precisamos fazer uso das seguintes desigualdades

$$
f_{1}\left(-m_{2}\right) \geq M_{1} \text { e } f_{2}\left(M_{1}\right) \leq m_{2},
$$

para $x_{1}, x_{2} \in[-A, A]$, onde $M_{1}=\max _{t} x_{1}(t)$ e $m_{2}=\min _{t} x_{2}(t)$.

Provemos a primeira delas. Suponhamos que $x_{1}(\xi)=M_{1}, \xi \in[-A, A]$. Logo $\dot{x}_{1}(\xi)=0$ e, pela primeira equação do sistema $(2.1)_{\varepsilon}$ e monotonicidade de $f_{1}$ nesse intervalo, temos

$$
M_{1}=x_{1}(\xi)=f_{1}\left(x_{2}(\xi-1)\right) \leq f_{1}\left(M_{2}\right)
$$

onde, $M_{2}=\max _{t} x_{2}(t), x_{2}(t) \in[-A, A]$. Ainda, com a hipótese da simetria de $f_{1}$ em $[-A . A]$ temos que $M_{2}=-m_{2}$, o que prova o resultado. A segunda desigualdade de (2.19) é provada similarmente.

Sejam $c_{1 n}, c_{2 n}$ sequências crescentes de números positivos satisfazendo

$$
\begin{gathered}
c_{1 n} \rightarrow M_{1}, c_{2 n} \rightarrow-m_{2}, \\
f_{2}\left(c_{1 n}\right) \leq-c_{2 n} \text { e } f_{1}\left(c_{2 n}\right) \geq c_{1 n} .
\end{gathered}
$$

Para assegurarmos (2.20) e (2.21), usamos (2.19) e tomamos $\left\{c_{1 n}\right\}$ estritamente crescente, $c_{1 n} \rightarrow M_{1}$ e $c_{2 n}<\min \left\{-f_{2}\left(c_{1 n}\right),-m_{2}\right\}$. Essa última condição é possível, uma vez que $-f_{2}\left(c_{1 n}\right) \rightarrow-f_{2}\left(M_{1}\right) \geq-m_{2}$.

Como $f_{1}=-f_{2}$ em $[-A, A]$, podemos tomar $c_{1 n}=c_{2 n}=c_{n}$. 
Observamos que as condições (2.4) e (2.5) do Teorema 2.1 estão satisfeitas para $c=c_{n}, n=1,2, \ldots$.

A condição (2.6) do Teorema 2.1 está garantida para $x_{1}, x_{2} \in\left[-c_{n}, c_{n}\right]$, $n=1,2, \ldots$, pela hipótese $(2.18)$.

Agora, olhando a Observação 2.3 estamos em condições de aplicar o Teorema 2.1 para $x_{1}, x_{2} \in\left[-c_{n}, c_{n}\right], n=1,2, \ldots$, onde $c_{n}$ converge para os extremos das funções $x_{1}, x_{2} \in[-A, A]$. Dessa forma, Teorema 2.1 implica o resultado.

Nosso objetivo agora é obter estimativas para o período das $S^{*}$-soluções. O Lema 2.3 dá uma primeira limitação para o período mínimo das $S^{*}$-soluções fornecidas pelo Teorema 1.2.

Lema 2.3 Seja $x(t)=\left(x_{1}(t), x_{2}(t)\right) \quad$ uma $S^{*}$-solução do sistema $(2)_{\alpha}$, $F=\left(f_{1}, f_{2}\right), f_{1}, f_{2}: \mathbb{R} \rightarrow \mathbb{R}$, diferenciáveis até ordem 2 na origem, funções ímpares, satisfazendo as hipóteses (H.1)-(H.4). Então, existe um número $\bar{P}>0$, dependendo somente da $F=\left(f_{1}, f_{2}\right)$, tal que, se $x(t)$ é uma $S^{*}$-solução de $(2)_{\alpha}$, então seu periodo mínimo, $P=q_{4}+1$, satisfaz $P<\bar{P}$.

Prova: As hipóteses (H.1) e (H.2) implicam na existência de uma constante positiva $\Omega>1$ que depende somente de $F=\left(f_{1}, f_{2}\right)$, tal que

$$
\begin{cases}\left|f_{1}(x)\right| \leq \Omega|x|, & x \in \mathbb{R}, \\ \left|f_{2}(x)\right| \leq \Omega|x|, & x \in \mathbb{R} .\end{cases}
$$

Sejam $\bar{\alpha}$ dado no Teorema $1.2, \alpha>\bar{\alpha}$ e $x(t)$ uma $S^{*}$-solução do sistema $(2)_{\alpha}$.

Da primeira equação de $(2)_{\alpha}$, temos que

$$
\left(e^{\alpha\left(t-\left(q_{1}+1\right)\right)} x_{1}(t)\right)^{\prime} \leq 0, \text { se } q_{1}+1 \leq t \leq q_{2}
$$

e portanto,

$$
0<x_{1}(t) \leq e^{-\alpha\left(t-\left(q_{1}+1\right)\right)} x_{1}\left(q_{1}+1\right)<e^{-\bar{\alpha}\left(t-\left(q_{1}+1\right)\right)} M_{1},
$$

se $q_{1}+1 \leq t \leq q_{2}$ 
onde

$$
M_{1}=\max _{q_{1}<t<q_{1}+1} x_{1}(t)
$$

A relação $(2.23)$, implica

$$
0<x_{1}(t)<e^{-\bar{\alpha}\left(q_{2}-\left(q_{1}+2\right)\right)} M_{1} \text {, se } q_{2}-1 \leq t \leq q_{2} .
$$

Observando que se $t \in\left[q_{2}-1, q_{1}+1\right]$ esta desigualdade segue de $0<x_{1}(t) \leq M_{1}$ e $-\bar{\alpha}\left(q_{2}-\left(q_{1}+2\right)\right)>0$.

Usando essa desigualdade e (2.22), e integrando a segunda equaçâo de $(2)_{\alpha}$ de $q_{2}$ a $t, t \in\left[q_{2}, q_{2}+1\right]$, temos

$$
\begin{aligned}
e^{\alpha\left(t-q_{2}\right)}\left|x_{2}(t)\right| & \leq \int_{q_{2}}^{t} e^{\alpha\left(s-q_{2}\right)} \mid f_{2}\left(x_{1}(s-1) \mid d s\right. \\
& \leq\left[e^{\alpha\left(t-q_{2}\right)}-1\right] \Omega e^{-\bar{\alpha}\left[q_{2}-\left(g_{1}+2\right)\right]} M_{1} .
\end{aligned}
$$

Essa relação implica

$$
\left|m_{2}\right|<e^{-\bar{\alpha}\left[g_{2}-\left(g_{1}+2\right)\right]} \Omega M_{1},
$$

onde

$$
m_{2}=\min _{q_{2}<t<q_{2}+1} x_{2}(t)
$$

Pela simetria, temos

$$
M_{2}<e^{-\bar{\alpha}\left[q_{2}-\left(q_{1}+2\right)\right]} \Omega M_{1} .
$$

Um argumento semelhante mostra que

$$
M_{1}<e^{-\bar{\alpha}\left(q_{1}-1\right)} \Omega M_{2} .
$$

De $(2.24)$ e $(2.25)$, temos

$$
M_{1}<e^{-\bar{\alpha}\left(\left(q_{2}+1\right)-4\right)} \Omega^{2} M_{1} .
$$

Como $M_{1}>0$, temos que $e^{-\bar{\alpha}\left(\left(q_{2}+1\right)-4\right)} \Omega^{2}>1$, 
portanto

$$
\begin{gathered}
\left(q_{2}+1\right)-4<\frac{2 \ln \Omega}{\bar{\alpha}} \\
q_{2}+1<\frac{2 \ln \Omega}{\bar{\alpha}}+4
\end{gathered}
$$

Chamando

$$
\bar{p}=\frac{2 \ln \Omega}{\bar{\alpha}}+4
$$

temos que $p=q_{2}+1$ é tal que

$$
p<\bar{p}
$$

E, portanto,

$$
P<\bar{P}
$$

onde

$$
\bar{P}=\frac{4 \ln \Omega}{\bar{\alpha}}+8
$$

O próximo teorema fornece estimativas mais finas para o período. É oportuno observarmos que a hipótese (H.5) contém (H.4).

Teorema 2.2 Seja $x(t)=\left(x_{1}(t), x_{2}(t)\right)$ uma $S^{*}$-solução do sistema $(2.1) \varepsilon$, $F=\left(f_{1}, f_{2}\right), f_{1} f_{2}: \mathbb{R} \rightarrow \mathbb{R}$, diferenciáveis até ordem 2 na origem, funçôes impares, satisfazendo as hipóteses (H.1) - (H.3) e (H.5). Existe constante positiva $\bar{C}$, dependendo somente de $F=\left(f_{1}, f_{2}\right)$, tal que, se $x(t)$ é uma $S^{*}$-solução de $(2.1)_{\varepsilon}$ para algum $\varepsilon>0$, então seu período minimo $P=P_{\varepsilon}$ satisfaz a desigualdade

$$
4<P_{\varepsilon}<4(1+\bar{C} \varepsilon)
$$

Prova: Da definição de $S^{*}$-solução, seguem as cotas inferiores $P_{\varepsilon}=q_{4}+1>4$ e $p_{\varepsilon}=q_{2}+1>2$.

Portanto, todo nosso esforço será concentrado para obter a cota superior $p_{\varepsilon}<2(1+\bar{C} \varepsilon)$ e consequentemente a cota superior $P_{\varepsilon}<4(1+\bar{C} \varepsilon)$.

As hipóteses (H.1) , (H.2) e (H.5) implicam na existência de constantes positivas $c, \gamma>1$ e $\Omega>1$ que dependem somente de $F=\left(f_{1}, f_{2}\right)$, tais que valem 
(2.4), (2.5), (2.6) e (2.22) e, além disso,

$$
\left\{\begin{array}{l}
\left|f_{1}(x)\right| \geq \gamma c, \quad|x| \geq c \\
\left|f_{2}(x)\right| \geq \gamma c, \quad|x| \geq c
\end{array}\right.
$$

Sejam $x(t)$ uma $S^{*}$-solução de $(2.1)_{\varepsilon}$ para algum $\varepsilon>0, M_{1}=\max _{t} x_{1}(t)$ e $\left|m_{2}\right|=\left|\min _{t} x_{2}(t)\right|$.

Definimos

$$
\Lambda=\max \left\{\frac{M_{1}}{\Omega}, \frac{\left|m_{2}\right|}{\Omega}\right\}
$$

e

$$
\bar{c}=\min \left\{c, \frac{\Lambda}{2 \Omega}\right\} \text {. }
$$

Consequentemente $x(t)$ satisfaz a Propriedade $J$ entre $-\bar{c}$ e $\bar{c}$ e $\left|f_{1}(x)\right|$, $\left|f_{2}(x)\right| \geq \gamma \bar{c}$, se $|x| \geq \bar{c}$.

Afirmamos que se $x(t)=\left(x_{1}(t), x_{2}(t)\right)$ é uma $S^{*}$-solução do sistema $(2.1) \varepsilon$, para algum $\varepsilon>0$, então

$$
M_{1} \leq \Omega\left|m_{2}\right|
$$

De fato, suponhamos que $x_{1}(t)$ alcança seu máximo no ponto $t_{1 M}$. Então a primeira equação de $(2.1)_{\varepsilon}$ e a simetria das $S^{*}$-soluções implicam que

$$
M_{1}=x_{1}\left(t_{1 M}\right)=f_{1}\left(x_{2}\left(t_{1 M}-1\right)\right) \leq \Omega\left|x_{2}\left(t_{1 M}-1\right)\right| \leq \Omega\left|m_{2}\right| .
$$

Analogamente, temos

$$
\left|m_{2}\right| \leq \Omega M_{1}
$$

Portanto, se $\Lambda=\frac{M_{1}}{\Omega}$ ou $\Lambda=\frac{\left|m_{2}\right|}{\Omega}$, considerando-se (2.33) ou (2.34), respectivamente, temos

$$
\begin{gathered}
M_{1} \geq \Lambda>\bar{c}, \\
m_{2} \leq-\Lambda \leq-\bar{c} .
\end{gathered}
$$


Logo, pela simetria das soluções $x(t)$, essas contém o intervalo $[-\bar{c}, \bar{c}]$ para todo $t \in\left[-1, q_{4}\right]$.

Sejam $a_{1}$ e $b_{1}$ o primeiro e último tempo respectivemente em $\left[q_{1}, q_{1}+1\right]$, tais que $x_{1}\left(a_{1}\right)=x_{1}\left(b_{2}\right)=\bar{c}$; e similarmente sejam $c_{2}$ e $d_{2}$ o primeiro e último tempo em $\left[q_{2}, q_{2}+1\right]$, tal que $x_{2}\left(c_{2}\right)=x_{2}\left(d_{2}\right)=-\bar{c}$, como na definição da propriedade $J$, com $c=d=\bar{c}$. Observe-se que $x_{1}(t) \geq \bar{c}$, se $a_{1} \leq t \leq b_{1}$ e $x_{2}(t) \leq-\bar{c}$, se $c_{2} \leq t \leq d_{2}$.

Afirmamos que existe uma constante $C_{*}$, independente de $\varepsilon$ e da solução $x(t)$, tal que

$$
b_{1}-a_{1} \geq C_{*} \varepsilon \Longrightarrow\left[1+a_{1}+C_{*} \varepsilon, 1+b_{1}\right] \subseteq\left[c_{2}, d_{2}\right]
$$

e similarmente,

$$
d_{2}-c_{2} \geq C_{*} \varepsilon \Longrightarrow\left[1+c_{2}+C_{*} \varepsilon, 1+d_{2}\right] \subseteq\left[a_{1}+\left(q_{2}+1\right), b_{1}+\left(q_{2}+1\right)\right] .
$$

Provaremos (2.37). Analogamente prova-se (2.38).

Seja

$$
C_{*}=\max \left\{\ln \frac{\bar{M}_{1}+\gamma c}{(\gamma-1) c}, \ln \frac{\left|\bar{m}_{2}\right|+\gamma c}{(\gamma-1) c}, \ln \frac{2 \Omega^{2}+\gamma}{\gamma-1}\right\}
$$

onde $\bar{M}_{1}=\sup _{\varepsilon} M_{1}$ e $\left|\bar{m}_{2}\right|=\sup _{\varepsilon}\left|m_{2}\right|$.

Suponhamos que $b_{1}-a_{1} \geq C_{*} \varepsilon$.

Então para $t \in\left[1+a_{1}, 1+a_{1}+C_{*} \varepsilon\right]$ temos $x_{1}(t-1) \geq \bar{c}$ e portanto, da segunda equação de (2.1) e de (2.30), temos

$$
\varepsilon \dot{x}_{2}(t) \leq-x_{2}(t)-\gamma \bar{c} .
$$

Integrando (2.39) em $t, t \in\left[1+a_{1}, 1+a_{1}+C_{*} \varepsilon\right]$, temos

$$
x_{2}\left(1+a_{1}+C_{*} \varepsilon\right) \leq x_{2}\left(1+a_{1}\right) e^{-C_{*}}-\left(1-e^{-C_{*}}\right) \gamma \bar{c}
$$

e usando (2.31) e (2.32), obtemos

$$
x_{2}\left(1+a_{1}+c_{*} \varepsilon\right) \leq-\bar{c}
$$


Da propriedade $J$ e da definição de $c_{2}$, segue que

$$
1+a_{1}+c_{*} \varepsilon \geq c_{2}
$$

Vamos mostrar que

$$
1+b_{1} \leq d_{2}
$$

Se (2.43) não fosse verdadeira, então teríamos

$$
d_{2}<1+b_{1} \leq q_{1}+1+1<q_{2}+1,
$$

o que implica, pela definição de $d_{2}$, que

$$
\begin{gathered}
x_{2}\left(1+b_{1}\right) \geq-\bar{c}, \mathrm{e} \\
\dot{x}_{2}\left(1+b_{1}\right) \geq 0 .
\end{gathered}
$$

Como $x_{1}\left(b_{1}\right)=\bar{c}$, teríamos da segunda equação de $(2.1)_{\varepsilon}$, de $(2.44)$ e $(2.30)$ que

$$
\varepsilon \dot{x}_{2}\left(1+b_{1}\right)<0 .
$$

Contradizendo (2.45), provando-se assim (2.43).

De (2.42) e (2.43) temos a inclusâo (2.37).

Consideremos agora dois casos:

\section{Caso I:}

$$
b_{1}-a_{1} \geq 2 C_{*} \varepsilon \quad \text { ou } \quad d_{2}-c_{2} \geq 2 C_{*} \varepsilon .
$$

De acordo com o que acabamos de ver, valem (2.37) e (2.38).

De modo que

$$
p_{\varepsilon} \leq 2\left(1+C_{*} \varepsilon\right)
$$

o que prova o teorema nesse caso. 


\section{Caso II:}

$$
\begin{gathered}
b_{1}-a_{1}<2 C_{*} \varepsilon, \quad \mathrm{e} \\
d_{2}-c_{2}<2 C_{*} \varepsilon .
\end{gathered}
$$

Quando $2 C_{*} \varepsilon>1$ usamos o limite (2.26) do Lema 2.3, para obtermos

$$
p_{\varepsilon}<2\left(1+p^{\prime} C_{*} \varepsilon\right)
$$

onde $p^{\prime}=\bar{p}-2$.

Assumamos agora $\varepsilon>0$, suficientemente pequeno, tal que

$$
2 c_{*} \varepsilon \leq 1
$$

Vamos mostrar primeiro que:

$$
\begin{gathered}
c_{2}-b_{1} \leq 1, \quad \mathrm{e} \\
a_{1}+\left(q_{2}+1\right)-d_{2} \leq 1,
\end{gathered}
$$

assumindo as condições (2.47) e (2.48).

Provemos (2.51).

Assumamos, por contradição, que $1+b_{1}<c_{2}$ e seja $t_{2 m} \in\left[c_{2}, d_{2}\right]$, tal que $x_{2}\left(t_{2 m}\right)=m_{2}$.

A desigualdade (2.48) implica que o comprimento do intervalo $\left[c_{2}, d_{2}\right]$ é menor que 1 , portanto, $t_{2 m}-1<c_{2}$.

Também $t_{2 m}-1 \geq c_{2}-1>b_{1}$ e portanto, pela propriedade $J,\left|x_{1}(t-1)\right| \leq \bar{c}$ para $1+b_{1} \leq t \leq t_{2 m}$.

Portanto, da segunda equação de $(2.1)_{\varepsilon}$ e de (2.22), para $1+b_{1} \leq t \leq t_{2 m}$ vem

$$
\varepsilon \dot{x}_{2}(t) \geq-x_{2}(t)-\Omega \bar{c}
$$

Integrando (2.53) de $1+b_{1}$ a $t_{2 m}$, temos

$$
x_{2}\left(t_{2 m}\right) \geq e^{-k} x_{2}\left(1+b_{1}\right)-\left(1-e^{-k}\right) \Omega \bar{c}>-(1+\Omega) \bar{c}
$$


onde $k=\left(t_{2 m}-b_{1}-1\right) / \varepsilon>0$. Portanto,

$$
\left|x_{2}\left(t_{2 m}\right)\right|<(1+\Omega) \bar{c} .
$$

Por outro lado, (2.36) implica que $\left|x_{2}\left(t_{2 m}\right)\right| \geq \Lambda$ e portanto,

$$
\Lambda<(1+\Omega) \bar{c} .
$$

Agora (2.54) e a definição (2.32) são inconsistentes com o fato de que $\Omega>1$. Essa contradição prova (2.51).

Analogamente provamos (2.52).

Para completarmos a prova, basta adicionarmos as desigualdades (2.47), (2.48), (2.51) e (2.52), obtendo-se

$$
p_{\varepsilon}<2\left(1+2 C_{*} \varepsilon\right) .
$$

Finalizando, os limites (2.46), (2.49) e (2.55) para $p_{\varepsilon}$ implicam que $P_{\varepsilon}<4(1+\bar{C} \varepsilon), \operatorname{com} \bar{C} \geq \max \left\{2 C_{*}, p^{\prime} C_{*}\right\}$. 


\section{Capítulo 3}

\section{Equações de camada de transição}

Consideremos o sistema de equações diferenciais autônomas

$$
\left\{\begin{array}{l}
\varepsilon \dot{x}_{1}(t)=h_{1}\left(x_{1}(t), x_{2}(t-1)\right) \\
\varepsilon \dot{x}_{2}(t)=h_{2}\left(x_{2}(t), x_{1}(t-1)\right)
\end{array}\right.
$$

onde $h_{1}, h_{2}: \mathbb{R}^{2} \rightarrow \mathbb{R}$

Seja $x(t)=\left(x_{1}(t), x_{2}(t)\right)$ solução periódica de $(3.1)$, para $\varepsilon \neq 0$, de período mínimo $P$, e definamos $r \in \mathbb{R}$ por

$$
p=2(1+\varepsilon r)
$$

onde $p$ é metade do período mínimo $P$.

Definamos as funções $y_{j}(t), j=1,2,3,4$, por

$$
\begin{aligned}
& y_{1}(t)=x_{1}(-\varepsilon t), \\
& y_{2}(t)=x_{2}(p / 2-\varepsilon t), \\
& y_{3}(t)=x_{1}(p-\varepsilon t), \\
& y_{4}(t)=x_{2}(3 / 2 p-\varepsilon t) .
\end{aligned}
$$


É fácil verificar que as funções $y_{j}(t), j=1,2,3,4$, satisfazem o seguinte sistema de equações

$$
\left\{\begin{array}{l}
\dot{y}_{1}(t)=-h_{1}\left(y_{1}(t), y_{4}(t-r)\right) \\
\dot{y}_{2}(t)=-h_{2}\left(y_{2}(t), y_{1}(t-r)\right) \\
\dot{y}_{3}(t)=-h_{1}\left(y_{3}(t), y_{2}(t-r)\right) \\
\dot{y}_{4}(t)=-h_{2}\left(y_{4}(t), y_{3}(t-r)\right)
\end{array}\right.
$$

Notemos que o parâmetro $r$ definido em (3.2), está ligado ao período da solução, $x(t)=\left(x_{1}(t), x_{2}(t)\right)$, de $(3.1)$.

Aplicando esta mudança de variável ao nosso sistema $(2.1)_{\varepsilon}$, considerando que $f_{1}, f_{2}$ são ímpares e a simetria das $S^{*}$-soluçôes de $(2.1)_{\varepsilon}$, temos (3.3) reduzido a apenas duas equações. De fato, devido à simetria das $S^{*}$-soluções, temos,

$$
\begin{aligned}
& y_{3}(t)=x_{1}(p-\varepsilon t)=-x_{1}(-\varepsilon t)=-y_{1}(t), \\
& y_{4}(t)=x_{2}(3 / 2 p-\varepsilon t)=-x_{2}(p / 2-\varepsilon t)=-y_{2}(t) .
\end{aligned}
$$

Substituindo essas relações em (3.3), vem

$$
\begin{aligned}
& \dot{y}_{1}(t)=-h_{1}\left(y_{1}(t),-y_{2}(t-r)\right), \\
& \dot{y}_{2}(t)=-h_{2}\left(y_{2}(t), y_{1}(t-r)\right), \\
& \dot{y}_{1}(t)=h_{1}\left(-y_{1}(t), y_{2}(t-r)\right), \\
& \dot{y}_{2}(t)=h_{2}\left(-y_{2}(t),-y_{1}(t-r)\right) .
\end{aligned}
$$

Este raciocínio aplicado ao sistema $(2.1)_{\varepsilon}$ leva a

$$
\begin{aligned}
& \dot{y}_{1}(t)=y_{1}(t)-f_{1}\left(-y_{2}(t-r)\right), \\
& \dot{y}_{2}(t)=y_{2}(t)-f_{2}\left(y_{1}(t-r)\right), \\
& \dot{y}_{1}(t)=y_{1}(t)+f_{1}\left(y_{2}(t-r)\right), \\
& \dot{y}_{2}(t)=y_{2}(t)+f_{2}\left(-y_{1}(t-r)\right) .
\end{aligned}
$$

Devido às simetrias das funções $f_{1}$ e $f_{2}$, as equações acima se reduzem a

$$
\left\{\begin{array}{l}
\dot{y}_{1}(t)=y_{1}(t)+f_{1}\left(y_{2}(t-r)\right) \\
\dot{y}_{2}(t)=y_{2}(t)-f_{2}\left(y_{1}(t-r)\right)
\end{array}\right.
$$


Definição 3.1 O sistema (3.4) é denominado sistema de equaçôes de camada de transiçâo associado ao sistema $(2.1)_{\varepsilon}$.

$\mathrm{O}$ resultado seguinte, que é análogo à Proposição 3.1 de [1], deixa clara a relação entre as equaçôes $(2.1)_{\varepsilon}$ e as equaçôes (3.4).

Proposição 3.1 Suponhamos que $F=\left(f_{1}, f_{2}\right)$, satisfaça as hipóteses (H.1) (H.3) e (H.5) com $f_{1}, f_{2}: \mathbb{R} \rightarrow \mathbb{R}$, diferenciáveis até ordem 2 na origem $e$ impares.

a) Sejam $\varepsilon>0, x(t)=\left(x_{1}(t), x_{2}(t)\right)$ uma $S^{*}$-solução das equaçôes $(2.1) \varepsilon$, de periodo mínimo $P=q_{4}+1, q_{4}$ um zero de $x_{1}, \theta \in \mathbb{R}$.

Definamos

$$
\begin{gathered}
y_{1}(t)=x_{1}(\theta-\varepsilon t), \\
y_{2}(t)=x_{2}(\theta+p / 2-\varepsilon t),
\end{gathered}
$$

onde $p$ é metade do período $P$.

Então $y(t)=\left(y_{1}(t), y_{2}(t)\right)$ satisfaz as equações de camada de transição (3.4) onde $r>0$ é dado por

$$
p=2(1+\varepsilon r)
$$

b) Sejam $\varepsilon_{n}>0, x_{n}(t)=\left(x_{1 n}(t), x_{2 n}(t)\right), p_{n}$ e $\theta_{n} \in \mathbb{R}, n=1,2, \ldots$, sequências tais que $\varepsilon_{n} \rightarrow 0$, e $x_{n}(t)$ é uma $S^{*}$-solução do sistema $(2.1) \varepsilon_{n}$ de periodo mínimo $P_{n}$ e $p_{n}$ metade do periodo minimo. Sejam $r_{n}>0, y_{n}(t)=$ $\left(y_{1 n}(t), y_{2 n}(t)\right)$ definidos por:

$$
\begin{gathered}
p_{n}=2\left(1+\varepsilon_{n} r_{n}\right), \\
y_{1 n}(t)=x_{1 n}\left(\theta_{n}-\varepsilon_{n} t\right), \quad e \\
y_{2 n}(t)=x_{2 n}\left(\theta_{n}+p_{n / 2}-\varepsilon_{n} t\right) .
\end{gathered}
$$


Entâo $r_{n}$ é uma sequência limitada, e as sequências de funções $y_{1_{n}}(t) \epsilon$ $y_{2 n}(t)$ são equicontínuas $e$ uniformemente limitadas.

Tomando os limites

$$
\begin{aligned}
& r_{n} \rightarrow r \geq 0 \\
& y_{1 n}(t) \rightarrow y_{1}(t) \quad \text { e } \quad y_{2 n}(t) \rightarrow y_{2}(t)
\end{aligned}
$$

unifomes em intervalos compactos, para alguma subsequência $n=n_{j} \rightarrow \infty$, as funções $y_{1}(t)$ e $y_{2}(t)$ são $C^{1}$ e satisfazem as equações de camada de transição (3.4), para todo $t \in \mathbb{R}$. Ainda, para todo $t \in \mathbb{R}$, temos

$$
\left|y_{1}(t)\right| \leq M \quad e \quad\left|y_{2}(t)\right| \leq M
$$

onde $M>0$ é dado em (H.2).

Também, a subsequência pode ser tomada de modo que $\dot{y}_{1 n}(t)$ e $\dot{y}_{2 n}(t)$ convirjam, respectivamente, para $\dot{y}_{1}(t)$ e $\dot{y}_{2}(t)$, uniformemente em intervalos compactos.

c) Se $y_{1}(t)$ é obtida como em b) então $y_{1}(t)$ tem no máximo uma troca de sinal sobre o eixo real. Isto é, não existem $t_{1}<t_{2}<t_{3}$ tais que $y_{1}\left(t_{1}\right) y_{1}\left(t_{2}\right)<0$ e $y_{1}\left(t_{2}\right) y_{1}\left(t_{3}\right)<0$. O mesmo é verdadeiro para $y_{2}(t)$, se obtida como em $\left.b\right)$.

d) Se $y_{1}(t)$ e $y_{2}(t)$ são obtidas como em b) elas têm sinais opostos quando $t \rightarrow \infty$. Do mesmo modo, $y_{1}(t)$ e $y_{2}(t)$ têm sinais opostos quando $t \rightarrow-\infty$.

e) Se $y_{1}(t)$ e $y_{2}(t)$ sâo obtidias como em b) e se $\lim _{t \rightarrow \infty} y_{1}(t)=L$ existe para algum número real $L$, então $\lim _{t \rightarrow \infty} y_{2}(t)=f_{2}(L)$ e $f_{1}\left(f_{2}(L)\right)=-L$. O correspondente resultado é válido quando $t \rightarrow-\infty$. Podemos também permutar $y_{1}(t)$ com $y_{2}(t)$ e $f_{1}$ com $f_{2}$, respectivamente.

Prova: Embora e) no nosso caso se apresente com uma leve modificação, a prova segue análoga à Proposição 3.1 de [1].

Observação $3.1 \mathrm{Em} \mathrm{d}$ ), notemos que os sinais de $y_{1}(t)$, quando $t \rightarrow+\infty \mathrm{e}$ $t \rightarrow-\infty$, podem ou não ser diferentes, dependendo se $y_{1}(t)$ passou ou não por uma mudança de sinal. $O$ mesmo é verdadeiro para $y_{2}(t)$. 
Definição 3.2 Sejam $\varepsilon_{n} \rightarrow 0_{+}, x_{n}(t)=\left(x_{1 n}(t), x_{2 n}(t)\right)$, uma $S^{*}$-solução do sistema $(2.1)_{\varepsilon_{n}}, \theta_{n} \in \mathbb{R}$ e $F=\left(f_{1}, f_{2}\right)$, satisfazendo (H.1) - (H.3) e (H.5), com $f_{1}, f_{2}: \mathbb{R} \rightarrow \mathbb{R}$, diferenciáveis até ordem 2 na origem e impares. Sejam ainda, $y(t)=\left(y_{1}(t), y_{2}(t)\right)$ e $r$, obtidos pelo processo limite da segunda parte da Proposição 3.1. Então $(y(t), r)$ é chamada uma soluçẫo das equações de camada de transição associada a sequência $\left(\varepsilon_{n}, x_{n}(t), \theta_{n}\right)$.

Observação 3.2 Notemos que essa definição admite a possibilidade de $(y(t), r)$ não ser única.

As considerações apresentadas a seguir estão na linha dos comentários de Mallet-Paret e Nussbaum [1], referentes ao enfoque dado no estudo das equações da camada de transição.

Seja o sistema linear

$$
\left\{\begin{array}{l}
\dot{y}_{1}(t)=y_{1}(t)+\delta y_{2}(t-r) \\
\dot{y}_{2}(t)=y_{2}(t)+\delta y_{1}(t-r)
\end{array}\right.
$$

obtido das equações de camada de transição (3.4), linearizados na origem, lembrando que nossas hipóteses sobre $f_{1}$ e $f_{2}$ implicam $\delta=\delta_{1}=-\delta_{2}>0$.

No próximo lema as equações de camada de transição são vistas como uma perturbação não-autônoma do sistema linear (3.10), isto é, como

$$
\dot{W}(t)=L\left(W_{t}\right)+\rho(t)
$$

onde

$$
W(t)=\left(\begin{array}{l}
y_{1}(t) \\
y_{2}(t)
\end{array}\right), W_{t}(s)=W(t+s) \quad \text { para }-r \leq s \leq 0,
$$

$L: C\left([-r, 0], \mathbb{R}^{2}\right) \rightarrow \mathbb{R}^{2}$ é o operador linear

$$
L\left(\begin{array}{l}
\varphi \\
\psi
\end{array}\right)=\left(\begin{array}{l}
\varphi(0)+\delta \psi(-r) \\
\psi(0)+\delta \varphi(-r)
\end{array}\right) \text { para } \varphi, \psi \in C[-r, 0]
$$


onde $\rho(t)$ é dado por

$$
\rho(t)=\left(\begin{array}{r}
R_{1}\left(y_{2}(t-r)\right) \\
-R_{2}\left(y_{1}(t-r)\right)
\end{array}\right)
$$

e,

$$
\left\{\begin{array}{l}
f_{1}\left(y_{2}\right)=\delta y_{2}+R_{1}\left(y_{2}\right) \\
f_{2}\left(y_{1}\right)=-\delta y_{1}+R_{2}\left(y_{1}\right)
\end{array}\right.
$$

Seguindo Hale (4), podemos associar a qualquer autosolução $W(t)=\omega e^{\xi t}$, da equação diferencial com retardamento linear autônoma em $\mathbb{R}^{n}$, uma projeção canônica sobre o autoespaço de $C\left([-r, 0], \mathbb{R}^{n}\right)$. Aplicando tal projeção a solução $W_{t}$, da equação linear não-homogênea da forma (3.11), essa equação se reduz a uma equivalente equação diferencial ordinária linear autônoma, com um termo forçante não homogêneo, relativo a $\rho(t)$. A prova do lema segue essa linha.

Desse modo, o próximo lema é essencialmente um resultado de pertubação linear, o qual descreve o comportamento das soluçôes das equações de camada de transição (3.4) nas variedades estável e instável do zero.

É um caso particular do Lema 4.2 de [1] e será útil para o Teorema 4.1.

Lema 3.1 Assumamos que $F=\left(f_{1}, f_{2}\right)$ satisfaz as hipóteses (H.1) - (H.3) e (H.5), com $f_{1}, f_{2}: \mathbb{R} \rightarrow \mathbb{R}$, diferenciáveis até ordem 2 na origem e impares. Suponhamos que

$$
y_{1+}, y_{2+}:[-r, \infty) \rightarrow \mathbb{R}
$$

são funções $C^{1}$ satisfazendo as equações de camada de transição (3.4) para todo $t \geq 0$, e que, para o mesmo $r \geq 0$,

$$
y_{1-}, y_{2-}:[-\infty, 0) \rightarrow \mathbb{R}
$$

são funções $C^{1}$ satisfazendo as equações de camada de transição (3.4) para todo $t \leq 0$. Suponhamos ainda, que existam constantes $T_{+} \geq 0$ e $T_{-} \geq 0$, tais que 


$$
\left\{\begin{array}{l}
y_{1+}(t) \leq 0, \dot{y}_{1+}(t) \geq 0, \quad \text { e } y_{2+}(t) \geq 0, \text { se } t \geq T_{+} \\
y_{1-}(t) \geq 0, \dot{y}_{1-}(t) \geq 0, \text { e } y_{2-}(t) \leq 0, \text { se } t \leq-T_{-} \\
\lim _{t \rightarrow \infty}\left(y_{1+}(t), y_{2+}(t)\right)=\lim _{t \rightarrow-\infty}\left(y_{1-}(t), y_{2-}(t)\right)=(0,0)
\end{array}\right.
$$

Então, ou $\left(y_{1+}(t), y_{2+}(t)\right)=(0,0)$ para todo $t \geq-r$ ou $\left(y_{1-}(t), y_{2-}(t)\right)=(0,0)$ para todo $t \leq 0$.

Prova: A prova do Lema 3.1 é análoga a do Lema 4.2 de [1].

Observação 3.3 As hipóteses do Lema 3.1 e o fato de $f_{2}$ ser monótona decrescente num intervalo contendo a origem implicam na monotonicidade de $y_{2+}(t)$ e $y_{2-}(t)$, para $t \geq T_{+}$e $t \leq-T_{-}$, respectivamente, conforme Figura 3.1. A prova desse fato é apresentada, com ligeiras adaptações, em [1 (observação 4.1)].

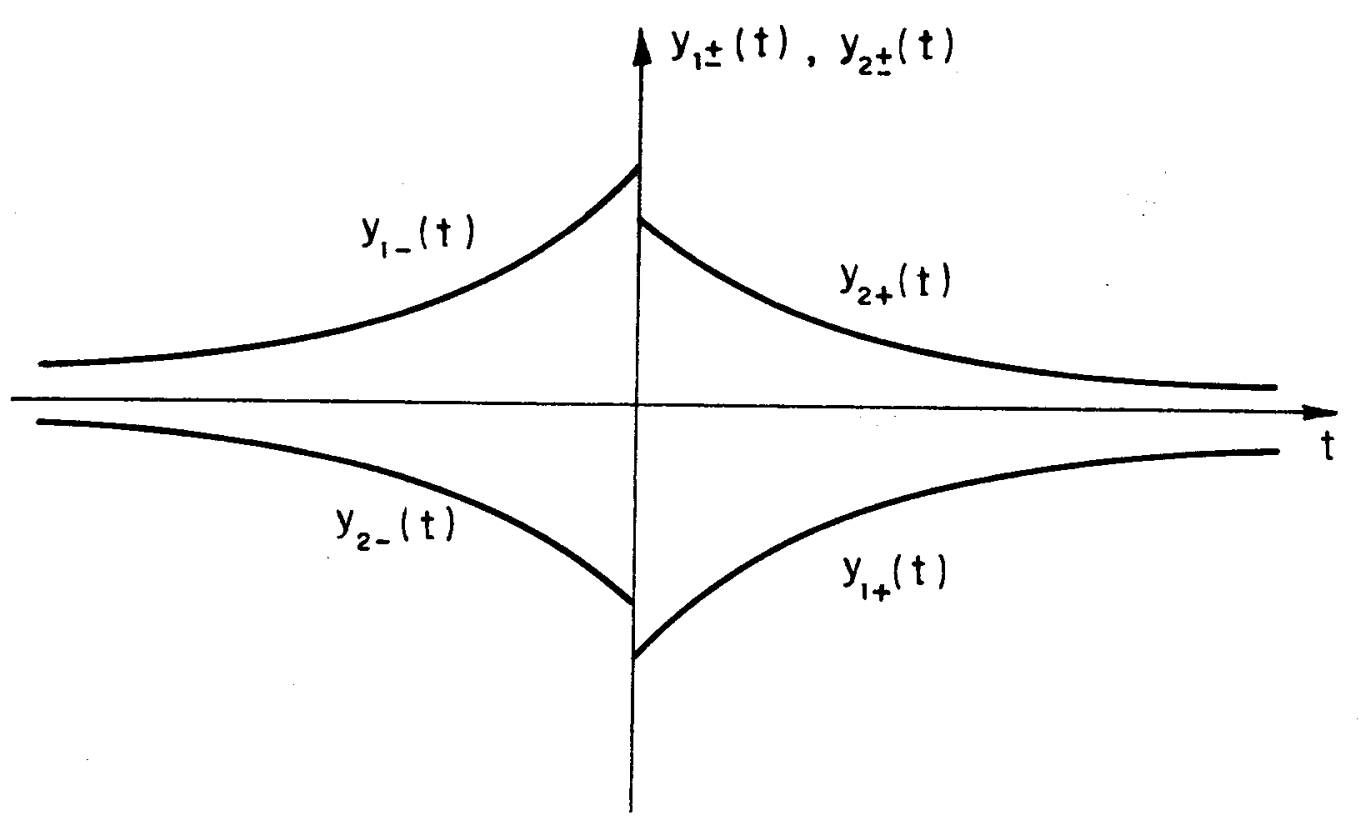

Figura 3.1 


\section{Capítulo 4}

\section{Forma assintótica das soluções periódicas quando $\varepsilon \rightarrow 0_{+}$}

Neste capítulo, vamos estudar a forma assintótica das soluções $x_{\varepsilon}(t)$ de $(2.1) \varepsilon$ quando $\varepsilon \rightarrow 0_{+}$, para $F=\left(f_{1}, f_{2}\right)$, satisfazendo as hipóteses (H.1) - (H.3) com $f_{1}, f_{2}: \mathbb{R} \rightarrow \mathbb{R}$, diferenciáveis até ordem 2 na origem e ímpares. Vamos nos ater ao caso particular de existir uma constante positiva $A \leq M$, tal que, $f_{1}=-f_{2}=f$ em $[-A, A]$ e $f([-A, A]) \subseteq[-A, A]$ e as $S^{*}$-soluções com condições iniciais em $C([-1,0], Q)$, onde $Q$ é dado na Observação 2.3 .

Em alguns momentos vamos assumir a seguinte hipótese:

(H.6) Existe uma constante positiva $a \leq A$, tal que, se $x_{0} \in(0, A]$ e $x_{n}=f^{n}\left(x_{0}\right)$ é a n-ésima iterada de $f$ em $x_{0}$, para cada $n \geq 0$, então $a=\lim _{n \rightarrow \infty} x_{n}$.

Consideramos dois casos.

\section{Caso I}

$$
f_{1}=-f_{2}=f \text { monótona em }[-A, A] \text { e }|f f(x)|>|x|, x \in(-A, A) \text { e } x \neq 0 .
$$
Nesse caso o ponto $A$ é 2 periódico para a funçâo $f$. 


\section{Caso II}

$f_{1}=-f_{2}=f$ satisfaz a hipótese adicional (H.6). Nesse caso o ponto $a \leq A$ é 2 periódico para a função $f$.

Se $a \in \mathbb{R}$ é dado em (H.6), seja a função descontínua $o q(t)=\left(o q_{1}(t), o q_{2}(t)\right)$ dada por:

$$
o q_{1}(t)=\left\{\begin{aligned}
a, & \text { se } 4 n-1<t<4 n+1 \\
-a, & \text { se } 4 n+1<t<4 n+3, \quad n \in \mathbb{Z}
\end{aligned}\right.
$$

e $o q_{2}(t)=o q_{1}(t+1), t \in \mathbb{R}$.

Veja a Figura 4.1 .

Quando $a=A$, denotamos $o q=o \bar{q}=\left(o \bar{q}_{1}, o \bar{q}_{2}\right)$.

Nessas condições o sistema limite

$$
\left\{\begin{array}{l}
x_{1}(t)=f\left(x_{2}(t-1)\right) \\
x_{2}(t)=-f\left(x_{1}(t-1)\right)
\end{array}\right.
$$

tem uma solução descontínua $o \bar{q}(t)$ no primeiro caso e $o q(t)$ no segundo caso.

Nosso objetivo é mostrar que a função $o q(t)$ descreve o comportamento assintótico de $x_{\varepsilon}(t)$ quando $\varepsilon \rightarrow 0_{+}$. Mais precisamente, mostraremos que $x_{\varepsilon}(t) \rightarrow o q(t)$ nas partes compactas de $\mathbb{R}-\mathbb{Z}$.

Em vizinhanças de amplitude $\mathcal{O}(\varepsilon)$, dos pontos inteiros $t=-1,0,1$ e 2, estudaremos o comportamento de $x_{\varepsilon}(t)$ através de uma solução das equações de camada de transição.

No segundo caso, perto dos inteiros, onde a função $o q(t)$ é descontínua, o comportamento de $x_{\varepsilon}(t)$, quando $\varepsilon \rightarrow 0_{+}$, pode ser um tanto complicado. Pode ocorrer um fenômeno similar ao fenômeno de Gibbs da série de Fourier, nessas 
$\mathcal{O}(\varepsilon)$-vizinhança de $t=-1,0,1$ e 2 .

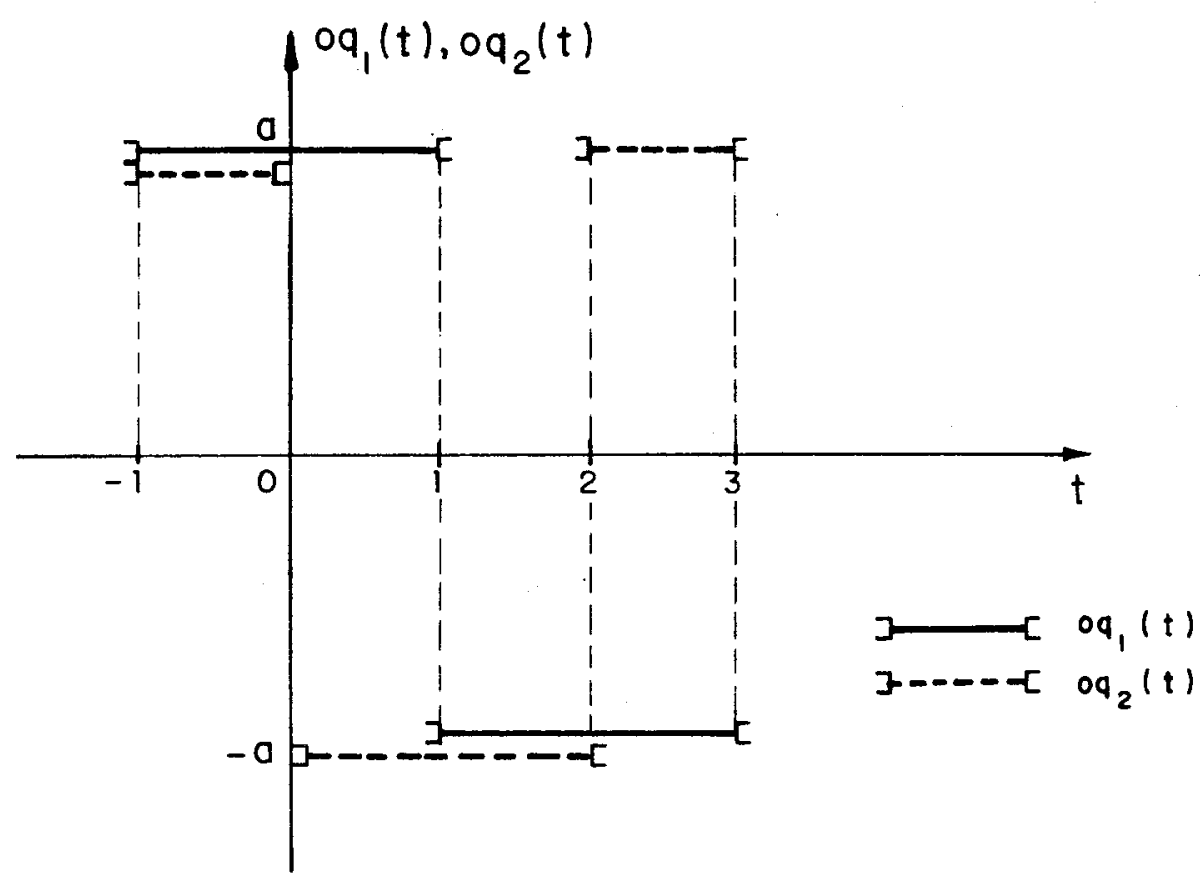

Figura 4.1: Gráfico das coordenadas de uma função $o q(t)$.

Nosso primeiro resultado diz que as normas das $S^{*}$-soluções não se aproximam do zero quando $\varepsilon \rightarrow 0_{+}$.É um caso particular do Lema 4.1 de [1].

Seja $\left\|x_{n}\right\|=\max _{t}\left|x_{n}(t)\right|$.

Lema 4.1 Assumamos que $F=\left(f_{1}, f_{2}\right)$ satisfaça (H.1)-(H.3), com $f_{1}, f_{2}: \mathbb{R} \rightarrow$ $\mathbb{R}$, diferenciáveis até ordem 2 na origem, impares e que exista $A, 0<A \leq M$, tal que, $f_{1}=-f_{2}=f$ em $[-A, A]$ e $f([-A, A]) \subseteq[-A, A]$. Se $x_{n}(t)$ é uma $S^{*}$-soluçấo do sistema $(2.1) \varepsilon_{n}$, entẫo nâa existe sequência $\varepsilon_{n} \rightarrow 0_{+}$, tal que, $\left\|x_{n}\right\| \rightarrow 0, \operatorname{com} n \rightarrow \infty$. 
Prova: Pode ser dada por ligeira adaptação da prova do Lema 4.1 de [1].

O próximo teorema mostra que a solução permanece afastada do zero, uniformemente em $\varepsilon$, quando $\varepsilon_{n} \rightarrow 0_{+}$, nas partes compactas de $\mathbb{R}-\mathbb{Z}$.

Ainda, o teorema a seguir, junto com o Corolário 4.1 e 4.2, descreve a convergência mais delicada de $x_{\varepsilon}(t)=\left(x_{1 \varepsilon}(t), x_{2 \varepsilon}(t)\right)$, solução de $(2.1) \varepsilon$, perto dos inteiros, quando $\varepsilon \rightarrow 0_{+}$. Observe-se que, de acordo com o teorema da estimativa do período, os segundos zeros $q_{2 \varepsilon}$ e $q_{3 \varepsilon}$ de $x_{1 \varepsilon}$ e $x_{2 \varepsilon}$ tendem a 1 e 2 , respectivamente, quando $\varepsilon \rightarrow 0_{+}$. Devido à simetria das $S^{*}$-soluções resultados análogos são obtidos nas vizinhanças de -1 e 0 , em relação aos primeiros zeros de $x_{1 \varepsilon}(t) \mathrm{e}$ $x_{2 \varepsilon}(t)$, respectivamente.

Teorema 4.1 Assumamos que $F=\left(f_{1}, f_{2}\right)$ satisfaça (H.1)-(H.3), com $f_{1}, f_{2}: \mathbb{R} \rightarrow \mathbb{R}$, diferenciáveis até ordem 2 na origem e ímpares, e que exista $A, 0<A \leq M$, tal que, $f_{1}=-f_{2}=f$ em $[-A, A]$ e $f([-A, A]) \subseteq[-A, A]$. Seja $0<c \leq A$ satisfazendo as condiçôes do Teorema 2.1 e $\gamma>1$ satisfazendo a condiçâo (2.6). Ainda, seja $c_{0}>0$ satisfazendo $c_{0}<c$. Então, existe $\varepsilon_{0}>0$, tal que, se $\varepsilon \leq \varepsilon_{0}$ e $x(t)$ é uma $S^{*}$-solução do sistema $(2.1)_{\varepsilon}$ temos

$$
\begin{gathered}
\max _{t} x_{1}(t)>c_{0}, \\
\min _{t} x_{2}(t)<-c_{0} .
\end{gathered}
$$

Ainda, existe uma constante $K>0$, tal que, se $x(t)=\left(x_{1}(t), x_{2}(t)\right)$ é uma $S^{*}$ solução de $(2.1)_{\varepsilon}$, para algum $\varepsilon>0$, então

$$
\begin{gathered}
\left\{t \in\left[b_{1}, c_{1}\right]:-c_{0} \leq x_{1}(t) \leq c_{0}\right\} \subseteq\left[q_{2}-K \varepsilon, q_{2}+K \varepsilon\right], \\
\left\{t \in\left[d_{2}, a_{2}+P\right]:-c_{0} \leq x_{2}(t) \leq c_{0}\right\} \subseteq\left[q_{3}-K \varepsilon, q_{3}+K \varepsilon\right],
\end{gathered}
$$


onde $b_{1}, c_{1}, a_{2} e d_{2}$ estão definidos na propriedade $J$ e $P$ é o periodo mínimo de $x(t)=\left(x_{1}(t), x_{2}(t)\right)$.

As constantes $\varepsilon_{0}$ e $K$ dependem de $c_{0}$, mas nâo da solução $x(t)$; além disso, $K$ não depende de $\varepsilon$.

Prova: Fixemos um número $k$ satisfazendo $c_{0}<k c$ e $0<k<1$.

Se $x(t)=\left(x_{1}(t), x_{2}(t)\right)$ é alguma $S^{*}$-solução do sistema $(2.1)_{\varepsilon}$, definamos quantidades $c_{*}$ dependendo da solução $x(t)$ por

$$
c_{*}=\min \left\{c_{0}, \max _{t} k x_{1}(t)\right\}>0
$$

e, portanto,

$$
-c_{*}=\max \left\{-c_{0}, \min _{t} k x_{2}(t)\right\}<0
$$

Com a finalidade de provarmos o teorema, é suficiente mostrarmos que existem $\varepsilon_{0}>0$ e $K_{0}>0$ que não dependem da solução, tais que, se $\varepsilon \leq \varepsilon_{0}$, então

$$
\begin{gathered}
\left\{t \in\left[b_{1}, c_{1}\right]:-c_{*} \leq x_{1}(t) \leq c_{*}\right\} \subseteq\left[q_{2}-K_{0} \varepsilon, q_{2}+K_{0} \varepsilon\right] \\
\left\{t \in\left[d_{2}, a_{2}+P\right]:-c_{*} \leq x_{2}(t) \leq c_{*}\right\} \subseteq\left[q_{3}-K_{0} \varepsilon, q_{3}+K_{0} \varepsilon\right] \\
\max x_{1}(t)>k^{-1} c_{0} \\
\\
\min x_{2}(t)<-k^{-1} c_{0}
\end{gathered}
$$

sempre que $x(t)=\left(x_{1}(t), x_{2}(t)\right)$ é uma $S^{*}$-solução de $(2.1)_{\varepsilon}$ com os dois segundos zeros, $q_{2}$ e $q_{3}$ de $x_{1}(t)$ e $x_{2}(t)$, respectivamente, $b_{1}, c_{1}, a_{2}$ e $d_{2}$ como na propriedade $J$ e $P$ periodo mínimo de $x(t)$. 
Se (4.9) e (4.10) são válidas, então $c_{*}=c_{0}$ e portanto (4.7) e (4.8) implicam (4.3) e (4.4) $\operatorname{com} K=K_{0}$.

Suponhamos (4.7) e (4.8) não verdadeiras. Entâo existem sequências $\varepsilon_{n} \rightarrow 0_{+}$ e $K_{n} \rightarrow+\infty$, e uma sequência $x_{n}(t)$ de $S^{*}$-soluçôes de $(2.1)_{\varepsilon}$, tais que

$$
\left\{t \in\left[b_{1 n}, c_{1 n}\right]:-c_{n}^{*} \leq x_{1 n}(t) \leq c_{n}^{*}\right\} \nsubseteq\left[q_{2 n}-K_{n} \varepsilon_{n}, q_{2 n}+K_{n} \varepsilon_{n}\right]
$$

ou

$$
\left\{t \in\left[d_{2 n}, a_{2 n}+P_{n}\right]:-c_{n}^{*} \leq x_{2 n}(t) \leq c_{n}^{*}\right\} \nsubseteq\left[q_{3 n}-K_{n} \varepsilon_{n}, q_{3 n}+K_{n} \varepsilon_{n}\right]
$$

onde $c_{n}^{*}$ são dados por (4.5)-(4.6), com $x_{n}(t)=\left(x_{1 n}(t), x_{2 n}(t)\right)$ no lugar de $x(t)=$ $\left(x_{1}(t), x_{2}(t)\right)$.

Suponhamos (4.11) verdadeira, o que implica, para cada $n$

$$
\tau_{1 n}-\sigma_{1 n}>k_{n} \varepsilon_{n}
$$

onde $\tau_{1 n}$ é o menor $t \in\left[q_{2 n}, c_{2 n}\right]$, tal que, $x_{1}(t)=-c_{n}^{*}$ e $\sigma_{1 n}$ é o maior $t \in\left[b_{1 n}, q_{2 n}\right]$, tal que, $x_{1}(t)=c_{n}^{*}, c_{2 n}$ dado na propriedade $J$. Veja a Figura 4.2.

Notemos que $x_{1 n}(t)$ é monótona decrescente em $\left[\sigma_{1 n}, \tau_{1 n}\right] \subseteq\left[b_{1 n}, c_{1 n}\right]$, $c_{2 n} \leq c_{1 n}$.

Agora, sejam $\left(y_{1+}(t), y_{2+}(t), r\right)$ e $\left(y_{1-}(t), y_{2-}(t), r\right)$ soluções das equações de camada de trasição associadas as sequências $\left(\varepsilon_{n}, x_{n}(t), \tau_{1 n}\right)$ e $\left(\varepsilon_{n}, x_{n}(t), \sigma_{1 n}\right)$ respectivamente, como descrito na Proposição 3.1 e na Definição 3.2.

A monotonicidade de $x_{1 n}(t)$ em $\left[\sigma_{1 n}, \tau_{1 n}\right]$, as definiçôes de $\sigma_{1 n}, \tau_{1 n}, y_{1+}(t)$, $y_{1-}(t)$ e a equação (4.13), implicam que

$$
\begin{gathered}
\left\{\begin{array}{l}
-c \leq y_{1+}(t) \leq c, \text { se } t>0, \\
-c \leq y_{1-}(t) \leq c, \text { se } t<0,
\end{array}\right. \\
\left\{\begin{array}{l}
\dot{y}_{1+}(t) \geq 0, \text { se } t \geq 0 \\
\dot{y}_{1-}(t) \geq 0,
\end{array} \text { se } t \leq 0\right.
\end{gathered}
$$

Portanto os limites $\lim _{t \rightarrow+\infty} y_{1 \pm}(t)=L_{+} \quad$ e $\lim _{t \rightarrow-\infty} y_{1 \pm}(t)=L_{-} \quad$ existem, com $L_{+}, L_{-} \in[-c, c] \subseteq[-A, A]$, onde $L_{+}=L$ e $L_{-}=-L$. 
Pela parte e) da Proposição 3.1 vemos que $L_{+}$e $L_{-}$são tais que

$$
\left\{\begin{array}{l}
f_{1} \circ f_{2}\left(L_{+}\right)=L_{-}, \\
f_{2} \circ f_{1}\left(L_{+}\right)=L_{-}, \\
f_{2} \circ f_{1}\left(L_{-}\right)=L_{+}, \\
f_{1} \circ f_{2}\left(L_{-}\right)=L_{+} .
\end{array}\right.
$$

Como $f_{1}=-f_{2}$ em $[-A, A]$ e $\gamma>1$ segue que, (4.16) está apenas satisfeita para $L_{+}=L_{-}=0$.

Logo,

$$
\left\{\begin{aligned}
-c \leq y_{1+}(t) \leq 0, & t \geq 0 \\
0 \leq y_{1-}(t) \leq c, & t \leq 0
\end{aligned}\right.
$$

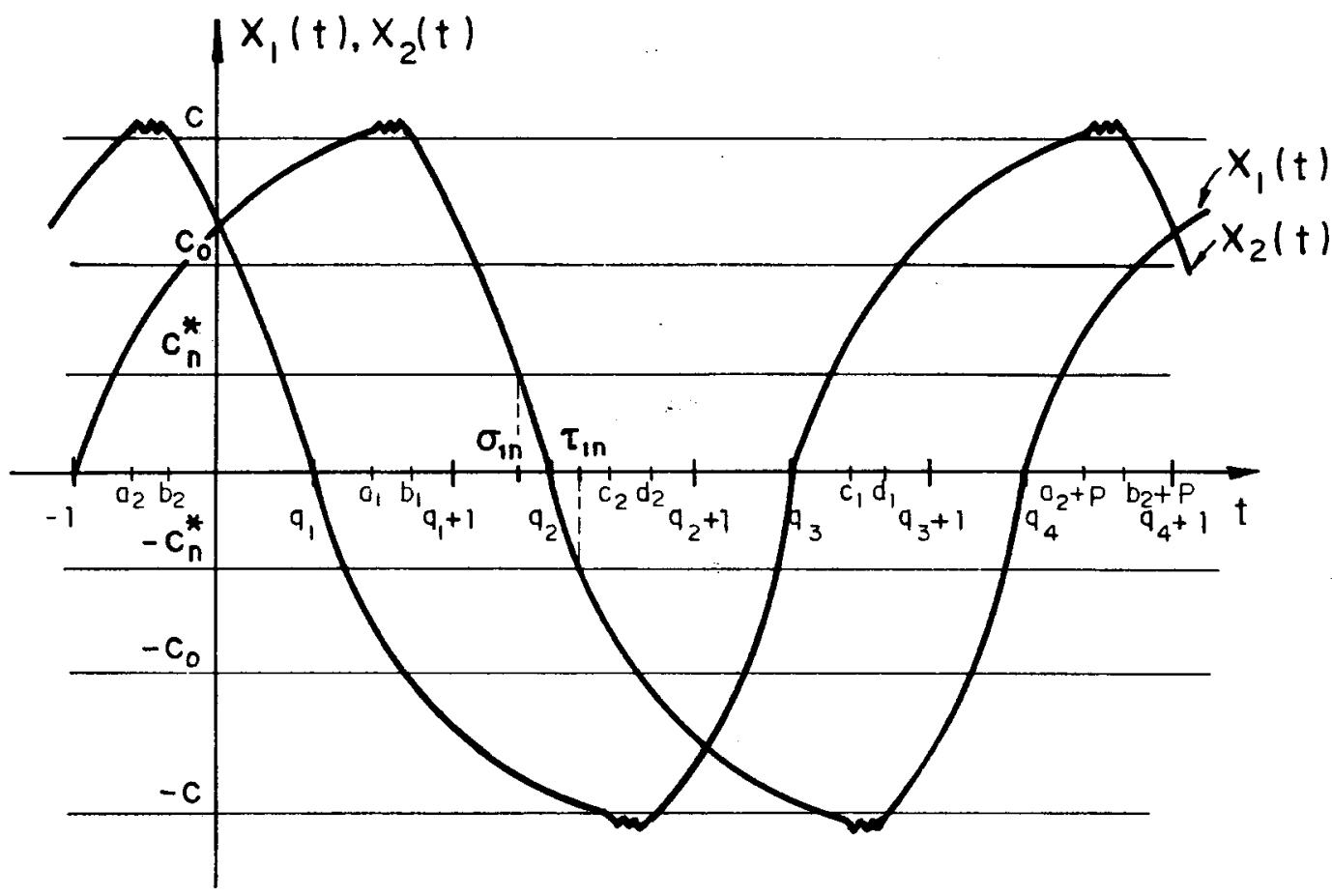

Figura 4.2 
Além disso, partes d) e e) da Proposição 3.1 implicam que existem $T_{+} \geq 0$ e $T_{-} \geq 0$, tais que

$$
\begin{cases}y_{2+}(t) \geq 0, & t \geq T_{+} \\ y_{2-}(t) \leq 0, & t \leq-T_{-}\end{cases}
$$

e, portanto,

$$
\lim _{t \rightarrow+\infty}\left(y_{1+}(t), y_{2+}(t)\right)=\lim _{t \rightarrow-\infty}\left(y_{1-}(t), y_{2-}(t)\right)=(0,0)
$$

Notamos que nem $\left(y_{1+}(t), y_{2+}(t)\right)$ e nem $\left(y_{1-}(t), y_{2-}(t)\right)$ são identicamente nulas; pois por definição temos

$$
\left\{\begin{array}{l}
y_{1+}(0)=\lim _{n \rightarrow \infty} x_{1 n}\left(\tau_{1 n}\right)=\lim _{n \rightarrow \infty}-c_{n}^{*}, \\
y_{1-}(0)=\lim _{n \rightarrow \infty} x_{1 n}\left(\sigma_{1 n}\right)=\lim _{n \rightarrow \infty}+c_{n}^{*},
\end{array}\right.
$$

para alguma subsequência, e por (2.33), (2.34) e Lema 4.1, segue-se que para algum $\Omega>1$, temos

$$
\left\{\begin{array}{l}
\lim _{n \rightarrow \infty} \inf c_{n}^{*} \geq \min \left\{c_{0}, \liminf \frac{k}{\Omega}\left\|x_{n}\right\|\right\}>0, \\
\lim _{n \rightarrow \infty} \sup -c_{n}^{*} \leq \max \left\{-c_{0},-\lim \sup \frac{k}{\Omega}\left\|x_{n}\right\|\right\}<0 .
\end{array}\right.
$$

Portanto, de (4.20) e (4.21), concluimos que

$$
\left\{\begin{array}{l}
y_{1+}(0)<0 \\
y_{1-}(0)>0
\end{array}\right.
$$

Agora estamos aptos a obter uma contradição. Das desigualdades (4.15), $(4.17),(4.18)$ e $(4.19)$, vemos que as soluçôes $\left(y_{1 \pm}(t), y_{2 \pm}(t)\right)$ satisfazem as hipóteses do Lema 3.1. Isso contradiz (4.22). A partir dessa contradição, temos as inclusões (4.7) e (4.8) como desejávamos.

Provemos agora, as desigualdades (4.9) e (4.10) para $\varepsilon$ pequeno.

Suponhamos que pelo menos uma delas não é verdadeira, isto é, para uma sequência $x_{n}(t)$ de $S^{*}$-soluçôes de $(2.1)_{\varepsilon}, \varepsilon_{n} \rightarrow 0_{+}$,

$$
\max x_{1 n}(t) \leq k^{-1} c_{0}<c,
$$


para cada $n$.

Como antes, consideremos os pontos $\sigma_{1 n}$ e $\tau_{1 n}$, tais que, $x_{1 n}\left(\sigma_{1 n}\right)=c n^{*}$ e $x_{1 n}\left(\tau_{1 n}\right)=-c n^{*}$.

Sabemos que

$$
\dot{x}_{1 n}(t) \leq 0, t \in\left[\sigma_{1 n}, \tau_{1 n}\right]
$$

Ainda por (4.7), vemos que

$$
\frac{\tau_{1 n}-\sigma_{1 n}}{\varepsilon_{n}}
$$

é uma sequência limitada.

Seja $\left(y_{1}(t), y_{2}(t), r\right)$ uma solução das equações de camada de transição associada a $\left(\varepsilon_{n}, x_{n}(t), \sigma_{1 n}\right)$.

Da definição de $y_{1}(t)$ e das condições $(4.23),(4.24)$ e (4.25), vemos que

$$
y_{1}\left(q_{2}\right) \leq y_{1}(t)<c ; \text { se } t \geq q_{2}
$$

Usando a primeira desigualdade de (4.21), temos

$$
y_{1}\left(q_{2}\right) \geq \lim _{n \rightarrow \infty} \inf x_{1 n}\left(\sigma_{1 n}\right)=\lim _{n \rightarrow \infty} \inf c_{n}^{*}>0 .
$$

Portanto, de (4.26) e (4.27) temos que $\lim _{t \rightarrow \infty} y_{1}(t)=L$ existe e satisfaz $0<L<c$.

Pela parte e) da Proposição 3.1 o número $L$ é tal que, $f_{1}\left(f_{2}(L)\right)=-L$; mas como já notamos, $f_{1} \circ f_{2}$ não tem valor que satisfaça essa igualdade em $(0, c]$.

Essa contradição completa a prova do teorema.

Corolário 4.1 Assumamos as hipóteses e notaçôes do Teorema 4.1, com relação a função $F=\left(f_{1}, f_{2}\right)$ e os números $A$, c e $\gamma$. Seja $x_{n}(t)$ uma $S^{*}$-solução do sistema $(2.1) \varepsilon_{n}$, para alguma sequência $\varepsilon_{n} \rightarrow 0_{+}$, e definamos $r_{n}>0$ por $p_{n}=$ $2\left(1+\varepsilon_{n} r_{n}\right)$, onde $p_{n}$ é metade do período minimo de $x_{n}(t)=\left(x_{1 n}(t), x_{2 n}(t)\right)$, soluçấo de $(2.1) \varepsilon_{n}$. 
Entâo, tomando uma subsequência, temos

$$
\begin{aligned}
& x_{1 n}\left(q_{2 n}-\varepsilon_{n} t\right) \rightarrow y_{1}(t), \\
& x_{2 n}\left(q_{2 n}+p_{n} / 2-\varepsilon_{n} t\right) \rightarrow y_{2}(t),
\end{aligned}
$$

uniformemente em subconjuntos compactos de $\mathbb{R}$, para funções $C^{1}, y_{1}(t)$ e $y_{2}(t)$. Além disso, temos convergência das derivadas primeiras com respeito a $t \mathrm{em}$ (4.28), e as funções $y_{1}(t)$ e $y_{2}(t)$ satisfazem as equações de camada de transiçâa

$$
\left\{\begin{array}{l}
\dot{y}_{1}(t)=y_{1}(t)+f_{1}\left(y_{2}(t-r)\right) \\
\dot{y}_{2}(t)=y_{2}(t)-f_{2}\left(y_{1}(t-r)\right)
\end{array}\right.
$$

para $t \in \mathbb{R}$, onde

$$
r=\lim _{n \rightarrow \infty} r_{n} \text { é estritamente positivo. }
$$

Ainda, as soluções $y_{1}(t)$ e $y_{2}(t)$ satisfazem as limitações

$$
\left|y_{1}(t)\right|,\left|y_{2}(t)\right| \leq A
$$

e têm as propriedades assintóticas,

$$
\begin{aligned}
& \lim _{t \rightarrow+\infty} \inf y_{1}(t) \geq c \text { e } \lim _{t \rightarrow-\infty} \inf y_{2}(t) \geq c \\
& \lim _{t \rightarrow-\infty} \sup y_{1}(t) \leq-c \text { e } \lim _{t \rightarrow+\infty} \sup y_{2}(t) \leq-c
\end{aligned}
$$

e,

$$
\begin{aligned}
& \lim _{t \rightarrow+\infty} \sup y_{1}(t)>c \text { e } \lim _{t \rightarrow-\infty} \sup y_{2}(t)>c, \\
& \lim _{t \rightarrow-\infty} \inf y_{1}(t)<-c \text { e } \lim _{t \rightarrow+\infty} \inf y_{2}(t)<-c .
\end{aligned}
$$


Finalmente, $y_{1}(t)$ e $y_{2}(t)$ satisfazem as condiçôes de monotonicidade

$$
\begin{aligned}
& -c<y_{1}(t)<c \Rightarrow \dot{y}_{1}(t) \geq 0, \\
& -c<y_{2}(t)<c \Rightarrow \dot{y}_{2}(t) \leq 0 .
\end{aligned}
$$

Prova: Análoga a prova do Corolário 4.1 de [1].

Observação 4.1 Embora nosso problema seja bi-dimensional, o que leva a quatro equações de camada de transição, as hipóteses de simetria fazem com que as equações de camada de transição (4.29) e (4.30), aqui determinadas, sejam as mesmas de [1]. Assim, suas propriedades descritas nas proposições e observações a seguir estão basicamente contidas no Capítulo 4 de [1] e são apresentadas neste trabalho principalmente para tornar a leitura independente.

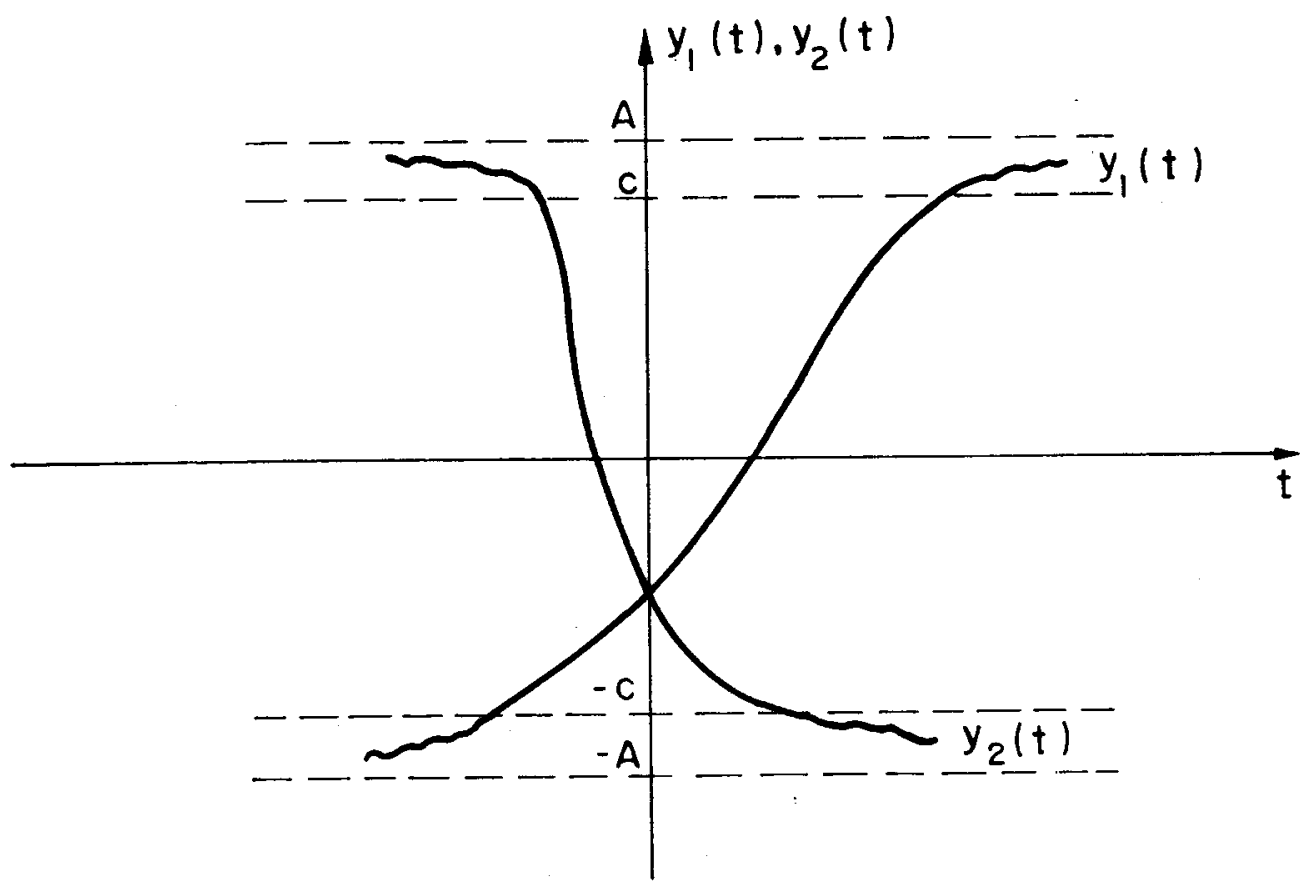

Figura 4.3: Soluçôes das equações de camada de transição do Corolário 4.1. 
Observação 4.2 Observamos que no Corolário 4.1 não afirmamos que os limites (4.28) e (4.31) existem para a sequência $\varepsilon_{n}$, mas somente para alguma subsequência. Se conseguissemos mostrar que, para alguma função $F$, o valor $r>0$ e a solução $\left(y_{1}(t), y_{2}(t)\right)$ das equações da camada de transição é única, satisfazendo as condiçôes (4.32), (4.33), (4.34) e (4.35), então os limites (4.28) e (4.31) existiriam para a sequência $\varepsilon_{n}$.

O corolário a seguir refina os resultados do Teorema 4.1.

Corolário 4.2 Assumamos as hipóteses e notações do Teorema 4.1, com relaçãa a função $F=\left(f_{1}, f_{2}\right)$ e os números $A, c \in \gamma$.

Ainda, seja $I=[-\alpha, \alpha], 0<\alpha \leq A, 0 \in I$, tal que.

$$
\begin{aligned}
& f_{1}^{\prime}(x)>0, \quad x \in I \\
& f_{2}^{\prime}(x)<0, \quad x \in I
\end{aligned}
$$

e $J$ um intervalo compacto; tal que

$$
0 \in J \subseteq(-c, c) \cap f(I) \cap f f(I)
$$

Então, existem constantes positivas $\varepsilon_{0}$ e $K$, tais que, se $x_{\varepsilon}(t)$ é uma $S^{*}$-solução de $(2.1)_{\varepsilon}$ para algum $\varepsilon \leq \varepsilon_{0}$, temos que

$$
x_{1}(t), x_{2}(t) \in J \Rightarrow\left|\dot{x}_{1}(t)\right|,\left|\dot{x}_{2}(t)\right| \geq \frac{1}{K \varepsilon}
$$

Prova: Análoga a do Corolário 4.2 de [1].

O próximo corolário descreve as soluções das equaçôes de camada de transição obtidas no Corolário 4.1 sob a hipótese adicional de monotonicidade para as coordenadas de $F=\left(f_{1}, f_{2}\right)$. 
Corolário 4.3 Assumamos que $F=\left(f_{1}, f_{2}\right)$ satisfaça as hipóteses (H.1) - (H.3), $\operatorname{com} f_{1}, f_{2}: \mathbb{R} \rightarrow \mathbb{R}$, diferenciáveis até ordem 2 na origem e ímpares, e que exista $A, 0<A \leq M$, tal que, $f_{1}=-f_{2}=f$ é monótona em $[-A, A]$ e $f([-A, A]) \subseteq$ $[-A, A]$. Ainda, $|f f(x)|>|x|$ sempre que $x \in(-A, A)$ e $x \neq 0$.

Seja $\left(y_{1}(t), y_{2}(t)\right)$, e r como no Corolário 4.1.

Então $y_{1}(t)$ e $y_{2}(t)$ satisfazem as condiçôes de monotonicidade

$$
\dot{y}_{1}(t) \geq 0 \text { e } \dot{y}_{2}(t) \leq 0, \text { para todo } t
$$

e existem os limites

$$
\begin{aligned}
& \lim _{t \rightarrow-\infty}\left(y_{1}(t), y_{2}(t)\right)=(-A, A), \\
& \lim _{t \rightarrow+\infty}\left(y_{1}(t), y_{2}(t)\right)=(A,-A) .
\end{aligned}
$$

Assim, $\left(y_{1}(t), y_{2}(t)\right)$ é uma órbita heteroclínica ligando os pontos estacionários $(-A, A)$ e $(A,-A)$ das equações de camada de transição (4.29) e (4.30).

Prova: Análoga a do Corolário 4.3 de [1].

Observação 4.3 Sob as hipóteses do Corolário 4.3 vemos em que sentido as $S^{*}$-soluções de $(2.1) \varepsilon$, se aproximam, quando $\varepsilon \rightarrow 0_{+}$, da função descontínua $o \bar{q}(t)=\left(o \bar{q}_{1}(t), o \bar{q}_{2}(t)\right)$, dada por:

$$
\begin{aligned}
& o \bar{q}_{1}(t)=\left\{\begin{array}{rll}
A, & \text { se } & 4 n-1<t<4 n+1 \\
-A, & \text { se } & 4 n+1<t<4 n+3
\end{array}\right. \\
& o \bar{q}_{2}(t)=\left\{\begin{array}{rll}
A, & \text { se } & 4 n-1<t<4 n \\
-A, & \text { se } & 4 n<t<4 n+2 \\
A, & \text { se } & 4 n+2<t<4 n+3, \quad n \in \mathbb{Z},
\end{array}\right.
\end{aligned}
$$

$t \in \mathbb{R}, 0<A \leq M$. 
Veja a Figura 4.4

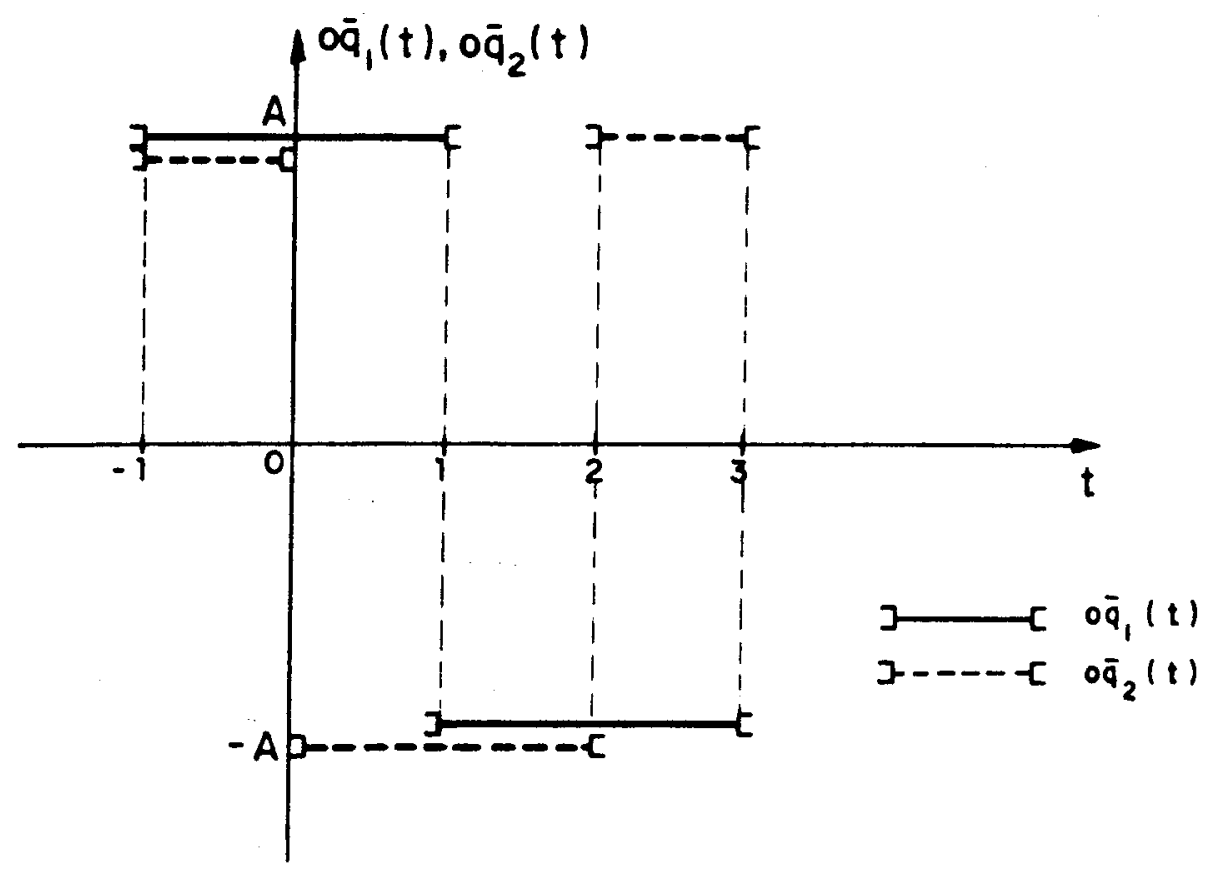

Figura 4.4: As coordenadas de uma função $o \bar{q}(t)=\left(o \bar{q}_{1}(t), o \bar{q}_{2}(t)\right)$.

Realmente as coordenadas de $x(t)=\left(x_{1}(t), x_{2}(t)\right)$ têm a forma simples mostrada na Figura 4.5, quando $\varepsilon$ é pequeno.

Isso segue da simetria das $S^{*}$-soluções $x(t)$ de $(2.1)_{\varepsilon}$, e do fato que para qualquer constante positiva $c \leq A$, a soluçâo $x(t)$ satisfaz propriedade $J$ entre $-c$ e $c$, devido à monotonicidade das funçôes coordenadas de $F$.

Antes de enunciarmos o Corolário 4.4 que, sob a hipótese adicional (H.6), descreve as soluções das equações de camada de transição, obtidas no Corolário 4.1, daremos um lema técnico, Lema 4.1, sobre essas soluçôes. Trata-se do Lema 3.3 de [1], que será útil para o Corolário 4.4 e o Teorema 4.2.

Ainda, é importante observar novamente, que os resultados aqui obtidos são para as soluções das equaçôes de camada de transição dadas no Corolário 4.1 e, 
portanto, considerando-se os segundos zeros das coordenadas de $x_{\varepsilon}(t), S^{*}$-soluçào de $(2.1)_{\varepsilon}$. Devido à simetria de $x_{\varepsilon}(t)$, resultados análogos valem para as $O(\varepsilon)$ vizinhanças dos primeiros zeros das coordenadas de $x_{\varepsilon}(t)$.

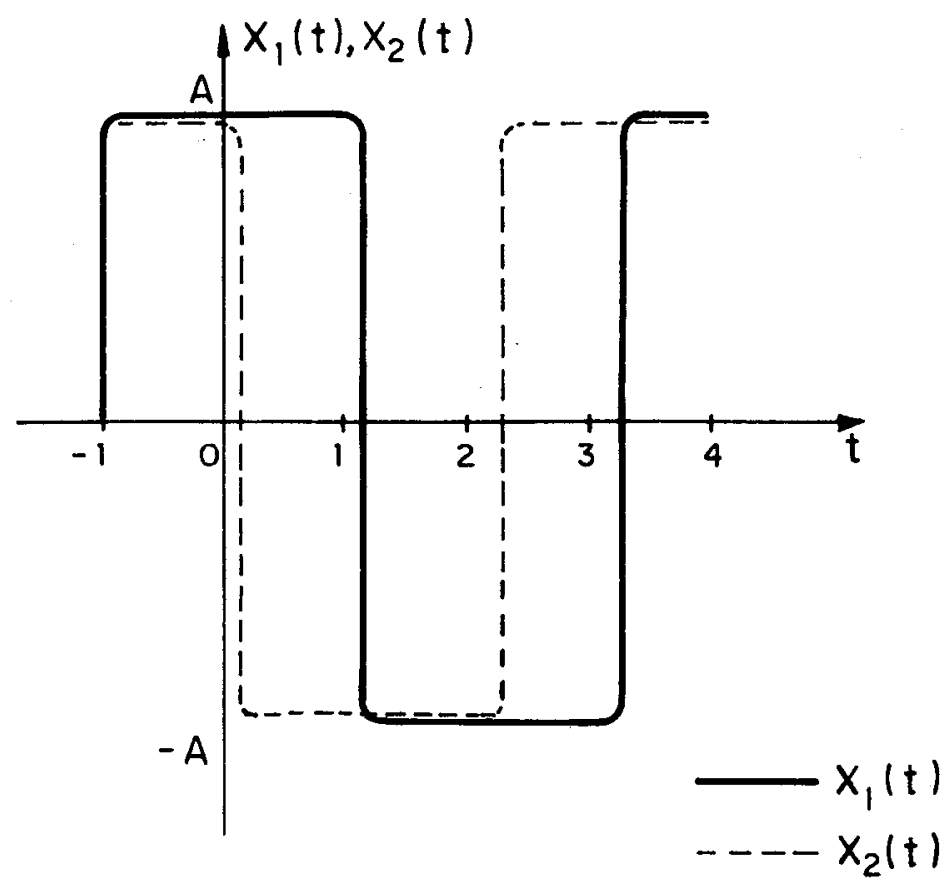

Figura 4.5

Lema 4.2 Assumamos que $F=\left(f_{1}, f_{2}\right)$ satisfaça $(H .1)-(H .3)$, com $f_{1}, f_{2}: \mathbb{R} \rightarrow$ $\mathbb{R}$, diferenciáveis até ordem 2 na origem e impares, e que exista $A, 0<A \leq M$, tal que, $f_{1}=-f_{2}=f$ em $[-A, A]$ e $f([-A, A]) \subseteq[-A, A]$. Ainda, suponhamos que $F$ satisfaça (H.6). Sejam $r>0, y_{1}(t)$ e $y_{2}(t)$ soluçôes das equações de camada de transiçâo para $t \in \mathbb{R}$. Suponhamos que, para algum $c>0$, essas soluções satisfaçam

$$
\begin{aligned}
-A & \leq y_{1}(t) \leq-c \\
c & \leq y_{2}(t) \leq A
\end{aligned}
$$

para todo $t \in \mathbb{R}$. 
Então $y_{1}(t)=-a$ e $y_{2}(t)=a$, para todo $t \in \mathbb{R}$, onde a é dado $\mathrm{cm}$ (H.6).

Prova: Ver Lema 3.3 de [1].

Corolário 4.4 Assumamos que $F=\left(f_{1}, f_{2}\right)$ satisfaça (H.1) - (H.3), com $f_{1}, f_{2}$ : $\mathbb{R} \rightarrow \mathbb{R}$, diferenciáveis até ordem 2 na origem e impares, e que exista $A$, $0<A \leq M$, tal que, $f_{1}=-f_{2}=f$ em $[-A, A]$ e $f([-A, A]) \subseteq[-A, A]$. Ainda, suponhamos que $F$ satisfaça (H.6). Sejam $y_{1}(t), y_{2}(t)$ e $r$ como no Corolário 4.1. Então

$$
\begin{aligned}
& \lim _{t \rightarrow-\infty}\left(y_{1}(t), y_{2}(t)\right)=(-a, a), \\
& \lim _{t \rightarrow+\infty}\left(y_{1}(t), y_{2}(t)\right)=(a,-a),
\end{aligned}
$$

com a dado em (H.6).

Portanto, $\left(y_{1}(t), y_{2}(t)\right)$ é uma órbita heteroclínica ligando os pontos estacionários $(-a, a)$ e $(a,-a)$ das equações de camada de transição (4.29) e (4.30).'

Prova: Usando Lema 4.2 a prova é análoga à do Corolário 4.4 de [1].

Observação 4.4 A existência desses limites não dão informações sobre como uma solução de $(2.1) \varepsilon$ comporta-se fora das camadas de transição quando $\varepsilon \rightarrow 0_{+}$. Sem a hipótese da monotonicidade sob $f_{1}, f_{2}$, os resultados até aqui não garantem que, quando $\varepsilon \rightarrow 0_{+}, x(t)=\left(x_{1}(t), x_{2}(t)\right)$, solução de $(2.1)_{\varepsilon}$, está uniformemente próxima nos subconjuntos compactos de $\mathbb{R}-\mathbb{Z}$, da função $o q(t)=\left(o q_{1}(t), o q_{2}(t)\right)$, dada por:

$$
\begin{aligned}
& o q_{1}(t)=\left\{\begin{array}{rll}
a, & \text { se } & 4 n-1<t<4 n+1 \\
-a, & \text { se } & 4 n+1<t<4 n+3,
\end{array}\right. \\
& \boldsymbol{o q}_{2}(t)=\left\{\begin{array}{rll}
a, & \text { se } & 4 n-1<t<4 n \\
-a, & \text { se } & 4 n<t<4 n+3 \\
a, & \text { se } & 4 n+2<t<4 n+3, \quad n \in \mathbb{Z},
\end{array}\right.
\end{aligned}
$$

$t \in \mathbb{R}$. 
Isso será assegurado no Teorema 4.2 , a seguir.

Teorema 4.2 Assumamos que $F=\left(f_{1}, f_{2}\right)$ satisfaşa as hipóteses (H.1) - (H.3), com $f_{1}, f_{2}: \mathbb{R} \rightarrow \mathbb{R}$, diferenciáveis até ordem 2 , impares e que exista $A$, $0<A \leq M$, tal que, $f_{1}=-f_{2}=f$ em $[-A, A]$ e $f([-A, A]) \subseteq[-A, A]$. Ainda suponhamos que $F$ satisfaça (H.6). Sejam $a_{1}, \alpha_{2}$ números positivos tais que, $\alpha_{1}<a<\alpha_{2}$ e $-\alpha_{2}<-a<-\alpha_{1}$, onde a é dado em (H.6). Então existe um número $K>0$ tal que, se $x(t)$ é uma $S^{*}$-solução de $(2.1) \varepsilon$ para algum $\varepsilon$ positivo, então

$$
\begin{array}{r}
\begin{array}{r}
\left\{t \in\left[-1, q_{4}\right] ; x_{1}(t) \notin\left(-\alpha_{2},-\alpha_{1}\right) \cup\left(\alpha_{2}, \alpha_{1}\right)\right\} \subseteq \\
{[-1,-1+K \varepsilon] \cup\left[q_{2}-K \varepsilon, q_{2}+K \varepsilon\right] \cup\left[q_{4}-K \varepsilon, q_{4}\right],}
\end{array} \\
\left\{t \in\left[-1, q_{4}\right] ; x_{2}(t) \notin\left(-\alpha_{2},-\alpha_{1}\right) \cup\left(\alpha_{1}, \alpha_{2}\right)\right\} \subseteq \\
{\left[q_{1}-K \varepsilon, q_{1}+K \varepsilon\right] \cup\left[q_{3}-K \varepsilon, q_{3}+K \varepsilon\right],}
\end{array}
$$

onde $q_{1}, q_{3}$ e $q_{2}, q_{4}$ sâo os primeiros zeros de $x_{2}(t) \in x_{1}(t)$, respectivamente.

Prova: Análoga a prova do Teorema 4.2 de [1].

Observação 4.5 O Corolário 4.4 e o Teorema 4.2, dão uma idéia precisa da convergência das $S^{*}$-soluções $x(t)$ de $(2.1)_{\varepsilon}$, para a função $o q(t)=\left(o q_{1}(t), o q_{2}(t)\right)$, quando $\varepsilon \rightarrow 0_{+}$.

No Corolário 4.3 , onde $f_{1}, f_{2}$ são funções monótonas, as soluções $y_{1}(t)$ e $y_{2}(t)$ das equações de camada de transição são também funções monótonas de $t$. Aqui, é possível que $y_{1}(t)$ ou $y_{2}(t)$ não sejam funções monótonas de $t$. A imagem de $y_{1}(t)$ ou $y_{2}(t)$ pode conter propriamente o intervalo $[-a, a]$.

Se isso ocorrer, então o Corolário 4.4 e o Teorema 4.2 implicam, um tipo peculiar de convergência não uniforme de $x(t)$ para a função $o q(t)$, quando $\varepsilon \rightarrow 0_{+}$, semelhante ao fenômeno de Gibbs da série de Fourier. 
Vamos exemplificar. Suponhamos que

$$
\inf _{t} y_{2}(t)=-a_{*}<-a
$$

para algum número $a_{*}$. Consideremos uma sequência $x_{n}(t)$ de $S^{*}$-soluções de $(2.1) \varepsilon_{n}$, quando $\varepsilon_{n} \rightarrow 0_{+}$. Então, $y_{2}(t)$ é dado pelo

$$
\lim _{n \rightarrow \infty} x_{2 n}\left(q_{2 n}+p_{n} / 2-\varepsilon_{n} t\right)
$$

como no Corolário 4.1.

Assumamos as hipóteses e notações do Teorema 4.2, com relação à função $F=\left(f_{1}, f_{2}\right)$. Então, $x_{2 n}(t)$ converge uniformemente para o valor $a$ em todo subconjunto compacto de $(2,3)$, e uniformente para o valor $-a$ em todo subconjunto compacto de $(1,2)$.

Contudo, perto do ponto de transição $t=2, x_{2}(t)$ passará do valor $-a$ por uma quantidade que não se torna pequena $\operatorname{com} \varepsilon_{n} \rightarrow 0_{+}$. Isto é, do fato $\inf _{t} y_{2}(t)=$ $-a_{*}<-a$ e da definição de $y_{2}(t)$, temos, para $0<\delta<2$ :

$$
\lim \min _{|t-2| \leq \delta} x_{2 n}(t)=-a_{*}<-a
$$

Do mesmo modo, se $\sup _{t} y_{1}(t)=a_{*}>a$ e $0<\delta<2$,

$$
\lim \max _{|t-1| \leq \delta} x_{1 n}(t)=a_{*}>a
$$

Resultados análogos são obtidos perto dos pontos de transição $t=-1$ e $t=0$.

Veja a Figura 4.6 . 

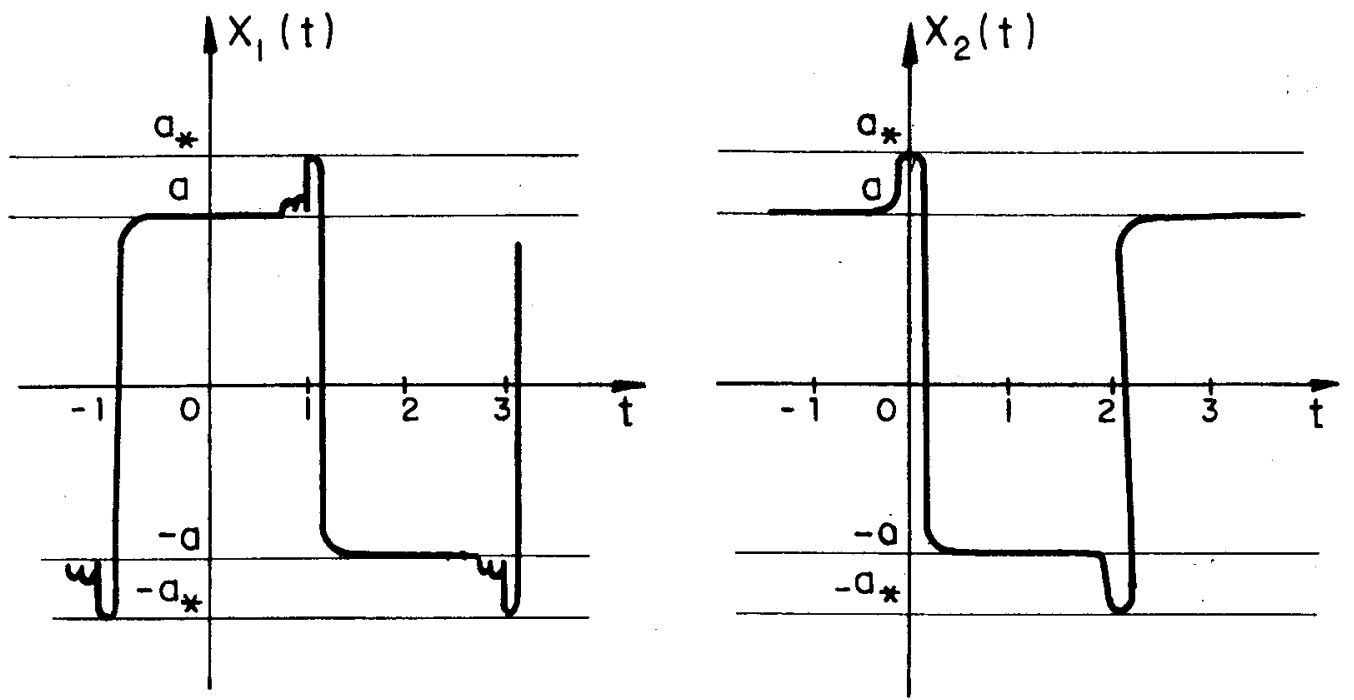

Figura 4.6: Gráficos das Cooordenadas de uma $S^{*}$-solução da Observação 4.5, exibindo o fenômeno de Gibbs.

O próximo resultado dá uma condição suficiente sobre $F=\left(f_{1}, f_{2}\right)$ para esses fenômenos de Gibbs ocorrerem.

Proposição 4.1 Assumamos que $F=\left(f_{1}, f_{2}\right)$ satisfaça as hipóteses (H.1) (H.3), com $f_{1}, f_{2}: \mathbb{R} \rightarrow \mathbb{R}$, diferenciáveis até ordem 2 , impares e que exista $A$, $0<A \leq M$, tal que, $f_{1}=-f_{2}=f$ em $[-A, A]$ e $f([-A, A]) \subseteq[-A, A]$. Ainda, suponhamos que $F$ satisfaça (H.6) e que existe um número positivo $a_{0}<a$, com a dado em (H.6), tal que

$$
a_{0} \leq x<a \Rightarrow f_{2}(x)<-a \quad \text { e } \quad f_{1}(x)>a
$$


Sejam $y_{1}(t), y_{2}(t)$, e r como no Corolário 4.1.

\section{Então}

$$
\sup _{t} y_{1}(t)>a
$$

$o u$

$$
\inf _{t} y_{2}(t)<-a
$$

de modo que, o fenômeno de Gibbs (4.44) ou (4.43) descrito na Observaçâo 4.5 ocorre.

Prova: Análoga a da Proposição 4.1 de [1]. 


\section{Observações finais}

Consideremos o sistema de equações diferenciais com retardamento $(2.1) \varepsilon$.

Nosso trabalho consiste principalmente em estudar o comportamento assintótico de soluções periódicas não triviais de $(2.1)_{\varepsilon}$, quando $\varepsilon \rightarrow 0_{+}$.

Além das hipóteses regularmente assumidas, se $f_{1}=-f_{2}$ em uma vizinhança da origem, então as $S^{*}$-soluções de $(2.1)_{\varepsilon}$ têm período próximo de 4 , quando $\varepsilon \rightarrow 0_{+}$. É válida a seguinte estimativa: $4<P<4(1+\bar{C} \varepsilon)$, onde $P$ é o período das $S^{*}$-soluções de $(2.1)_{\varepsilon}$ e $\bar{C}$ uma constante positiva.

Usando essa estimativa, no contexto do capítulo 4, obtemos o sistema de equações de camada de transição e no Teorema 4.1 e Corolários 4.1 e 4.3 provamos que:

(I) Se $F=\left(f_{1}, f_{2}\right)$ satisfaz as hipóteses (H.1) - (H.3), com $f_{1}, f_{2}: \mathbb{R} \rightarrow \mathbb{R}$, diferenciáveis até ordem 2 na origem, ímpares e se existe $A, 0<A \leq M$, tal que, $f_{1}=-f_{2}=f$ é monótona em $[-A, A], f([-A, A]) \subseteq[-A, A] \mathrm{e}$ ainda, $|f f(x)|>|x|$ sempre que $x \in(-A, A)$ e $x \neq 0$ então, toda $S^{*}$-solução $x(t)=\left(x_{1}(t), x_{2}(t)\right)$ de $(2.1) \varepsilon$ converge uniformemente em subconjuntos compactos de $\mathbb{R}-\mathbb{Z}$, quando $\varepsilon \rightarrow 0_{+}$, para a função $o \bar{q}(t)$, dada em (4.39).

A Figura I ilustra uma $S^{*}$-solução $x(t)=\left(x_{1}(t), x_{2}(t)\right)$ de $(2.1) \varepsilon$, quando $\varepsilon \rightarrow 0_{+}$, nesse caso.

No Teorema 4.1, Corolários 4.1 e 4.4 e Teorema 4.2, provamos que:

(II) Se $F=\left(f_{1}, f_{2}\right)$ satisfaz as hipóteses (H.1) - (H.3), com $f_{1}, f_{2}: \mathbb{R} \rightarrow \mathbb{R}$, diferenciáveis até ordem 2 na origem, ímpares e se existe $A, 0<A \leq M$, tal que, $f_{1}=-f_{2}=f$ em $[-A, A], f([-A, A]) \subseteq[-A, A]$ e ainda, $F$ satisfaz a hipótese (H.6) então, toda $S^{*}$-solução $x(t)=\left(x_{1}(t), x_{2}(t)\right)$ de $(2.1) \varepsilon$ converge uniformemente em subconjuntos compactos de $\mathbb{R}-\mathbb{Z}$, quando $\varepsilon \rightarrow 0_{+}$, para a função $o q(t)$, dada em (4.42). 
Nesse caso, é possível ocorrer um tipo peculiar de convergência não uniforme de $x(t)$ para a funçào $o q(t)$, quando $\varepsilon \rightarrow 0_{+}$, semelhante ao fenômeno de Gibbs da série de Fourier, como exemplificamos na Observação 4.5. Esse fenômeno foi detectado por Mallet-Paret e Nusbaum em [1] no caso escalar e o transportamos aqui para equações bi-dimensionais.

A Figura II ilustra uma $S^{*}$-solução, $x(t)=\left(x_{1}(t), x_{2}(t)\right)$ de $(2.1) \varepsilon$, quando $\varepsilon \rightarrow 0_{+}$, para o caso da Observaçào 4.5 .

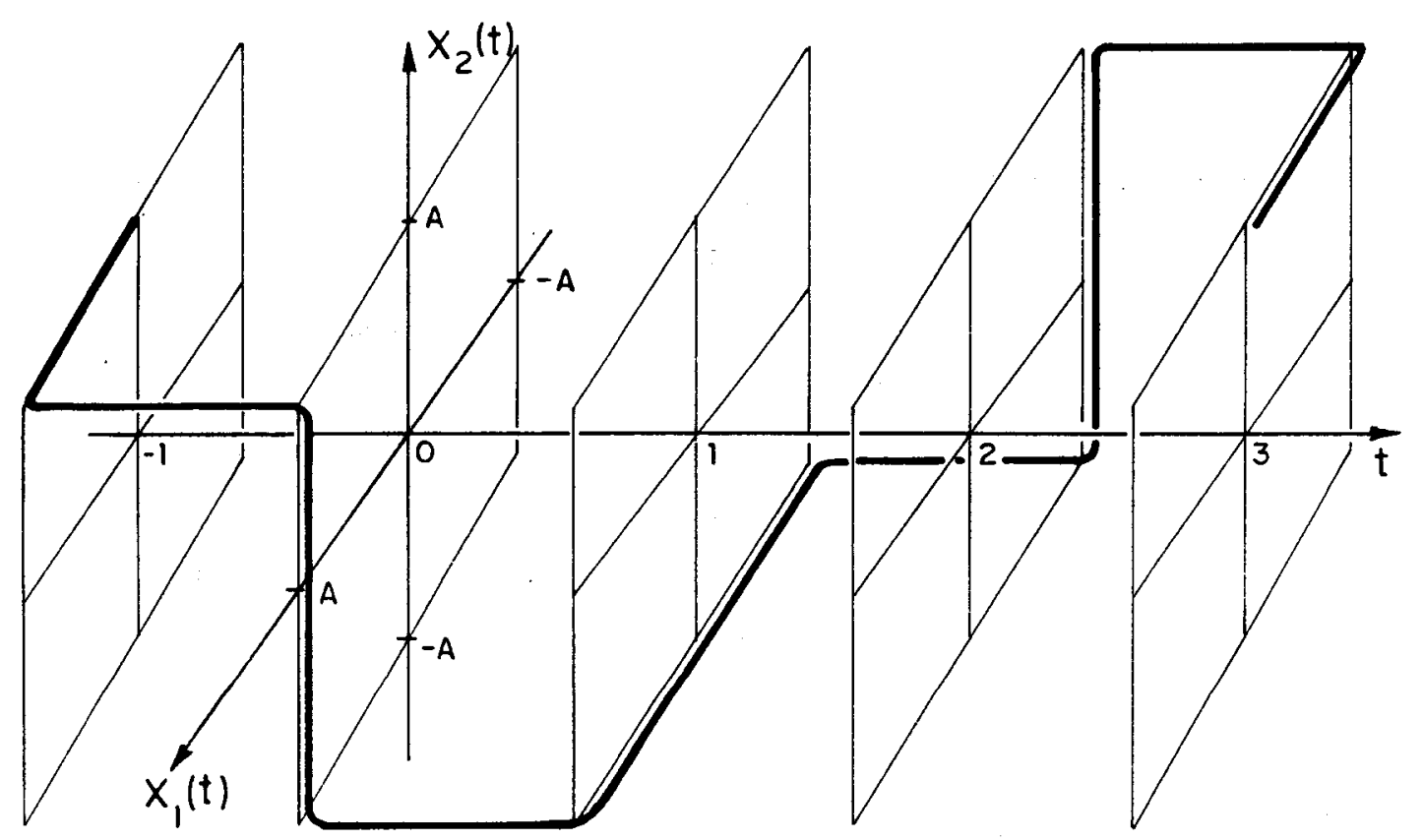

Figura I 


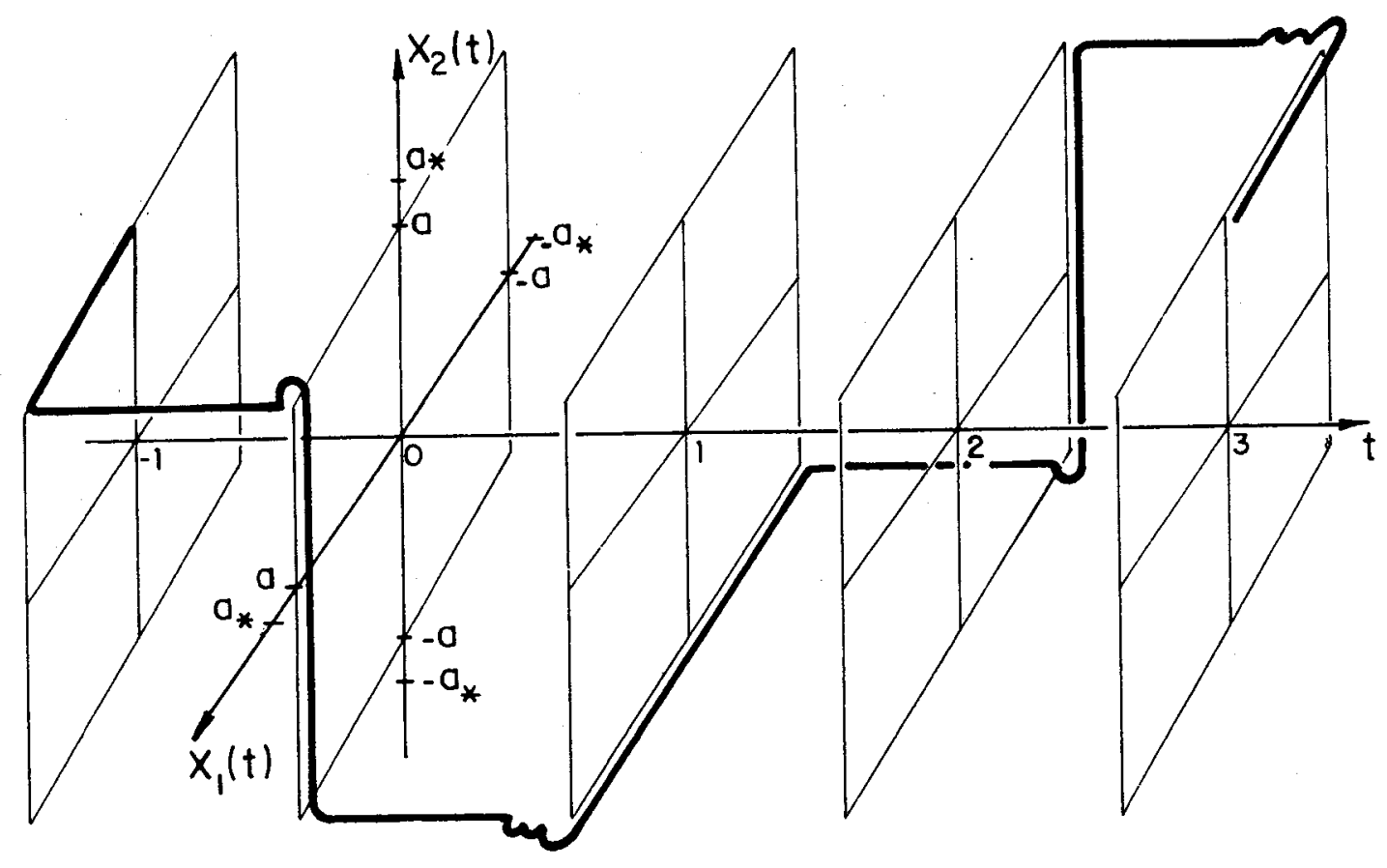

Figura II

Além disso, foram feitas algumas simulações no computador. A seguir, Exemplo 1 e Exemplo 2, são duas simulações que anexamos a este trabalho. 


\section{Exemplo 1:}

Consideremos o seguinte sistema:

$$
\left\{\begin{array}{l}
\varepsilon \dot{x}_{1}(t)=-x_{1}(t)+2 \operatorname{arctg} x_{2}(t-1) \\
\varepsilon \dot{x}_{2}(t)=-x_{2}(t)-2 \operatorname{arctg} x_{1}(t-1)
\end{array}\right.
$$

$\varepsilon>0$.

É fácil ver que a hipótese (H.1) está satisfeita. Além disso, $F\left(x_{1}, x_{2}\right)=$ $\left(2 \operatorname{arctg} x_{2},-2 \operatorname{arctg} x_{1}\right)$ é limitada e portanto, vale (H.2).

A hipótese (H.3) também está satisfeita.

Temos que $f=-f_{2}=f_{1}$ é monótona, onde $f_{1}$ e $f_{2}$ são as coordenadas da. função $F$. A função $f$ é de classe $C^{\infty}$ e ímpar, e $f([-A, A]) \subseteq[-A, A]$, onde $A \cong 2,3311$. Ainda, $|f f(x)|>|x|$ sempre que $x \in(-A, A)$ e $x \neq 0$.

Portanto, esse exemplo satisfaz as hipóteses do primeiro caso.

Desse modo, para particulares valores de $\varepsilon$, determinamos numericamente as $S^{*}$-soluções $x(t)=\left(x_{1}(t), x_{2}(t)\right)$ de $(\mathrm{I})_{\varepsilon}$. As próximas figuras, obtidas em computador, são dessas $S^{*}$-soluções.

Podemos verificar que, quando $\varepsilon \rightarrow 0_{+}, x(t)=\left(x_{1}(t), x_{2}(t)\right)$ converge uniformemente em subconjuntos compactos de $\mathbb{R}-\mathbb{Z}$ para a função "onda quadrada" $o \bar{q}(t)=\left(o \bar{q}_{1}(t), o \bar{q}_{2}(t)\right)$, dada por:

$$
\begin{aligned}
& o \bar{q}_{1}(t)=\left\{\begin{aligned}
2,3311 \ldots, & 4 n-1<t<4 n+1 \\
-2,3311 \ldots, & 4 n+1<t<4 n+3,
\end{aligned}\right. \\
& o \bar{q}_{2}(t)=\left\{\begin{aligned}
2,3311 \ldots, & 4 n-1<t<4 n \\
-2,3311 \ldots, & 4 n<t<4 n+2 \\
2,3311 \ldots, & 4 n+2<t<4 n+3, \quad n \in \mathbb{Z}, t \in \mathbb{R} .
\end{aligned}\right.
\end{aligned}
$$




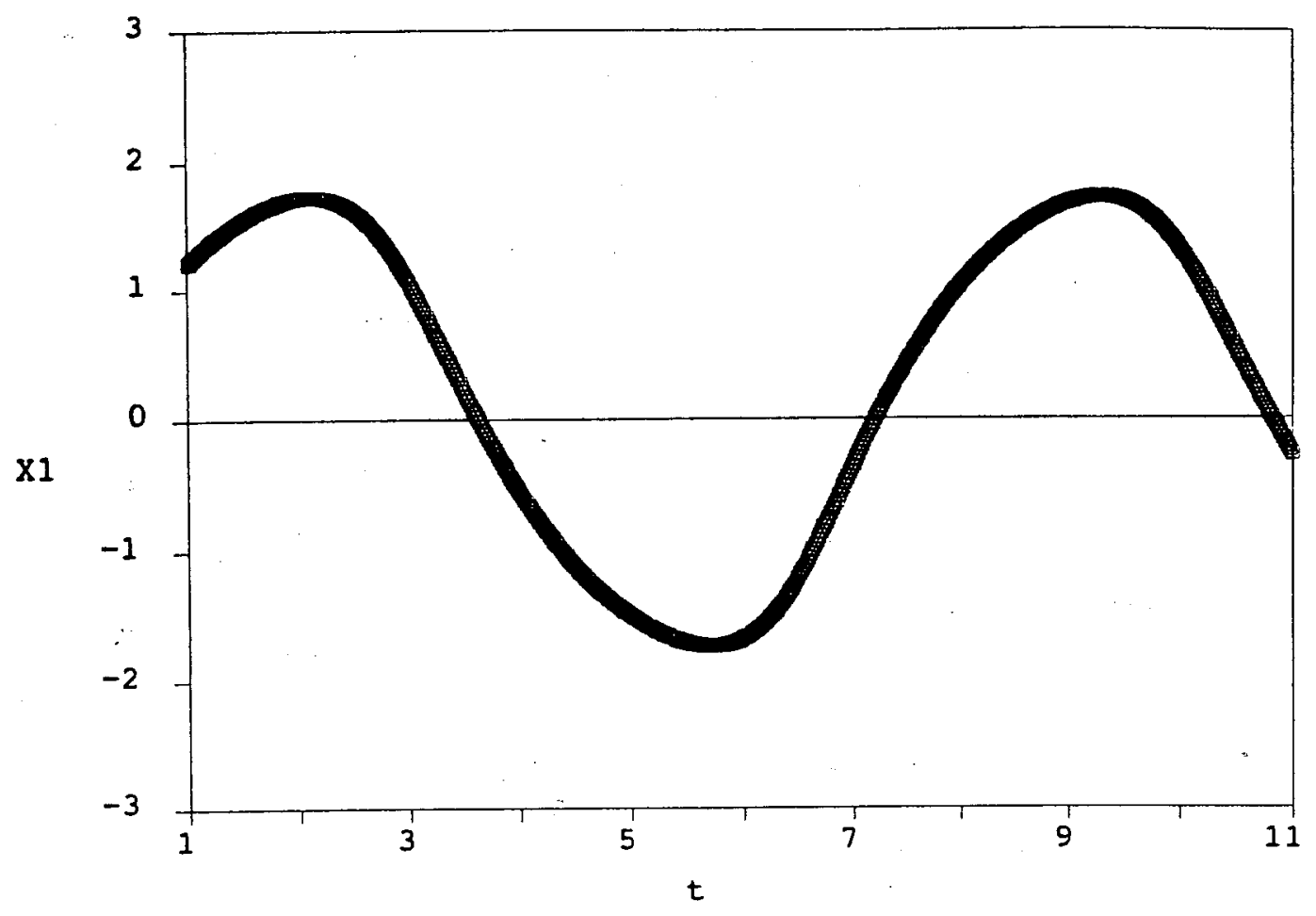

Figura 1.1: Gráfico da primeira coordenada de $x(t)$, solução de $(I)_{\varepsilon}, \varepsilon=1$.

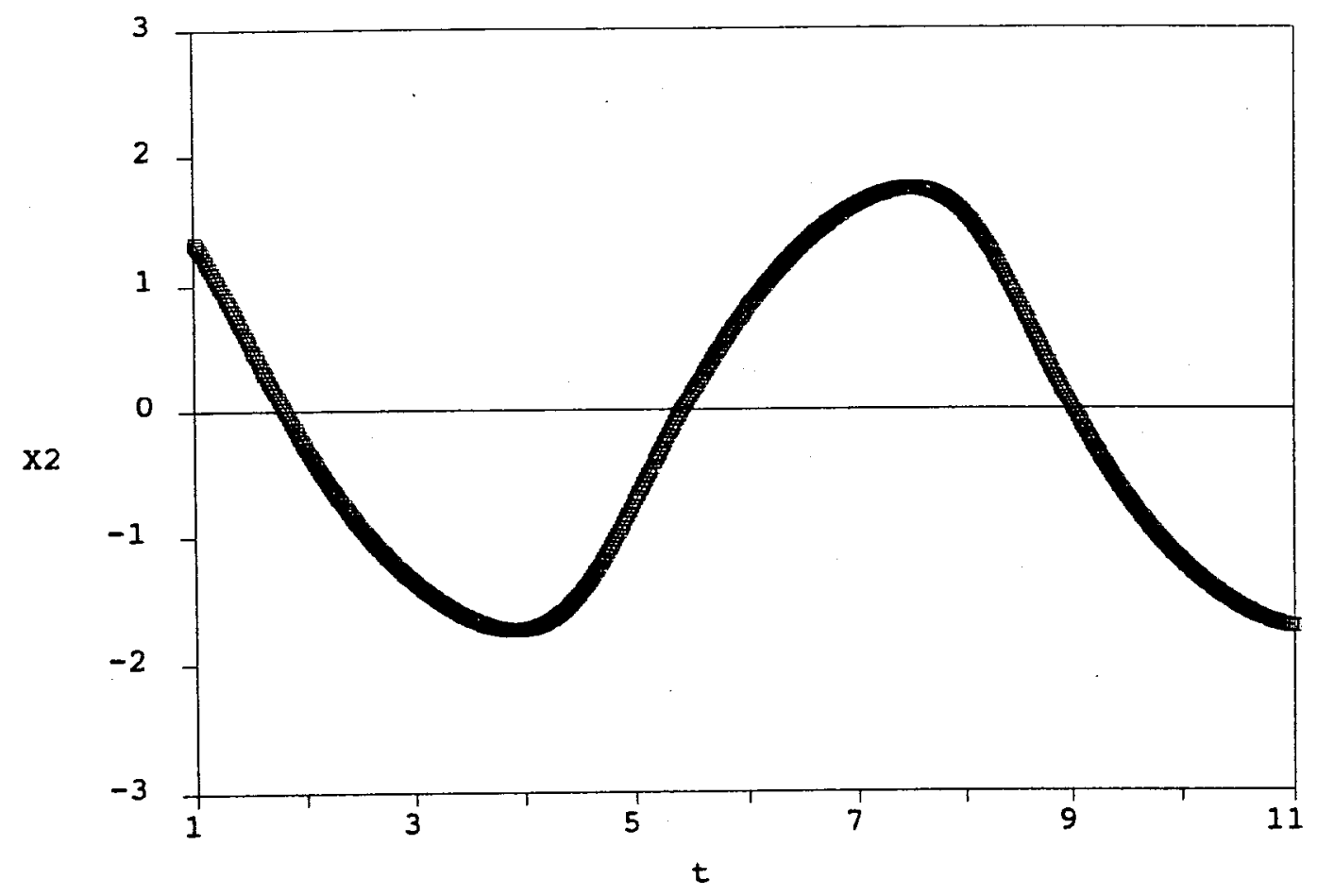

Figura 1.2: Gráfico da segunda coordenada de $x(t)$, solução de $(I)_{\varepsilon}, \varepsilon=1$. 


$$
\frac{v}{v}
$$




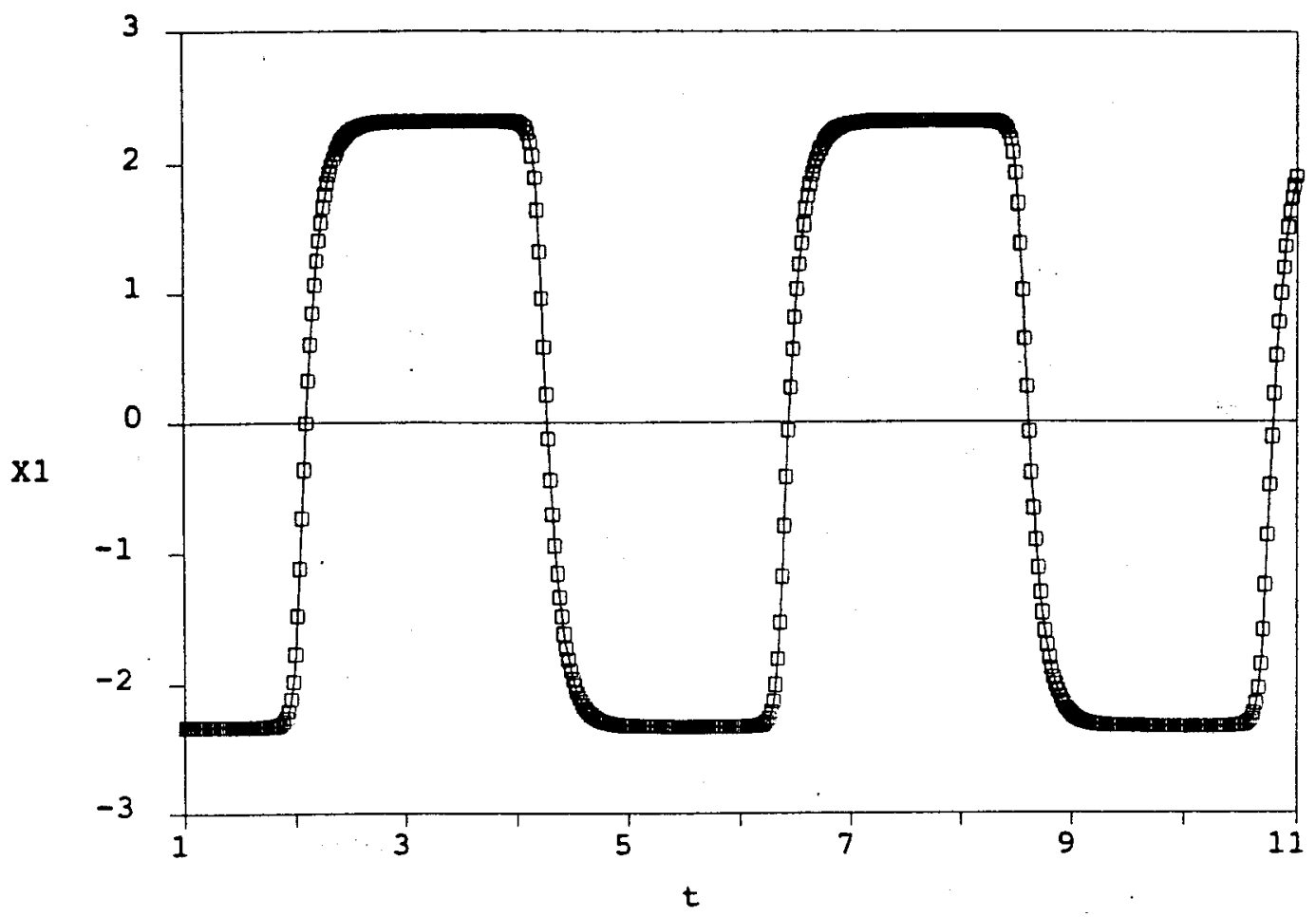

Figura 1.5: Gráfico da primeira coordenada de $x(t)$, solução de $(I)_{\varepsilon}, \varepsilon=0,1$.

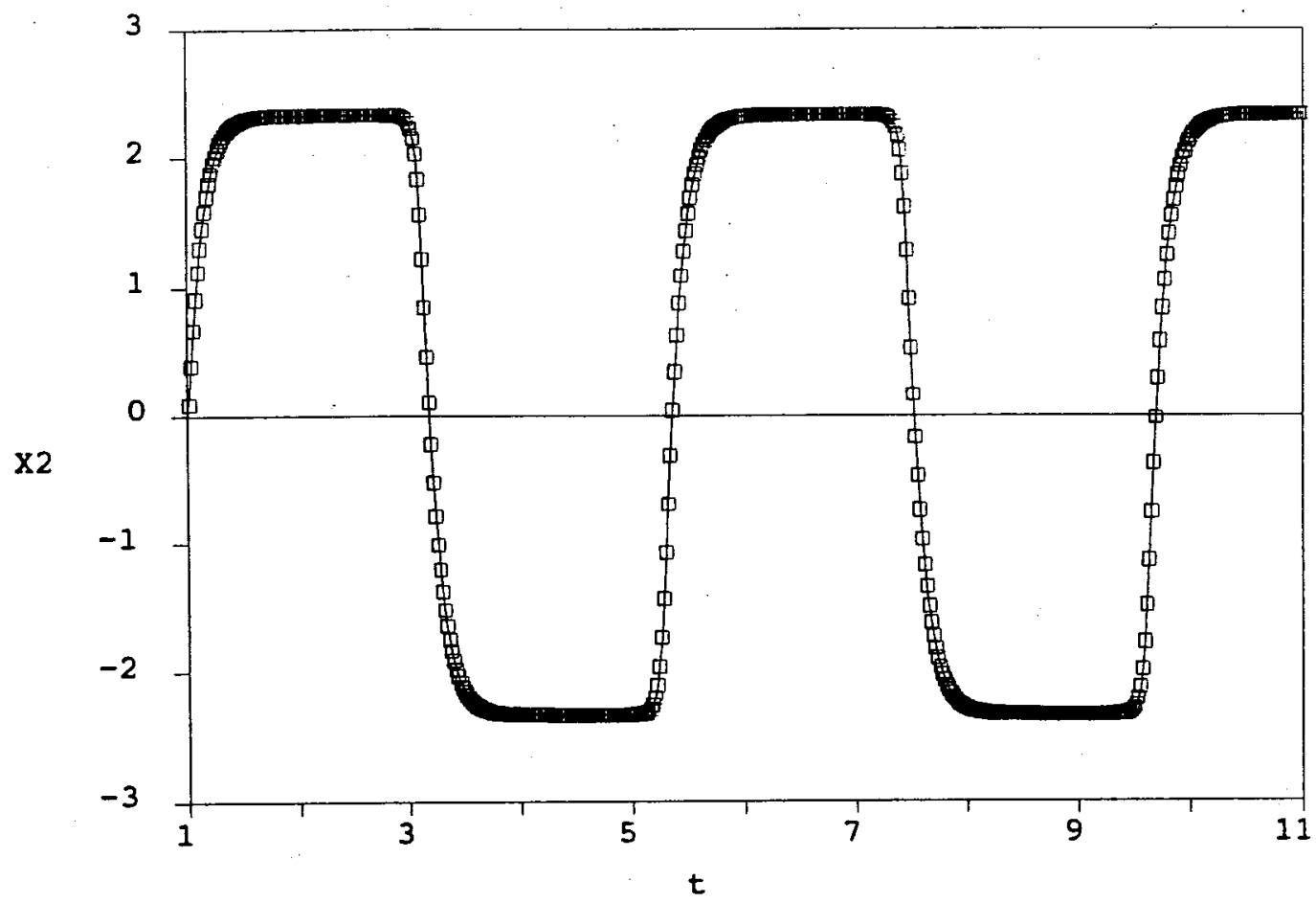

Figura 1.6: Gráfico da segunda coordenada de $x(t)$, solução de $(I)_{\varepsilon}, \varepsilon=0,1$. 


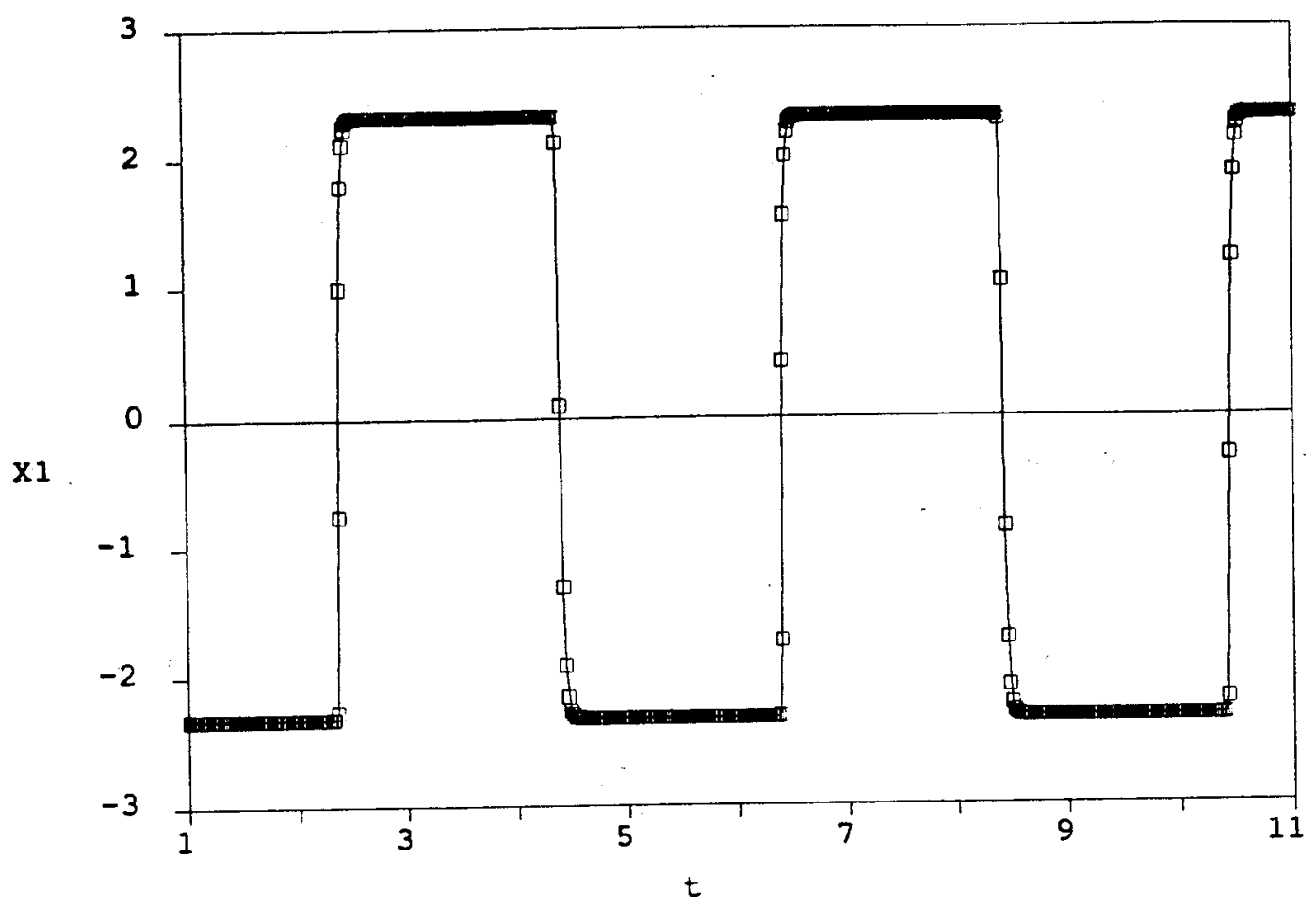

Figura 1.7: Gráfico da primeira coordenada de $x(t)$, solução de $(I)_{\varepsilon}, \varepsilon=0,01$.

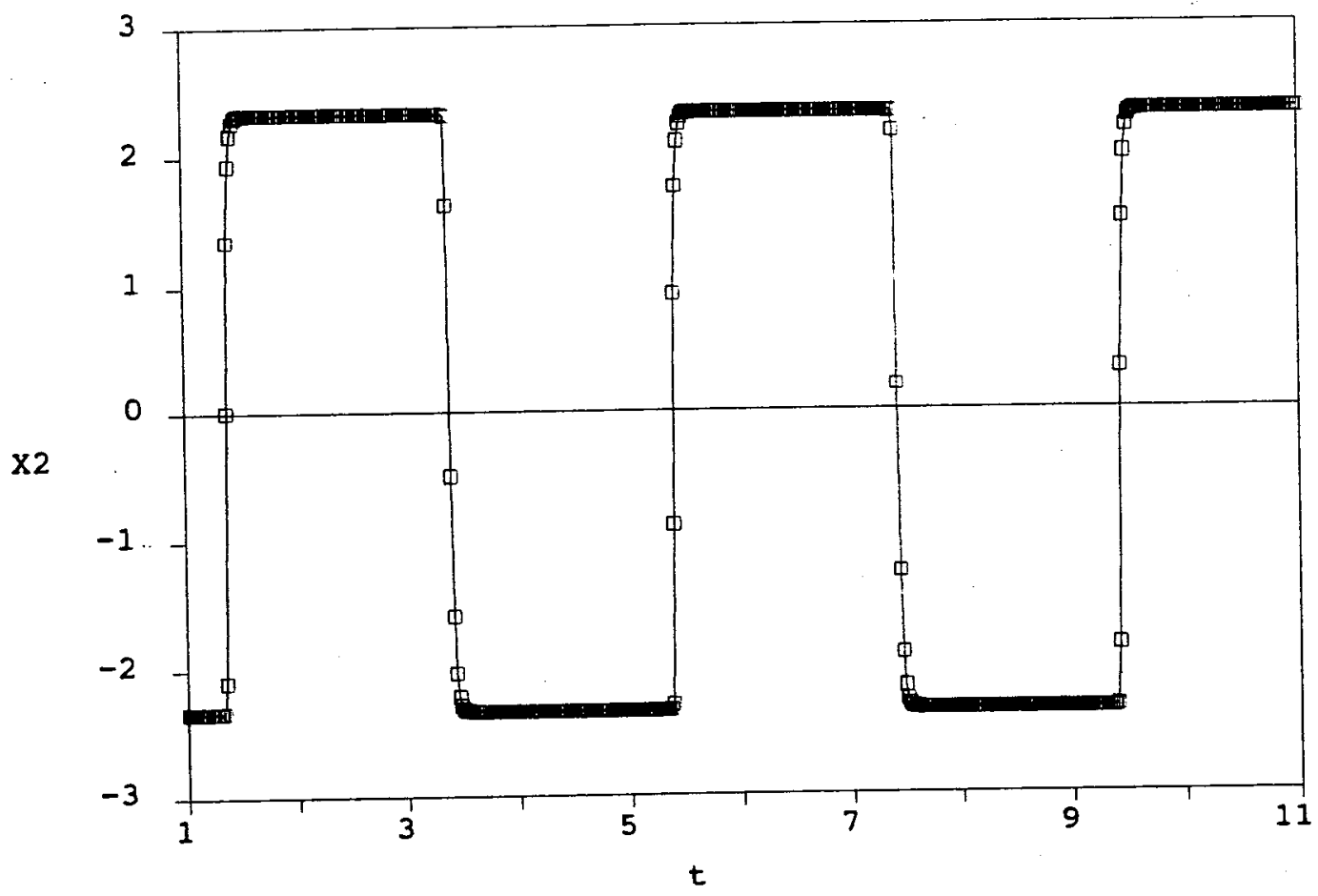

Figura 1.8: Gráfico da segunda coordenada de $x(t)$, solução de $(I)_{\varepsilon}, \varepsilon=0,01$. 


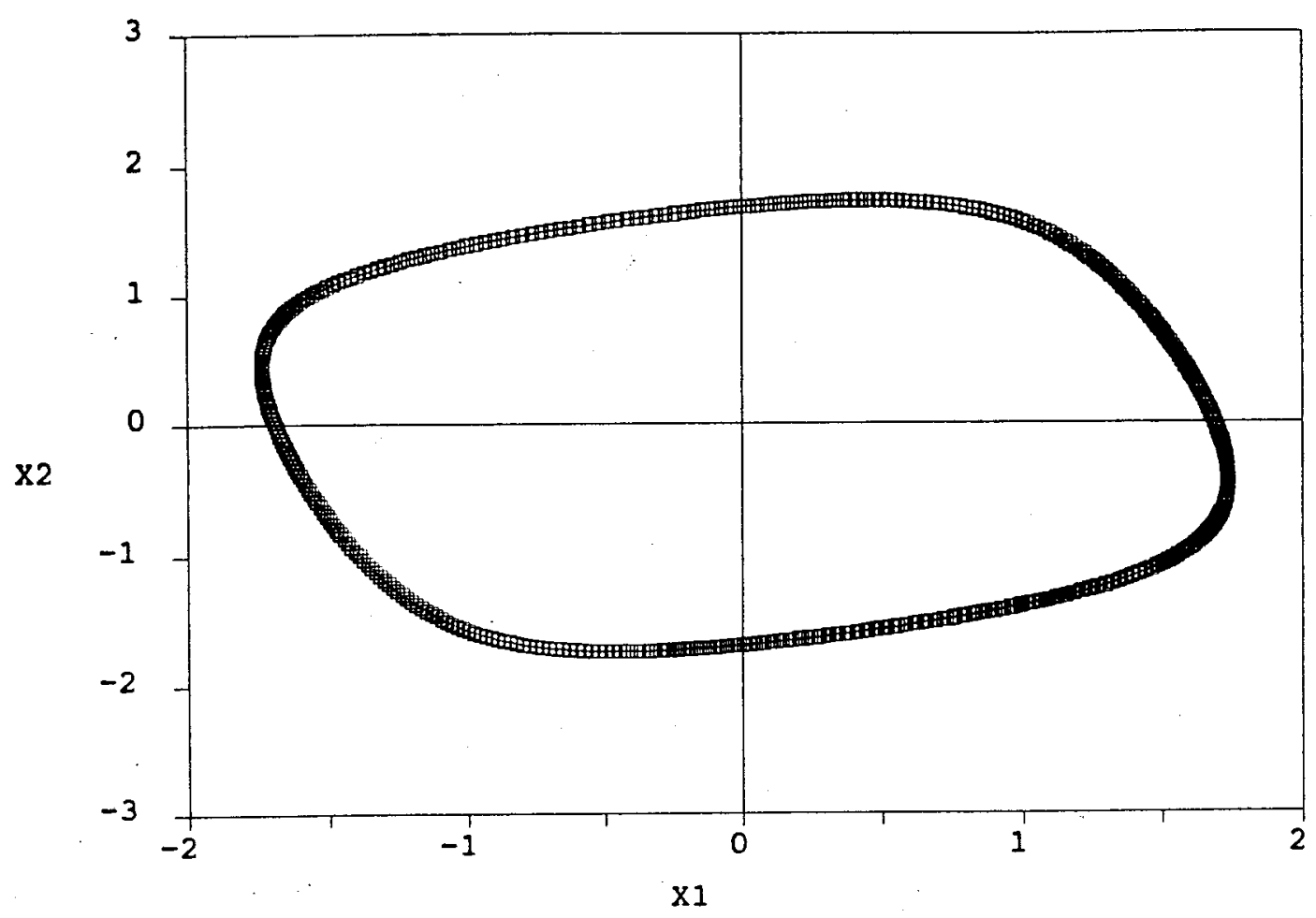

Figura 1.9: Gráfico da órbita de $x(t)$, solução de $(I)_{\varepsilon}, \varepsilon=1$.

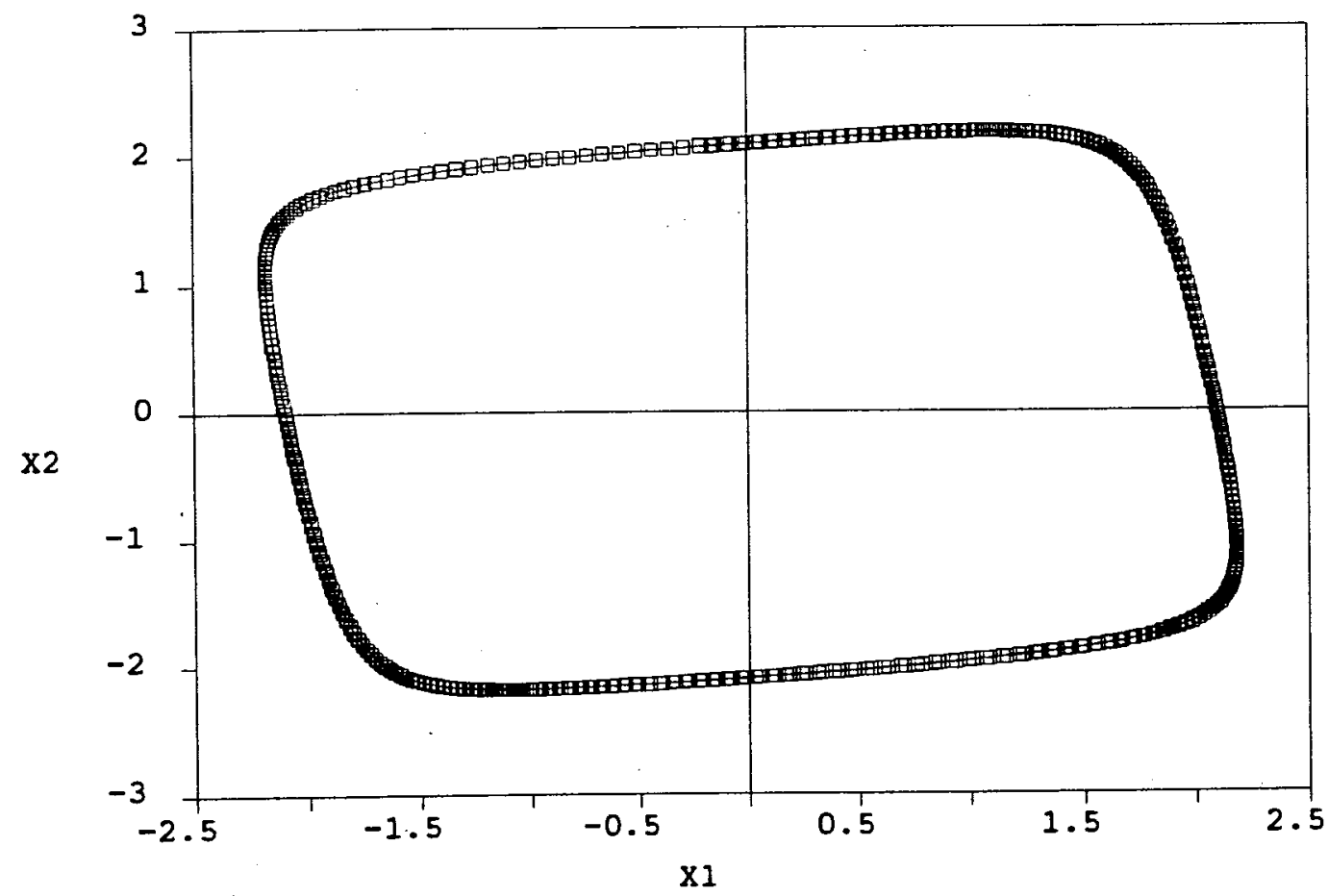

Figura 1.10: Gráfico da órbita de $x(t)$, soluçâo de $(I)_{\varepsilon}, \varepsilon=0,5$. 


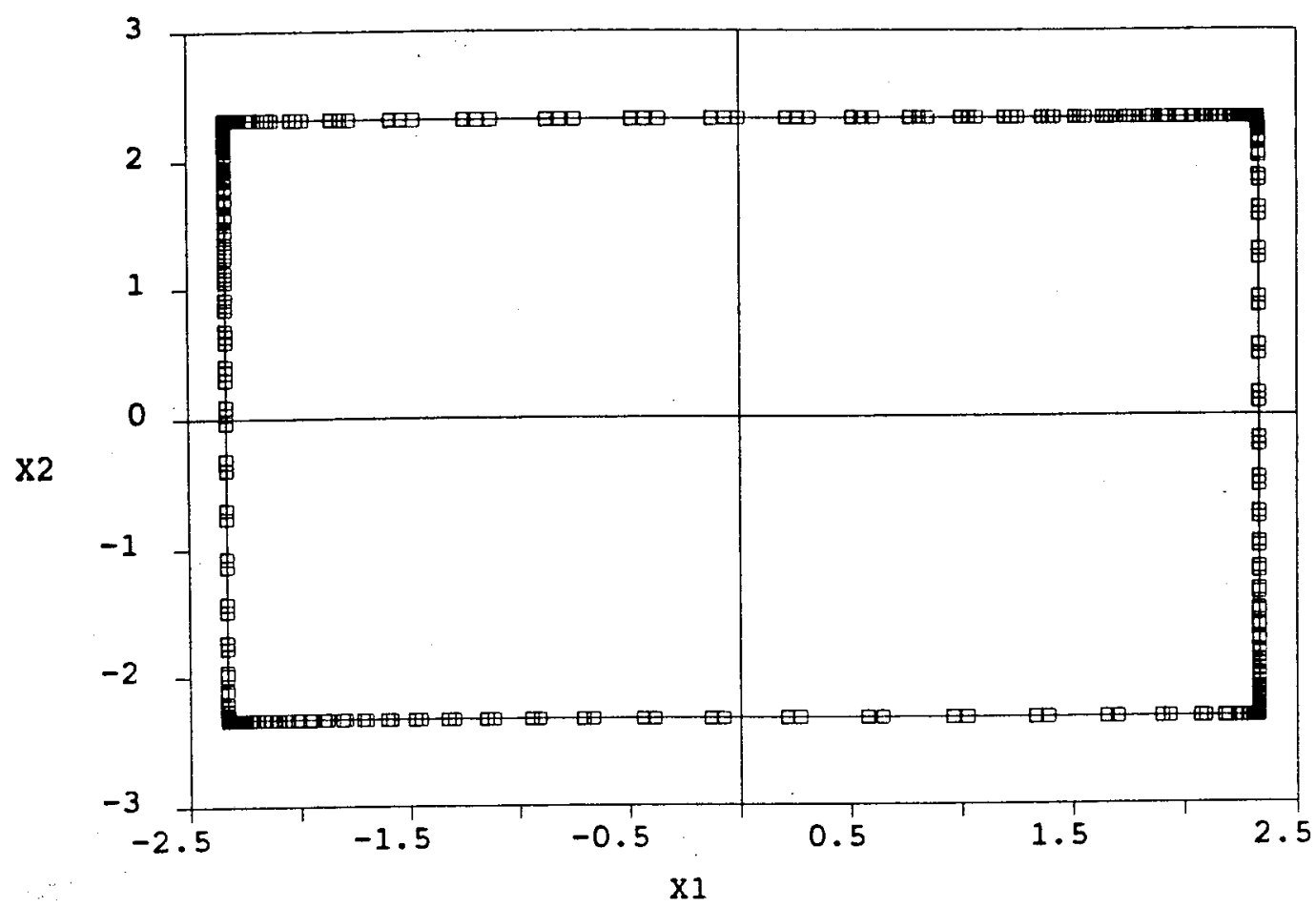

Figura 1.11: Gráfico da órbita de $x(t)$, solução de $(I)_{\varepsilon}, \varepsilon=0,1$.

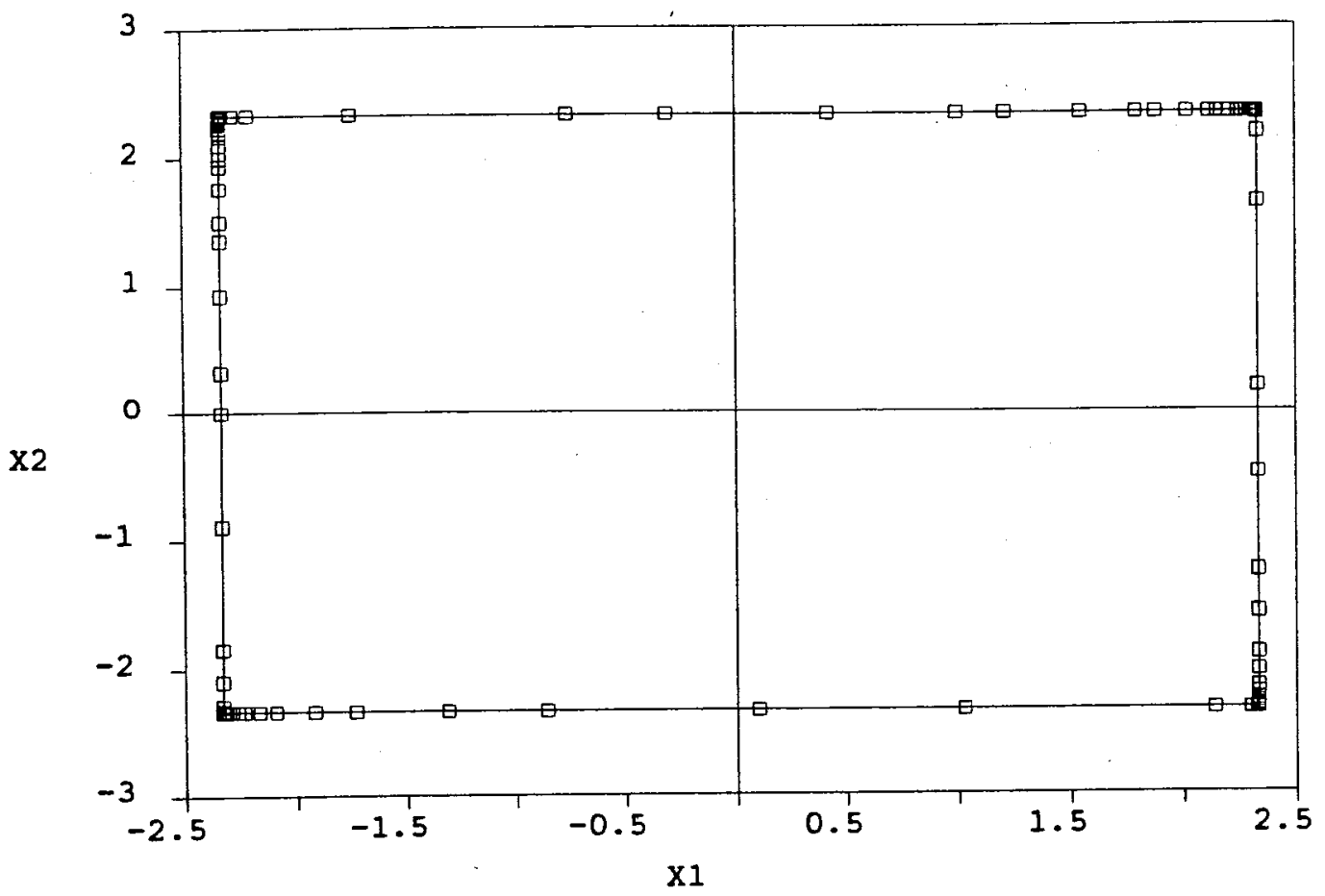

Figura 1.12: Gráfico da órbita de $x(t)$, solução de $(I)_{\varepsilon}, \varepsilon=0,01$. 


\section{Exemplo 2:}

Consideremos o seguinte sistema:

$$
\begin{aligned}
& \left\{\begin{array}{l}
\varepsilon \dot{x}_{1}(t)=-x_{1}(t)+\frac{6}{\pi} \cdot\left(\frac{1+x_{1}^{2}(t-1) \cdot x_{2}^{2}(t-1)}{1+2 \cdot x_{1}^{2}(t-1) \cdot x_{2}^{2}(t-1)}\right) \cdot \operatorname{arctg} x_{2}(t-1) \\
\varepsilon \dot{x}_{2}(t)=-x_{2}(t)-\frac{6}{\pi} \cdot\left(\frac{1+x_{1}^{2}(t-1) \cdot x_{2}^{2}(t-1)}{1+2 \cdot x_{1}^{2}(t-1) \cdot x_{2}^{2}(t-1)}\right) \cdot \operatorname{arctg} x_{1}(t-1),
\end{array}\right. \\
& \varepsilon>0 .
\end{aligned}
$$

Nesse exemplo as componentes de $F=\left(F_{1}, f_{2}\right)$ dependem efetivamente das duas variáveis $x_{1}$ e $x_{2}$.

É fácil verificar que :

$$
\begin{aligned}
& x_{2} \cdot F_{1}(x)>0, \quad x_{2} \neq 0 \\
& x_{1} \cdot F_{2}(x)<0, \quad x_{1} \neq 0, x=\left(x_{1}, x_{2}\right) \text { e } \delta=\sqrt{-\delta_{1} \cdot \delta_{2}}>1,
\end{aligned}
$$

onde $\delta_{1}=\frac{\partial F_{1}}{\partial x_{2}}(0), \delta_{2}=\frac{\partial F_{2}}{\partial x_{1}}(0)$.

Além disso,

$$
F\left(x_{1}, x_{2}\right)=\left(\frac{6}{\pi} \cdot\left(\frac{1+x_{1}^{2} \cdot x_{2}^{2}}{1+2 \cdot x_{1}^{2} \cdot x_{2}^{2}}\right) \cdot \operatorname{arctg} x_{2},-\frac{6}{\pi} \cdot\left(\frac{1+x_{1}^{2} \cdot x_{2}^{2}}{1+2 \cdot x_{1}^{2} \cdot x_{2}^{2}}\right) \cdot \operatorname{arctg} x_{1}\right)
$$

é limitada.

A hipótese (H.3) também está satisfeita.

Ainda, $\widetilde{F}=F_{1}=-F_{2}$ e $\tilde{F}\left(x_{1}, x_{2}\right)=-\widetilde{F}\left(-x_{1},-x_{2}\right)$.

O ponto $(1,1)$ é quatro periódico para a função $F$, isto é, $F^{4}(1,1)=(1,1)$.

Desse modo, para particulares valores de $\varepsilon$, determinamos numericamente as $S^{*}$-soluções $x(t)=\left(x_{1}(t), x_{2}(t)\right)$ de (II) $\varepsilon$. As próximas figuras, obtidas em computador, são dessas $S^{*}$-soluções.

Esse exemplo indica que, resultados podem ser investigados, em situações mais gerais da que foi estudada aqui. 


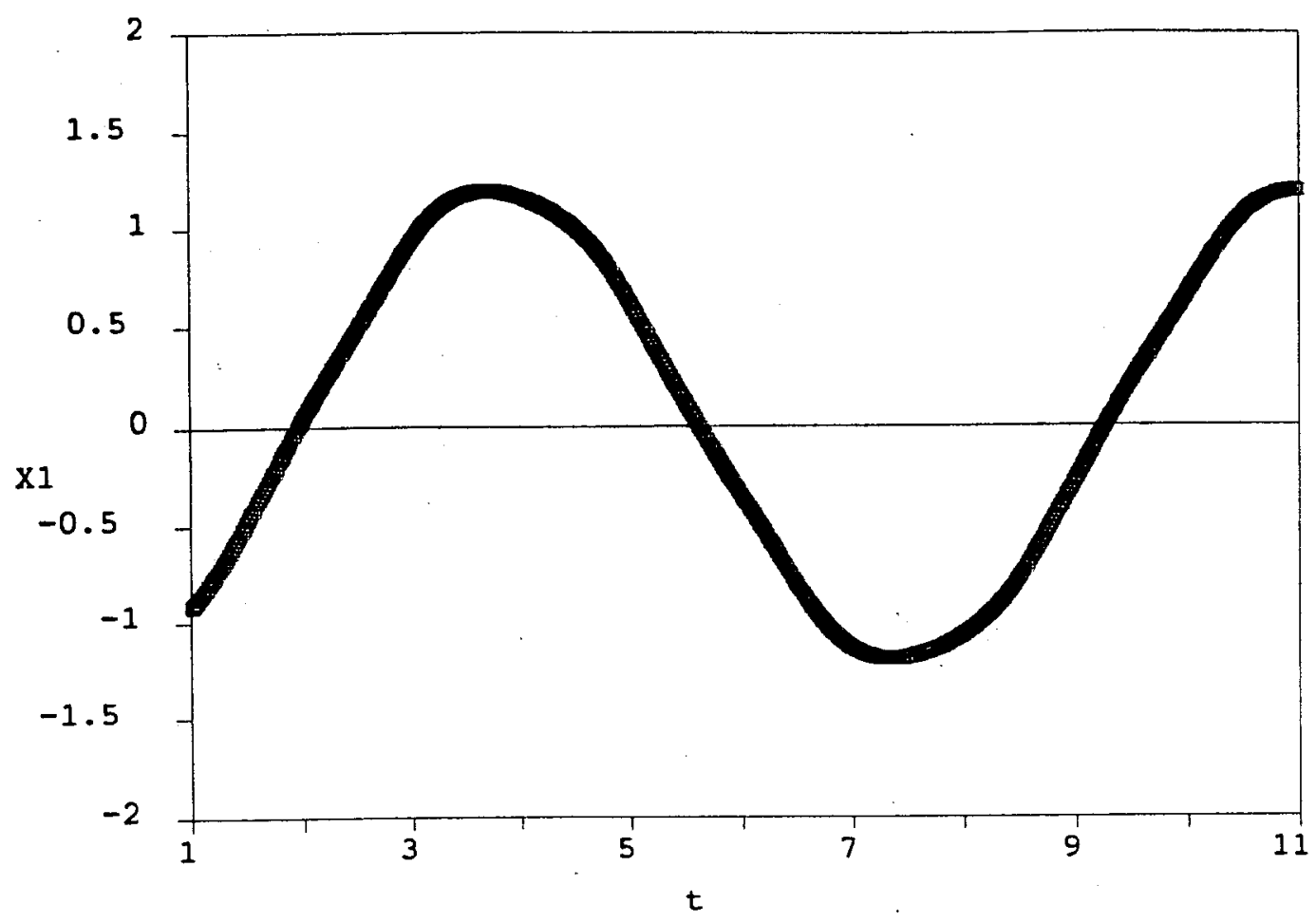

Figura 2.1: Gráfico da primeira coordenada de $x(t)$, solução de $(I I)_{\varepsilon}, \varepsilon=1$.

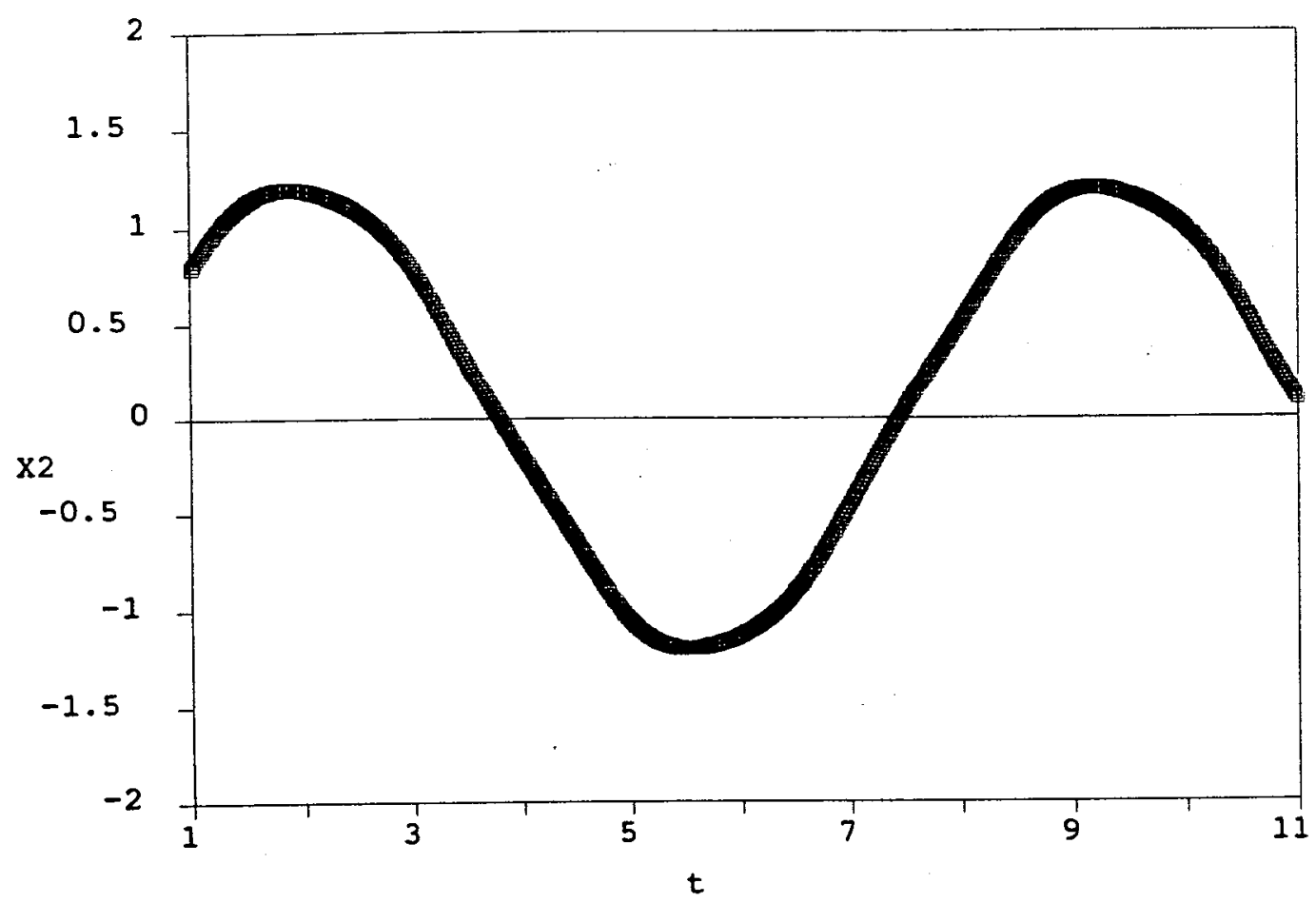

Figura 2.2: Gráfico da segunda coordenada de $x(t)$, solução de $(I I)_{\varepsilon}, \varepsilon=1$. 


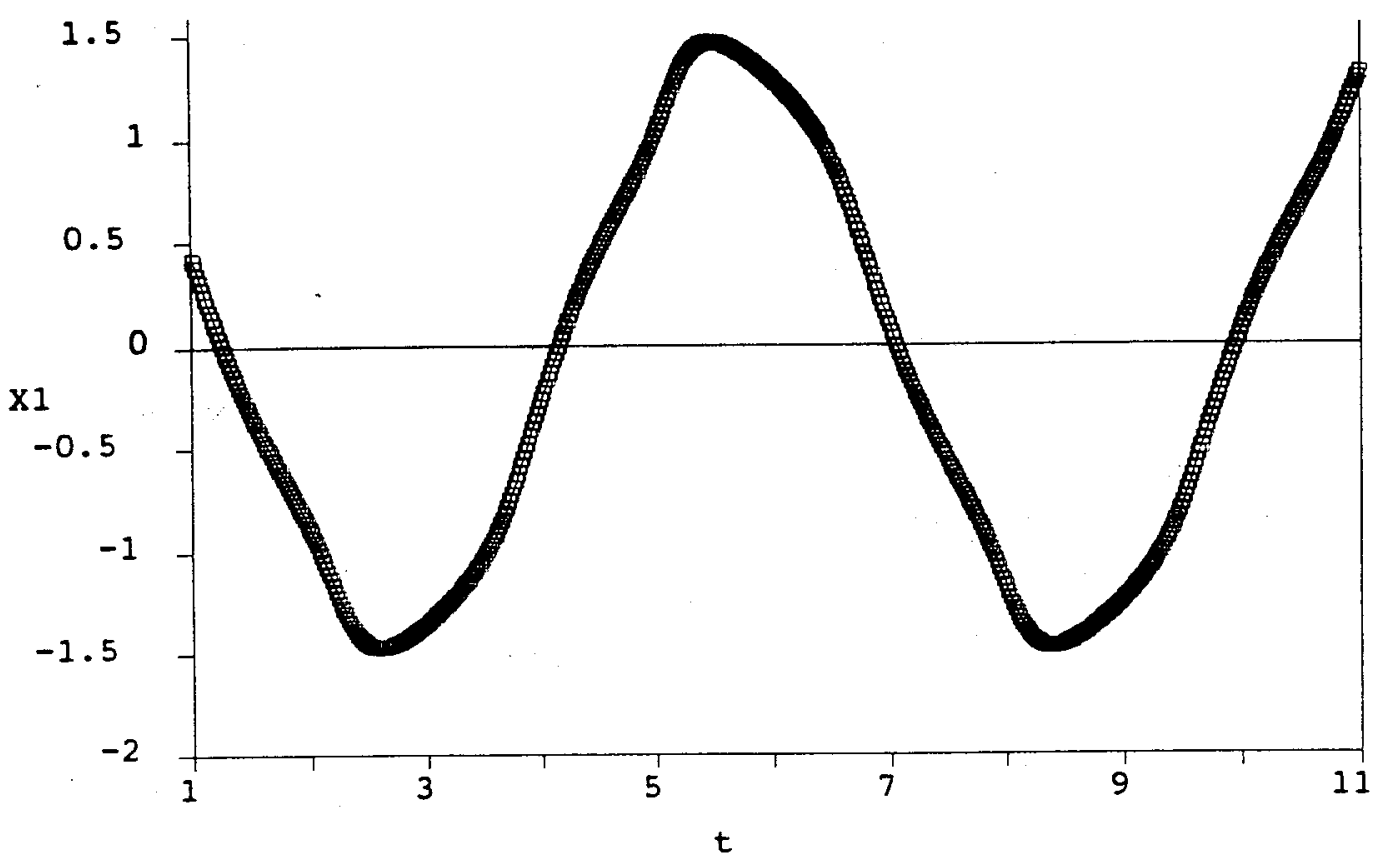

Figura 2.3: Gráfico da primeira coordenada de $x(t)$, solução de $(I I)_{\varepsilon}, \varepsilon=0,5$.

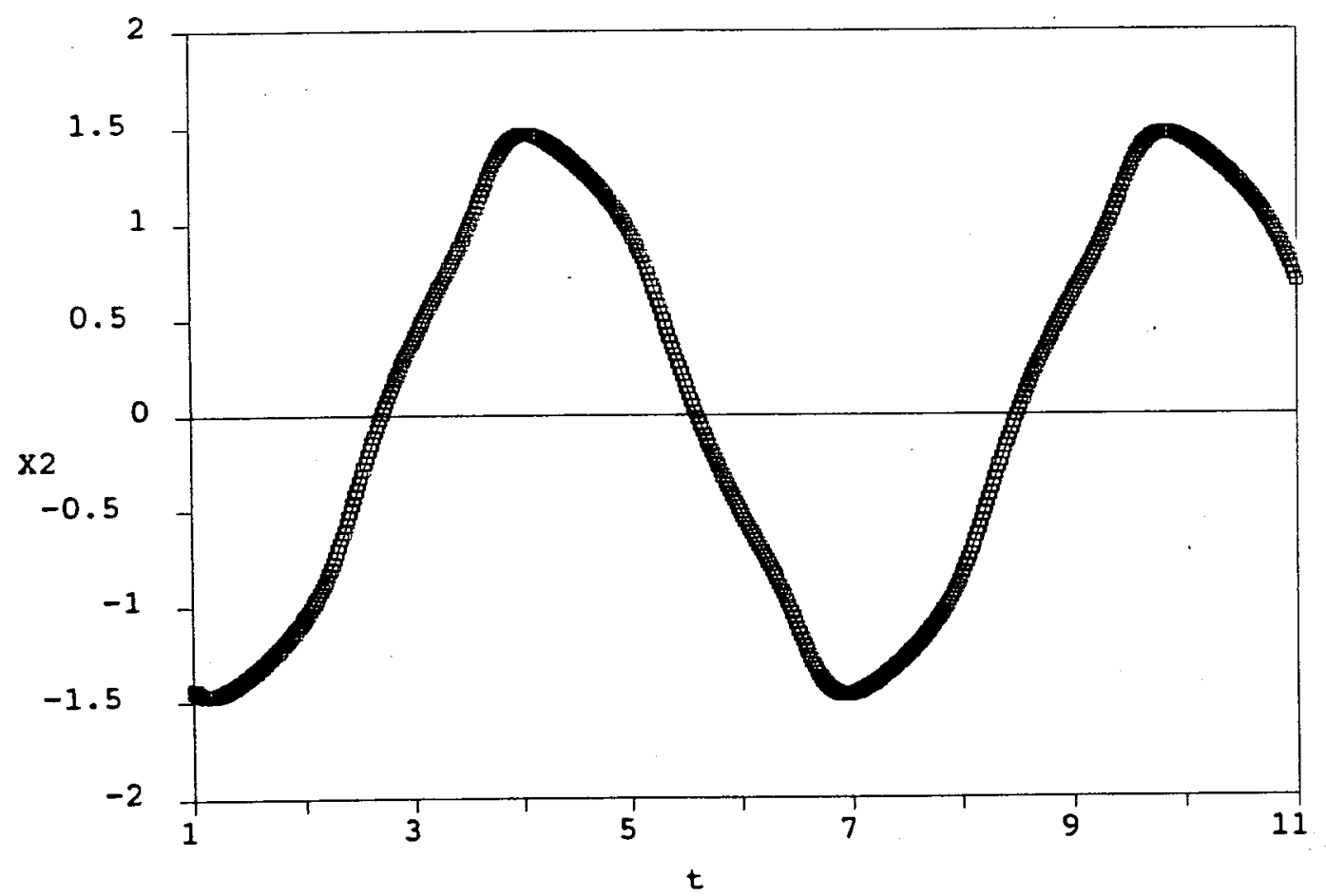

Figura 2.4: Gráfico da segunda coordenada de $x(t)$, solução de $(I I) \varepsilon, \varepsilon=0,5$. 


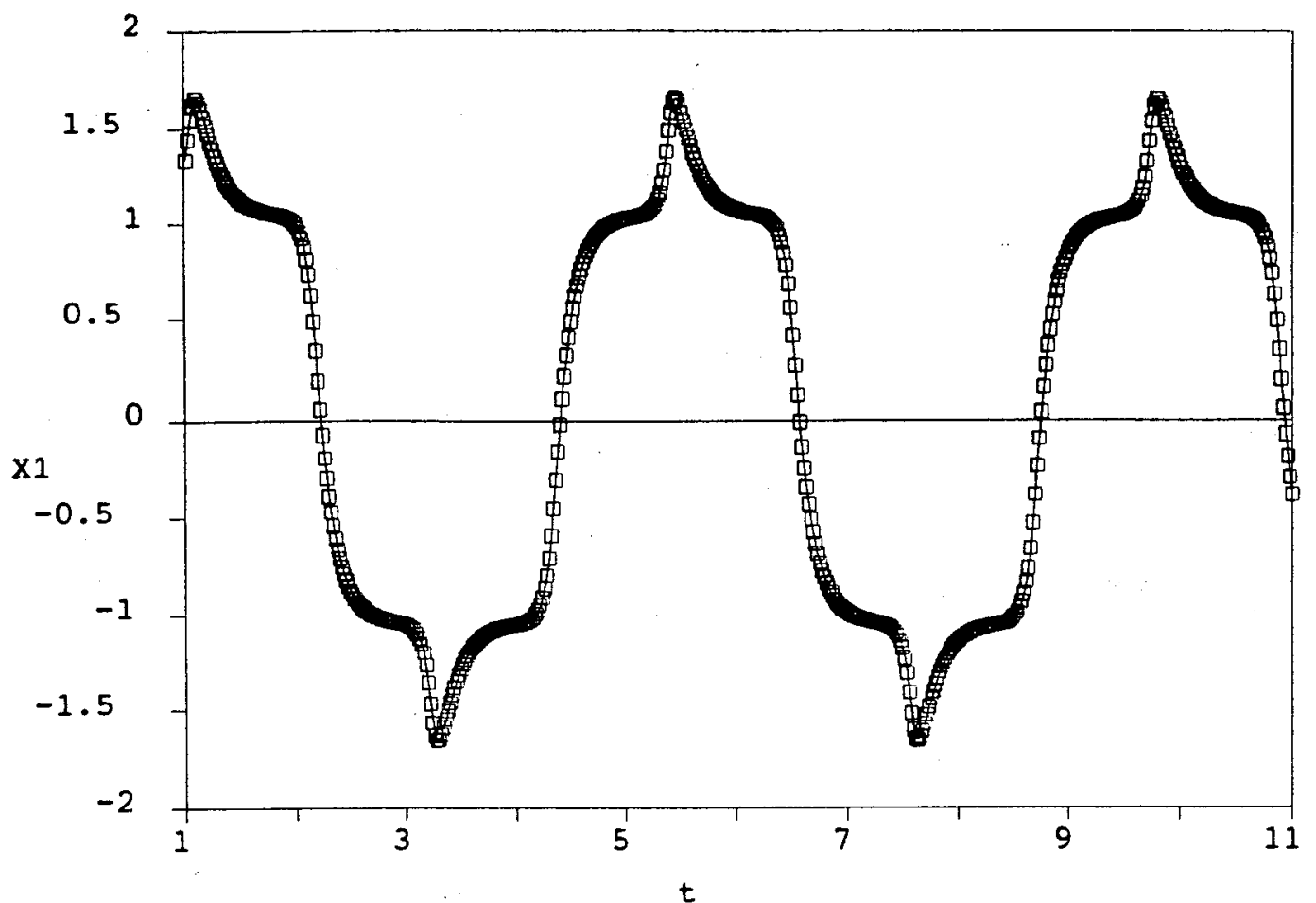

Figura 2.5: Gráfico da primeira coordenada de $x(t)$, solução de $(I I)_{\varepsilon}, \varepsilon=0,1$.

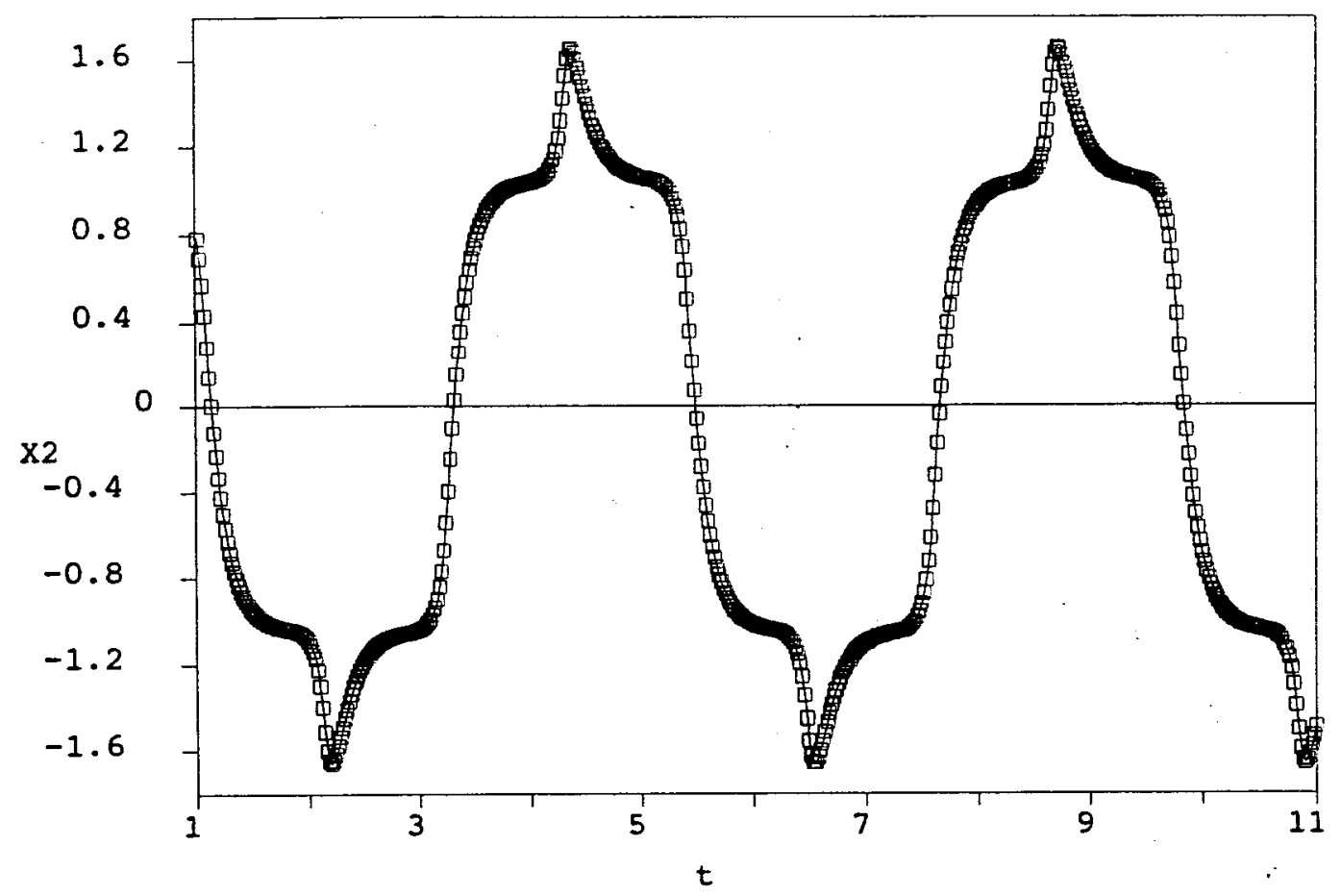

Figura 2.6: Gráfico da segunda coordenada de $x(t)$, solução de $(I I)_{\varepsilon}, \varepsilon=0,1$. 


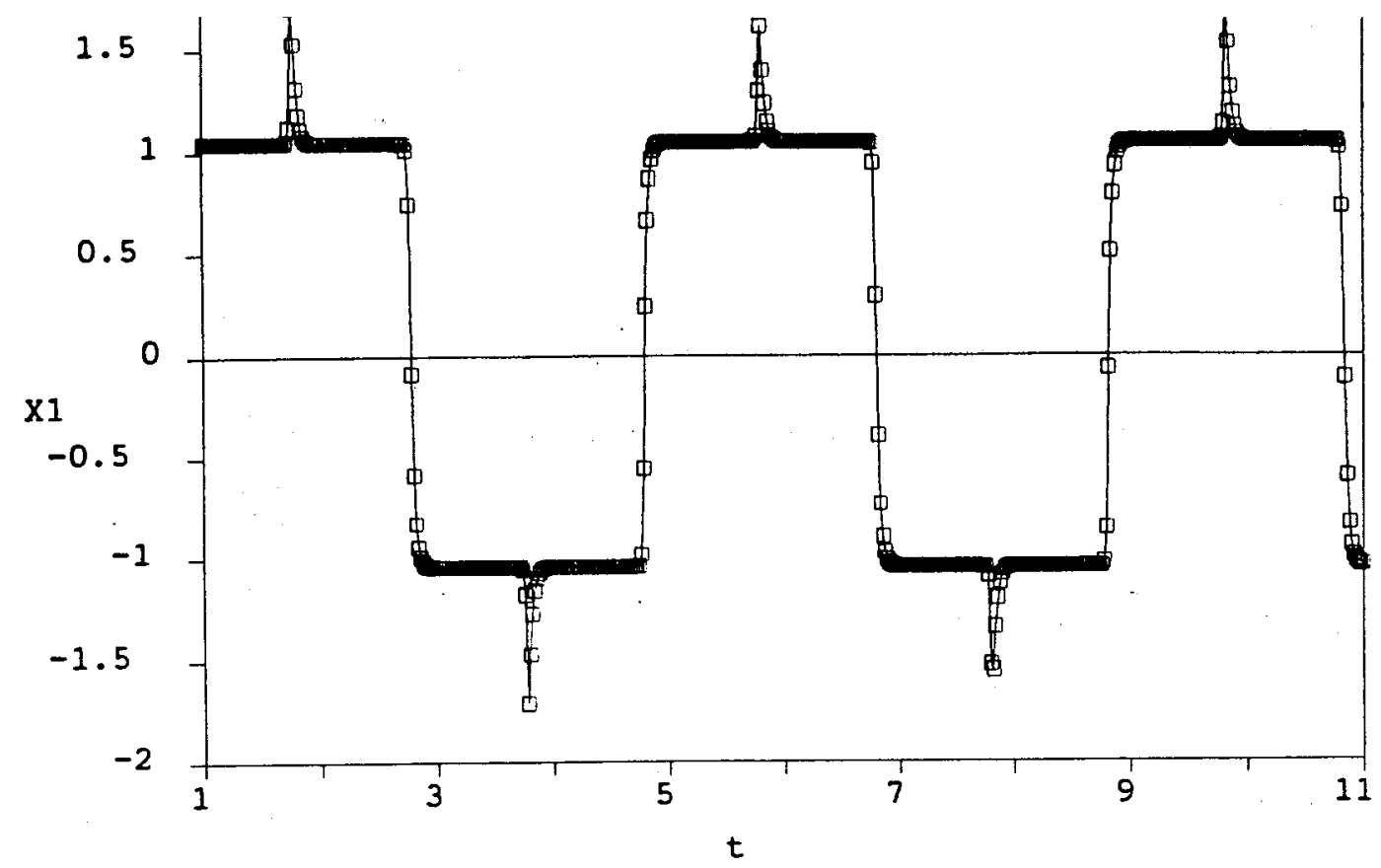

Figura 2.7: Gráfico da primeira coordenada de $x(t)$, solução de $(I I)_{\varepsilon}, \varepsilon=0,01$.

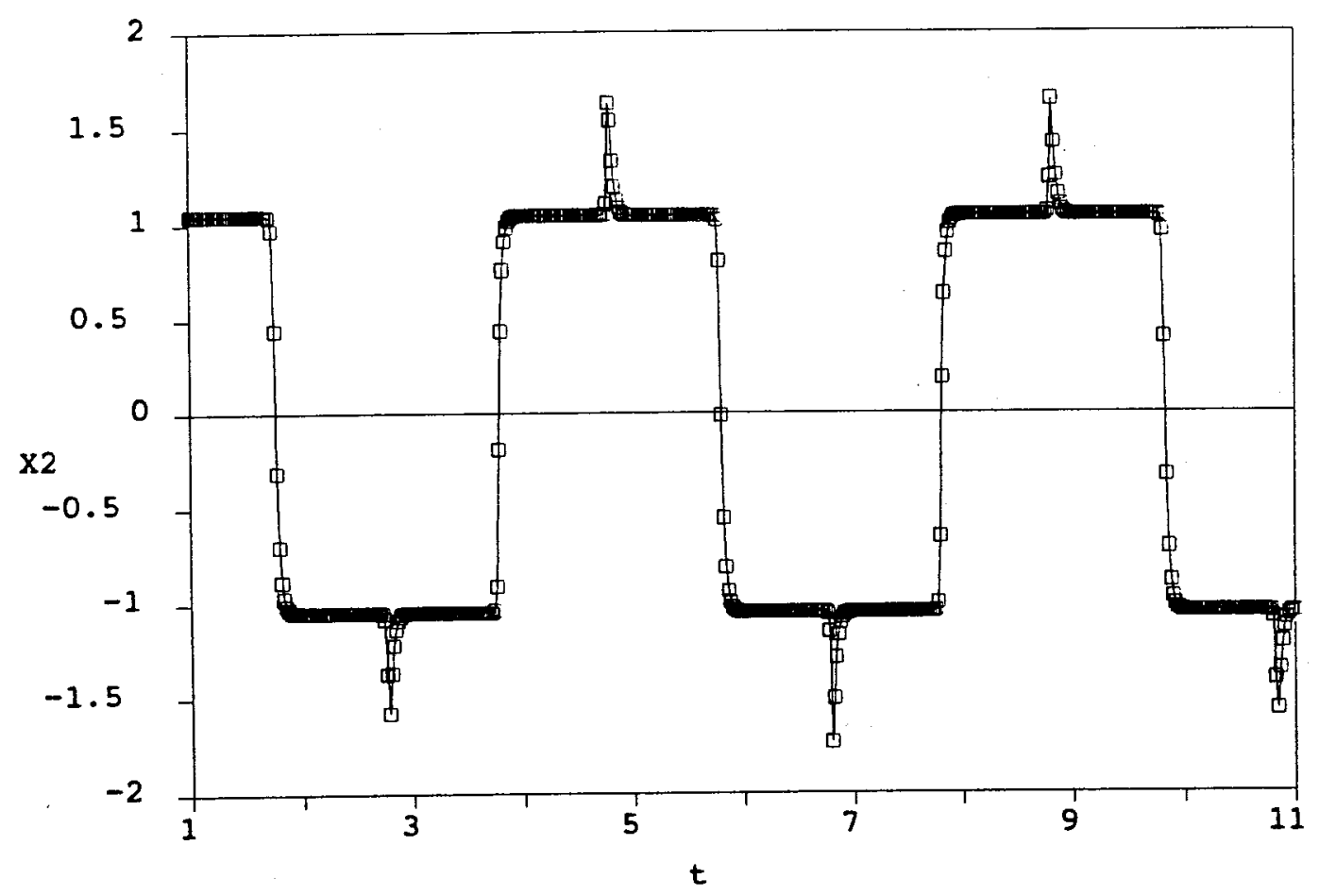

Figura 2.8: Gráfico da segunda coordenada de $x(t)$, solução de $(I I)_{\varepsilon}, \varepsilon=0,01$. 


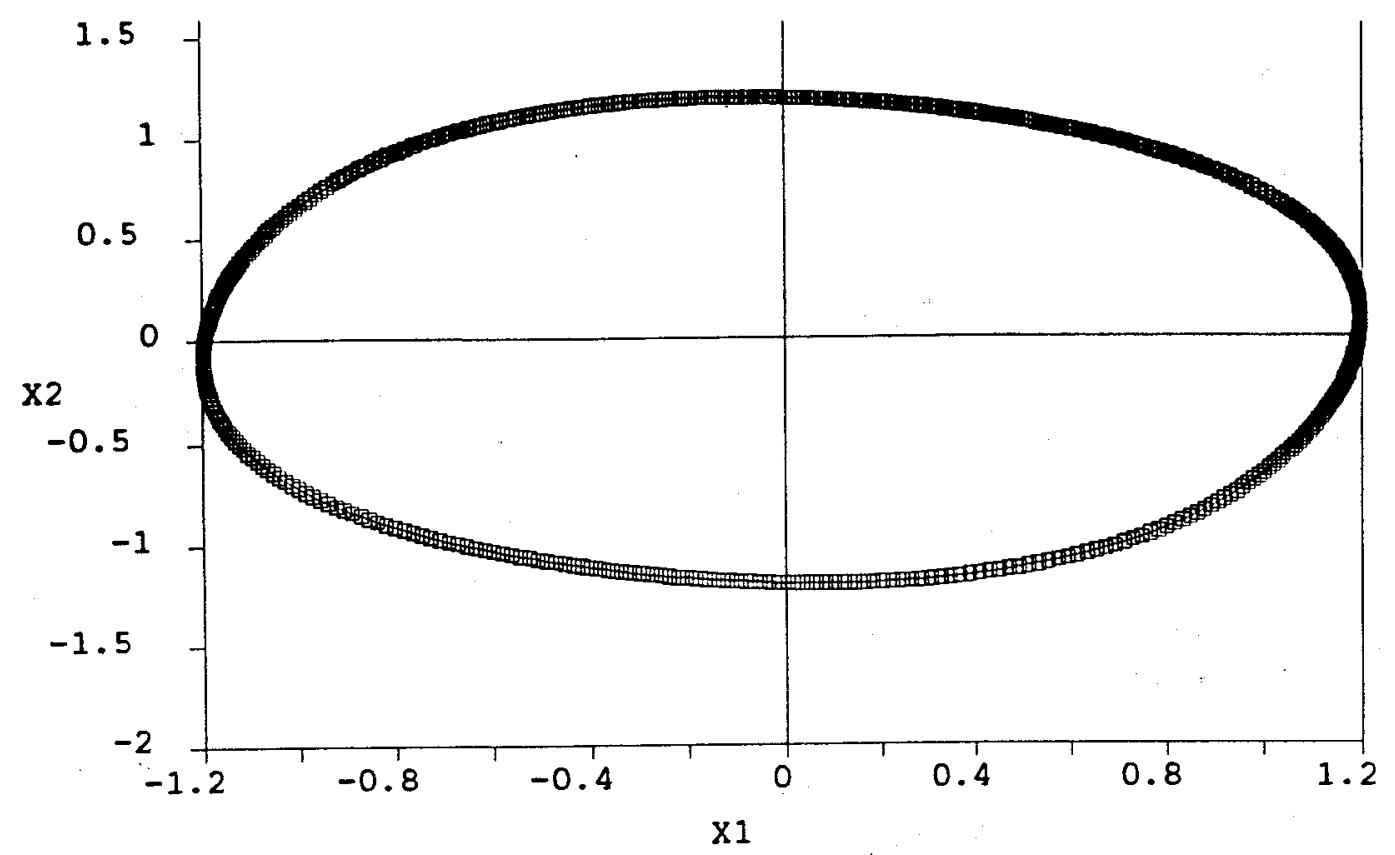

Figura 2.9: Gráfico da órbita de $x(t)$, solução de $(I I)_{\varepsilon}, \varepsilon=1$.

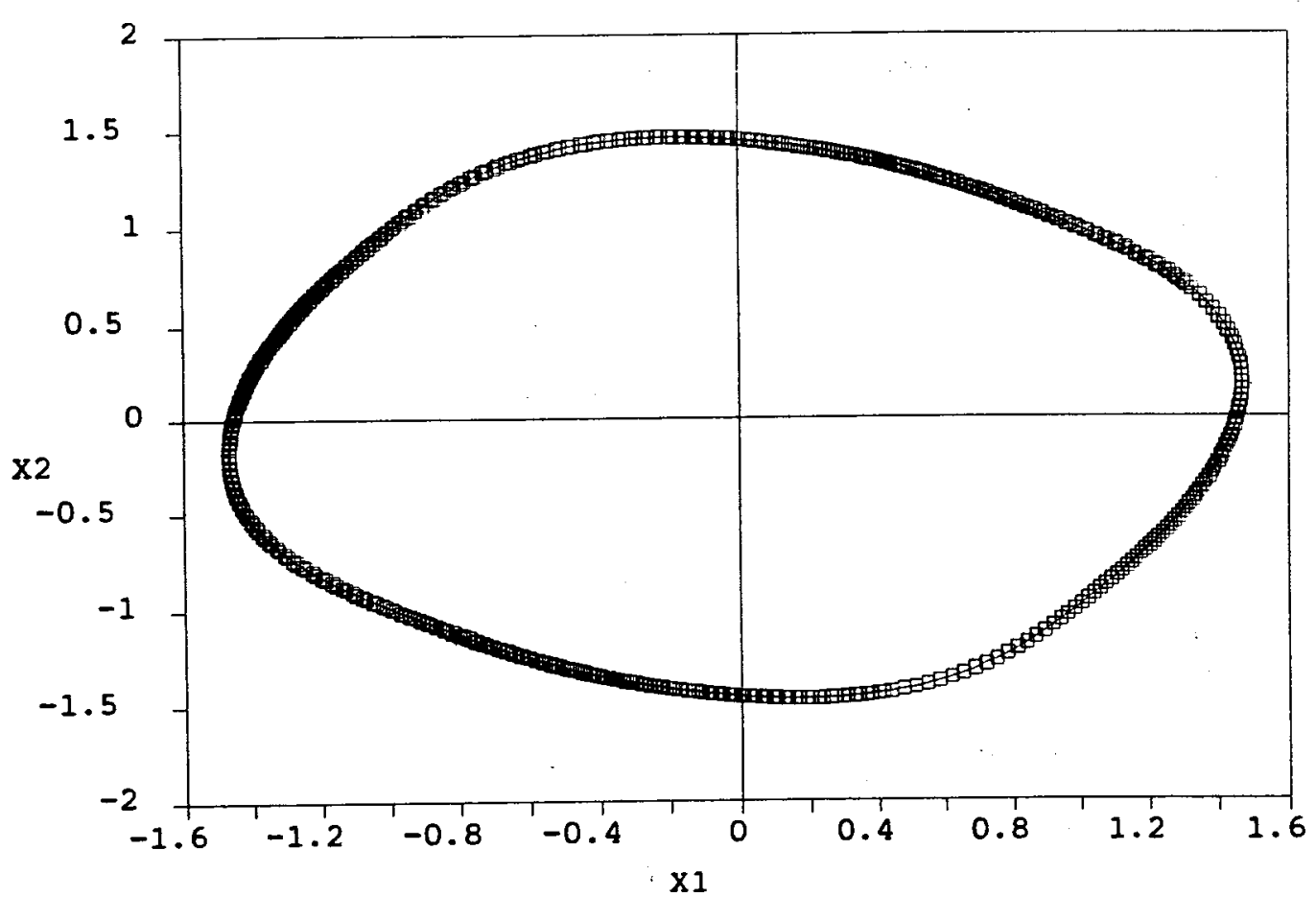

Figura 2.10: Gráfico da órbita de $x(t)$, solução de $(I I)_{\varepsilon}, \varepsilon=0,5$. 


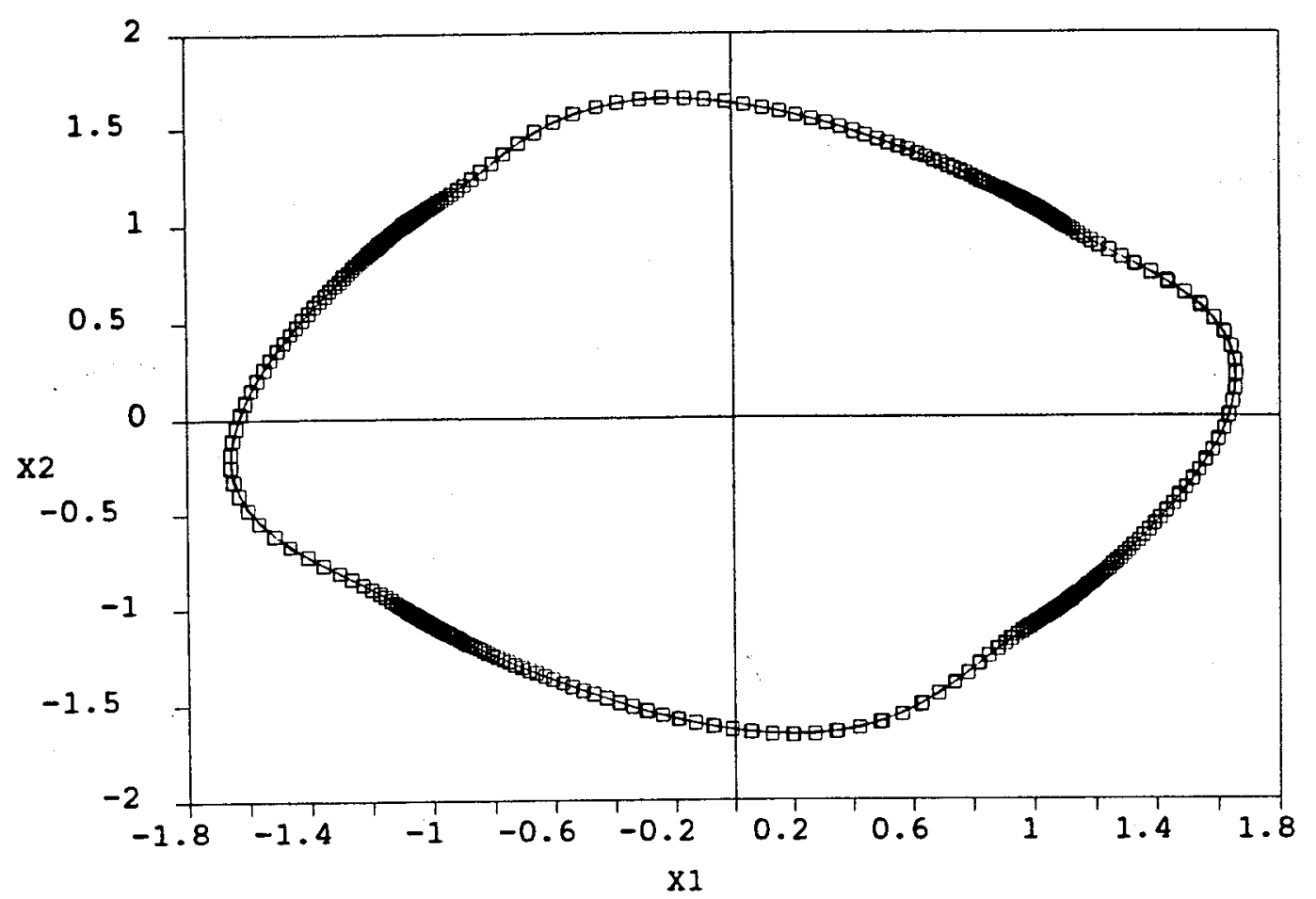

Figura 2.11: Gráfico da órbita de $x(t)$, solução de $(I I)_{\varepsilon}, \varepsilon=0,1$.

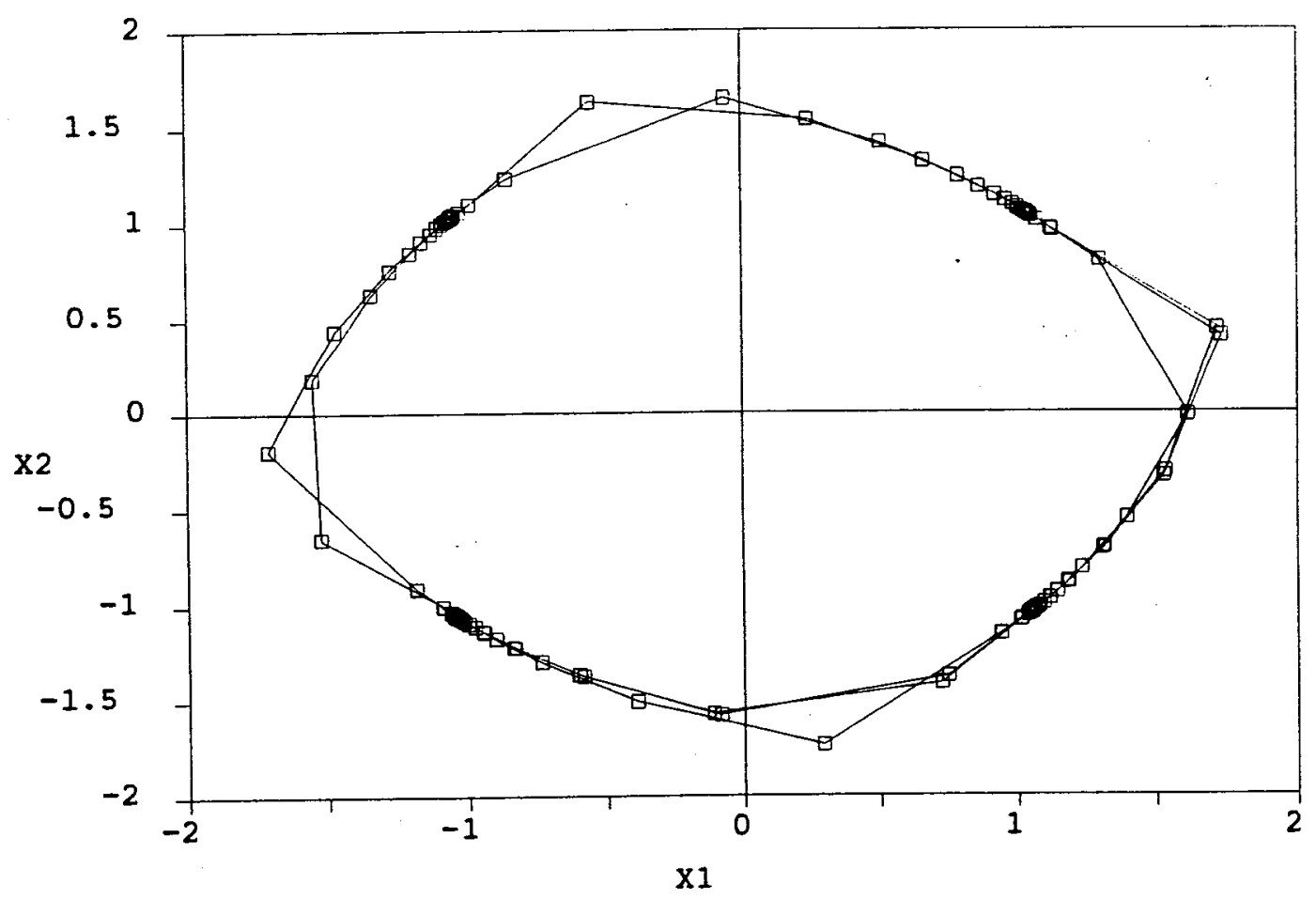

Figura 2.12: Gráfico da órbita de $x(t)$, solução de $(I I)_{\varepsilon}, \varepsilon=0,01$. 


\section{Bibliografia}

[1] J. Mallet-Paret; R. Nussbaum, Global continuation and asymptotic behavior for periodic solutions of a differential-delay equation, Ann. Mat. Pura IV, vol CXLV (1986), 33-128.

[2] M.Z. BAPTistinı, Soluções de uma equação diferencial no plano com retardamento e continuação global, Tese de doutorado (Brasil), Instituto de Ciências Matemáticas de São Carlos da Universidade de São Paulo, 1990.

[3] P.Z. TÁboas, Periodic solutions of a planar delay equation, Proc. Royal Soc. Edinburgh, 116 A (1990), 85-101.

[4] J.K. HALE, Theory of functional differential equations. 1ạ edição. New York, Springer-Verlag, 1977, $365 \mathrm{p}$.

[5] R.D. Nussbaum, Periodic solutions of some nonlinear autonomous functional differential equations, Ann. Mat. Pura Appl., 101 (1974), 263-306.

[6] F.E. BRowder, On a generalization of the Schauder fixed point point theorem, Duke Mathematical Journal 26 (1959), 291-303.

[7] D. SAUPE, Global bifurcation of periodic solution of some autonomous differential delay equations, Forschungschwerpunkt Dynamische Systeme, Report no 71, Univ. Bremen, July, 1982. 
[8] D. SAUPE, Accelerated PL - Continuation methods and periodic solutions of parameterized differential-delay equations, Ph.D. disseration (in German), Univ. Bremen, 1982.

[9] S.A. Chapin; R.D. Nussbaum, Asymptotic estimates for the periods of periodic solutions of a differential-delay equation, Mich. Math. J. 31 (1984), 215-229. 